\title{
Brundan-Kazhdan-Lusztig and Super Duality Conjectures
}

\author{
By
}

Shun-Jen CHENG* and Weiqiang WANG**

\begin{abstract}
We formulate a general super duality conjecture on connections between parabolic categories $\mathcal{O}$ of modules over Lie superalgebras and Lie algebras of type $A$, based on a Fock space formalism of their Kazhdan-Lusztig theories which was initiated by Brundan. We show that the Brundan-Kazhdan-Lusztig (BKL) polynomials for $\mathfrak{g l}(m \mid n)$ in our parabolic setup can be identified with the usual parabolic Kazhdan-Lusztig polynomials. We establish some special cases of the BKL conjecture on the parabolic category $\mathcal{O}$ of $\mathfrak{g l}(m \mid n)$-modules and additional results which support the BKL conjecture and super duality conjecture.
\end{abstract}

\section{Contents}

$\S 1 . \quad$ Introduction

$\S 2 . ~ B a s i c s$ of $q$-Multilinear Algebra

$\S 3$. The Parabolic Brundan-Kazhdan-Lusztig Theory for $\mathfrak{g l}(m \mid n)$

$\S 4$. Kazhdan-Lusztig Theory for $\mathfrak{g l}(m+n)$ Revisited and Super Duality

$\S 5$. Application of the Chuang-Rouquier $\mathfrak{s l}_{2}$-Categorification

$\S 6$. Some Results on Canonical Basis and Tilting Modules

$\S 7$. The Category $\mathcal{O}_{1,1 \mid n}^{+}$of $\mathfrak{g l}(2 \mid n)$-Modules

$\S 8$. The Category $\mathcal{O}_{m, 1 \mid 1}^{+}$of $\mathfrak{g l}(m+1 \mid 1)$-Modules

Communicated by M. Kashiwara. Received September 25, 2007.

2000 Mathematics Subject Classification(s): Primary 17B10; Secondary 17B37, $20 \mathrm{C08}$.

Key words: Lie algebras, Lie superalgebras, representation theory, super duality.

*Institute of Mathematics, Academia Sinica, Taipei, Taiwan 11529.

e-mail: chengsj@math.sinica.edu.tw

** Department of Mathematics, University of Virginia, Charlottesville, VA 22904, USA.

e-mail: ww9c@virginia.edu

(c) 2008 Research Institute for Mathematical Sciences, Kyoto University. All rights reserved. 
$\S 9$. The Category of $\mathfrak{g l}(2 \mid 1)$-Modules

References

\section{$\S 1$. Introduction}

\section{$\S 1.1$. The earlier work}

In 2003 Brundan [Br1] obtained a purely algebraic and conceptual solution to the problem of finding finite-dimensional irreducible characters of the complex Lie superalgebra $\mathfrak{g l}(m \mid n)$. Earlier Serganova [Se] found an algorithm for computing these irreducible characters using a mixture of algebraic and geometric technique. This problem can be traced back three decades earlier to $\mathrm{Kac}[\mathrm{K} 1, \mathrm{~K} 2]$, where initial progress was made. In the meantime, there has been a tremendous amount of work towards it with various partial results (see [Se, Br1, CWZ] for more references).

In Brundan's approach, the Hecke algebra modules and their bar-invariant basis in the standard Kazhdan-Lusztig (KL) theory [KL1, KL2, Deo] are replaced by the module $\Lambda^{m} \mathbb{V} \otimes \Lambda^{n} \mathbb{V}^{*}$ over the quantum group $\mathcal{U}_{q^{\mathfrak{s}} l_{\infty}}$ and its Lusztig-Kashiwara canonical basis/global basis, where $\mathbb{V}$ denotes the natural $\mathfrak{U}_{q} \mathfrak{s l}_{\infty}$-module. The Fock space $\Lambda^{m} \mathbb{V} \otimes \Lambda^{n} \mathbb{V}^{*}$ at $q=1$ should be regarded as the Grothendieck group of the category $\mathcal{O}_{m \mid n}^{+}$of finite-dimensional $\mathfrak{g l}(m \mid n)$ modules. Such a Fock space approach has been further applied successfully to study the finite-dimensional irreducible and tilting characters of other Lie superalgebras [Br3, CWZ2]. In [Br1], for the first time, a Kazhdan-Lusztig conjecture for the full category $\mathcal{O}$ of $\mathfrak{g l}(m \mid n)$-modules is formulated using the canonical basis theory of the module $\mathbb{V}^{\otimes m} \otimes\left(\mathbb{V}^{*}\right)^{\otimes n}$.

Subsequently in a joint work [CWZ] of the authors with Zhang, a connection between $\mathcal{O}_{m \mid n}^{+}$and the parabolic category $\mathcal{O}_{m+n}^{+}$of $\mathfrak{g l}(m+n)$-modules, associated with the maximal parabolic subalgebra $\mathfrak{p}_{m, n}$, was formulated. Roughly speaking, by developing further the Fock space formalism we showed that for a fixed $m$ the inverse $\operatorname{limits} \lim \mathcal{O}_{m \mid n}^{+}$and $\lim _{-} \mathcal{O}_{m+n}^{+}$, with respect to $n$, afford isomorphic Kazhdan-Lusztig theories, and moreover, we conjectured an equivalence of the two categories.

\section{$\S 1.2$. The conjectures}

Fix an $s$-tuple of positive integers $\mathbf{m}=\left(m_{1}, \ldots, m_{s}\right)$ with $\sum_{a} m_{a}=m$. In the present paper we formulate a parabolic version of Brundan's conjecture for 
a category $\mathcal{O}_{\mathbf{m} \mid n}^{+}$of $\mathfrak{g l}(m \mid n)$-modules with respect to a fairly general parabolic subalgebra $\mathfrak{p}_{\mathbf{m}, n}$, and a super duality conjecture on the equivalence of categories of $\lim _{\longleftarrow} \mathcal{O}_{\mathbf{m} \mid n}^{+}$and $\lim _{\longleftarrow} \mathcal{O}_{\mathbf{m}+n}^{+}$, where $\mathcal{O}_{\mathbf{m}+n}^{+}$stands for an analogous parabolic category of $\mathfrak{g l}(m+n)$-modules.

According to this version of Brundan-Kazhdan-Lusztig (BKL) conjecture, the parabolic Verma, tilting, and irreducible modules in $\mathcal{O}_{\mathbf{m} \mid n}^{+}$correspond respectively to the monomial, canonical, and dual canonical basis elements in the Fock space $\mathcal{E}^{\mathbf{m} \mid n}:=\bigotimes_{a} \Lambda^{m_{a}} \mathbb{V} \bigotimes \Lambda^{n} \mathbb{V}^{*}$ (or rather in a suitable topological completion). On the other hand, one has an increasingly better known reformulation of the Kazhdan-Lusztig conjecture (theorem of Beilinson-Bernstein [BB] and Brylinski-Kashiwara [BK]; see Soergel [So2] for tilting module characters) that the tilting and irreducible modules in $\mathcal{O}_{\mathbf{m}+n}^{+}$correspond to the canonical and dual canonical basis elements in the Fock space $\mathcal{E}^{\mathbf{m}+n}:=\bigotimes_{a} \Lambda^{m_{a}} \mathbb{V} \bigotimes \Lambda^{n} \mathbb{V}$. Alternatively, the KL conjecture can also be viewed as a special case of the parabolic BKL conjecture with $n=0$.

Even though the formulation of the above conjectures in such a parabolic generality seems inevitable or unsurprising to some experts after the works [Br1] and [CWZ], we hope that the general reader may still find it worthwhile and helpful, as it clarifies the scope and the limitation of these new developments. Sometimes a more general conjecture has a better chance for (partial) verification as they involve simpler combinatorics (compare the treatment of parabolic KL polynomials by Deodhar [Deo] and its impact on the related development of parabolic KL conjectures).

\section{$\S 1.3$. The main results}

We establish various compatibility results on the bar involution, canonical and dual canonical bases of the Fock spaces $\mathcal{E}^{\mathbf{m} \mid n}$ and $\mathcal{E}^{\mathbf{m}+n}$, when $n$ varies. In particular there is a canonical isomorphism of these spaces at the limit $n \rightarrow \infty$. This allows us to identify Brundan's KL polynomials with the classical type $A$ parabolic KL polynomials. We show that the canonical basis elements in $\mathcal{E}^{\mathbf{m} \mid n}$, and then in $\mathcal{E}^{\mathbf{m}+n}$, stabilize in a suitable sense for $n \gg 0$. We further establish in Theorem 4.8 a positivity result on the expansion of the divided powers of Chevalley generators acting on (dual) canonical basis elements, confirming a parabolic version of [Br1, Conjecture 2.28]. As a corollary it follows that every canonical basis element in the Fock space $\mathcal{E}^{\mathbf{m} \mid n}$ is a finite sum of monomials.

In an approach different from [CWZ], we establish in Section 3 properties of tilting modules in $\mathcal{O}_{\mathbf{m} \mid n}^{+}$for varying $n$ without assuming either the validity of the BKL conjecture or using explicit formulas of canonical basis. We introduce 
truncation functors that interpolate the categories $\mathcal{O}_{\mathbf{m} \mid n}^{+}$and $\mathcal{O}_{\mathbf{m}+n}^{+}$for varying $n$, and establish various compatibility results. In particular, we prove a stability result for the tilting modules $U_{n}(\lambda)$ in $\mathcal{O}_{\mathbf{m} \mid n}^{+}$for a given weight $\lambda$, i.e., the $U_{n}(\lambda)$ have the same finite Verma flag structures for every $n \gg 0$ (where it is understood that a tail of zeros is added to $\lambda$ for larger $n$ ). The connections between canonical bases in various Fock spaces $\mathcal{E}^{\mathbf{m} \mid n}$ and $\mathcal{E}^{\mathbf{m}+n}$ further allow us to establish the same stability result for tilting modules in $\mathcal{O}_{\mathbf{m}+n}^{+}$. (We are not aware of any other proofs even though such a statement appears to be classical).

The parabolic BKL conjecture for $\mathcal{O}_{\mathbf{m} \mid n}^{+}$would follow from the properties of the truncation maps and functors established in this paper, under the assumption of the validity of the super duality conjecture. Also, it would follow from the validity of Brundan's conjecture on the full category $\mathcal{O}$. However, the parabolic formulation of this paper can still be useful, since most of our results stated above either do not make sense or cannot be proved for now in the setup of the full category $\mathcal{O}$ or its associated Fock space.

Note that the known proofs of the classical Kazhdan-Lusztig conjectures ultimately rely on geometric machinery. For lack of such geometric tools, the BKL conjecture, or the super duality conjecture in general, presently appears to lie beyond our reach. We obtain some partial verification of the BKL conjecture under some "regularity" condition on the weights. In the special case when $\mathbf{m}=(1,1)$ and $n$ is arbitrary, we establish the parabolic BKL conjecture and a weak version of the super duality conjecture, where among others the method of the $\mathfrak{s l}_{2}$-categorification of Chuang-Rouquier [CR] is used. We also establish the parabolic BKL conjecture in another special case when $\mathbf{m}=(m, 1)$ and $n=1$. In both cases, we find explicit formulas for the canonical basis and thus the weights of Verma modules of Verma flags of the tilting modules. (Our approach can be adapted to give a purely algebraic proof of the usual type $A$ Kazhdan-Lusztig conjecture in the corresponding parabolic and low rank cases).

\section{§1.4. The organization}

The layout of this paper is as follows.

- In Section 2, we define the canonical and dual canonical bases for the Fock spaces $\mathcal{E}^{\mathbf{m} \mid n}$, and investigate their relationship for varying $n$ under the truncation maps.

- In Section 3, we formulate the parabolic BKL conjecture on $\mathcal{O}_{m \mid n}^{+}$and 
establish various results on tilting modules.

- In Section 4, we reformulate the classical parabolic Kazhdan-Lusztig conjecture of type $A$ by means of the Fock space $\mathcal{E}^{\mathbf{m + n}}$ and also present our general super duality conjecture. We obtain a key isomorphism result on Fock spaces which underlies the super duality conjecture.

- In Section 5, we adapt the powerful machinery of the $\mathfrak{s l}_{2}$-categorification of Chuang-Rouquier to the category $\mathcal{O}_{\mathbf{m} \mid n}^{+}$. Some formal consequences of the $\mathfrak{s l}_{2}$-categorification are used in the subsequent sections.

- In Section 6, as a preparation for the next sections, we establish several technical results regarding the tilting modules in the category $\mathcal{O}_{\mathbf{m} \mid n}^{+}$. We also give an explicit description of the tilting modules when the weights satisfy a regularity condition, which partially verifies the parabolic BKL conjecture.

- In Section 7, we establish the parabolic BKL conjecture and a weak version of the super duality conjecture when $\mathbf{m}=(1,1)$. In Section 8 , we establish the parabolic BKL conjecture for $\mathcal{O}_{m, 1 \mid 1}^{+}$.

- In Section 9 , we focus on the category $\mathcal{O}_{2 \mid 1}^{+}$of $\mathfrak{g l}(2 \mid 1)$-modules. We work out explicitly the Verma flag structures for the tilting and projective modules, as well as the composition series of Verma modules. We further classify the projective tilting modules.

We often omit the details of proofs when they are very similar or even identical to those for the special case (i.e. $\mathbf{m}=m$ ) treated in [Br1] and [CWZ] to keep the paper within a reasonable size. The reader is recommended to have copies of these two papers at hand when reading the present paper.

\section{$\S 2 . \quad$ Basics of $q$-Multilinear Algebra}

In this section we set up various notations, compatible with [CWZ] which is our special case when $\mathbf{m}=m$. We refer to [CWZ, Section 2] for more detail (also see $[\mathrm{Br} 1]$ ).

\section{$\S 2.1$. The quantum group}

The quantum group $U_{q} \mathfrak{g l}_{\infty}$ is the $\mathbb{Q}(q)$-algebra generated by $E_{a}, F_{a}, K_{a}^{ \pm 1}$, $a \in \mathbb{Z}$, subject to the relations 


$$
\begin{array}{rlr}
K_{a} K_{a}^{-1}=K_{a}^{-1} K_{a}=1, & K_{a} K_{b}=K_{b} K_{a}, \\
K_{a} E_{b} K_{a}^{-1}=q^{\delta_{a, b}-\delta_{a, b+1} E_{b},} & K_{a} F_{b} K_{a}^{-1}=q^{\delta_{a, b+1}-\delta_{a, b}} F_{b}, \\
E_{a} F_{b}-F_{b} E_{a}=\delta_{a, b}\left(K_{a, a+1}-K_{a+1, a}\right) /\left(q-q^{-1}\right), & \\
E_{a} E_{b}=E_{b} E_{a}, & F_{a} F_{b}=F_{b} F_{a}, \quad \text { if }|a-b|>1, \\
E_{a}^{2} E_{b}+E_{b} E_{a}^{2}=\left(q+q^{-1}\right) E_{a} E_{b} E_{a}, & \text { if }|a-b|=1, \\
F_{a}^{2} F_{b}+F_{b} F_{a}^{2}= & \left(q+q^{-1}\right) F_{a} F_{b} F_{a}, & \text { if }|a-b|=1 .
\end{array}
$$

Here and below $K_{a, b}:=K_{a} K_{b}^{-1}$ for $a \neq b \in \mathbb{Z}$. Define the bar involution on $U_{q} \mathfrak{g l}_{\infty}$ to be the anti-linear automorphism ${ }^{-}: E_{a} \mapsto E_{a}, \quad F_{a} \mapsto F_{a}, \quad K_{a} \mapsto$ $K_{a}^{-1}$. Here by anti-linear we mean with respect to the automorphism of $\mathbb{Q}(q)$ given by $q \mapsto q^{-1}$.

Let $\mathbb{V}$ be the natural $U_{q} \mathfrak{g l}_{\infty}$-module with basis $\left\{v_{a}\right\}_{a \in \mathbb{Z}}$ and $\mathbb{W}:=\mathbb{V}^{*}$ the dual module with basis $\left\{w_{a}\right\}_{a \in \mathbb{Z}}$ such that $w_{a}\left(v_{b}\right)=(-q)^{-a} \delta_{a, b}$. We have

$$
\begin{array}{r}
K_{a} v_{b}=q^{\delta_{a b}} v_{b}, \quad E_{a} v_{b}=\delta_{a+1, b} v_{a}, \quad F_{a} v_{b}=\delta_{a, b} v_{a+1}, \\
K_{a} w_{b}=q^{-\delta_{a b}} w_{b}, \quad E_{a} w_{b}=\delta_{a, b} w_{a+1}, \quad F_{a} w_{b}=\delta_{a+1, b} w_{a} .
\end{array}
$$

As in [Br1, CWZ] we shall use the comultiplication $\Delta$ on $U_{q} \mathfrak{g l}_{\infty}$ defined by:

$$
\begin{aligned}
& \Delta\left(E_{a}\right)=1 \otimes E_{a}+E_{a} \otimes K_{a+1, a}, \\
& \Delta\left(F_{a}\right)=F_{a} \otimes 1+K_{a, a+1} \otimes F_{a}, \quad \Delta\left(K_{a}\right)=K_{a} \otimes K_{a} .
\end{aligned}
$$

We let $\mathcal{U}=U_{q} \mathfrak{s l}_{\infty}$ denote the subalgebra with generators $E_{a}, F_{a}, K_{a, a+1}, a \in \mathbb{Z}$.

For $k \geq 0$, set $[k]=\frac{q^{k}-q^{-k}}{q-q^{-1}}$ and $[k] !=[k][k-1] \cdots[1]$, and introduce the divided power $E_{a}^{(k)}=E_{a}^{k} /[k] !, F_{a}^{(k)}=F_{a}^{k} /[k]$ !. One has the following comultiplication formula

$$
\Delta\left(F_{a}^{(k)}\right)=\sum_{i=0}^{k} q^{i(k-i)} K_{a, a+1}^{i} F_{a}^{(k-i)} \otimes F_{a}^{(i)} .
$$

\section{$\S 2.2$. The Fock space $\mathcal{E}^{\mathbf{m}+n}$}

For $m \in \mathbb{N}, n \in \mathbb{N} \cup \infty$, we let

$$
I(m \mid n):=\{-m,-m+1, \ldots,-1\} \cup\{1,2, \ldots, n\} .
$$

Given an $s$-tuple of positive integers

$$
\mathbf{m}=\left(m_{1}, \ldots, m_{s}\right), \quad \text { where } m_{1}+\cdots+m_{s}=m,
$$


we denote by $S_{m+n}$ the symmetric group of (finite) permutations on $I(m \mid n)$, by $S_{\mathbf{m} \mid n}$ its Young subgroup $S_{m_{1}} \times \cdots S_{m_{s}} \times S_{n}$, and by $w_{0}$ the longest element in $S_{\mathbf{m} \mid n}$ for $n$ finite. Denote by $\tau_{i j}$ the transposition interchanging $i$ and $j$.

For $n \in \mathbb{N} \cup \infty$, we let $\mathbb{Z}^{m+n}$ or $\mathbb{Z}^{m \mid n}$ be the set of integer-valued functions on $I(m \mid n)$. Set (for a finite $n$ )

$$
\begin{aligned}
\mathbb{Z}_{+}^{\mathbf{m}+n}:= & \left\{f \in \mathbb{Z}^{m+n} \mid f(-m)>\cdots>f\left(-m+m_{1}-1\right),\right. \\
& f\left(-m+m_{1}\right)>\cdots>f\left(-m+m_{1}+m_{2}-1\right), \\
& \left.\ldots, f\left(-m_{s}\right)>\cdots>f(-1), f(1)>\cdots>f(n)\right\}, \\
\mathbb{Z}_{++}^{\mathbf{m}+n}:= & \left\{f \in \mathbb{Z}_{+}^{\mathbf{m}+n} \mid f(n) \geq 1-n\right\} . \\
\mathbb{Z}_{+}^{\mathbf{m}+\infty}:= & \left\{f \in \mathbb{Z}^{m+\infty} \mid f(-m)>\cdots>f\left(-m+m_{1}-1\right),\right. \\
& f\left(-m+m_{1}\right)>\cdots>f\left(-m+m_{1}+m_{2}-1\right), \ldots, \\
& \left.f\left(-m_{s}\right)>\cdots>f(-1), f(1)>f(2)>\cdots ; f(i)=1-i \text { for } i \gg 0\right\} .
\end{aligned}
$$

Occasionally, we shall denote $\mathbb{Z}_{++}^{\mathbf{m}+\infty} \equiv \mathbb{Z}_{+}^{\mathbf{m}+\infty}$.

For $n \in \mathbb{N}$, one can define a right action of the Hecke algebra $\mathcal{H}_{n}$ of type $A$ on the tensor space $\mathbb{V}^{\otimes n}$ which commutes with the action via the $(n-1) s t$ iterated comultiplication $\Delta^{n-1}$ of $\mathcal{U}$ following Jimbo [Jim]. One can define the space $\Lambda^{n} \mathbb{V}$ of finite $q$-wedges as a quotient space of $\mathbb{V}^{\otimes n}$ via the skew $q$ symmetrizer from $\mathcal{H}_{n}$ and then the space $\Lambda^{\infty} \mathbb{V}$ of infinite-wedges by taking the limit $n \rightarrow \infty$ appropriately as done in [KMS]. These spaces are naturally U-modules. The q-wedge $v_{a_{1}} \wedge \cdots \wedge v_{a_{n}}$ is an element of $\Lambda^{n} \mathbb{V}$, which is the image of $v_{a_{1}} \otimes \cdots \otimes v_{a_{n}}$ under the canonical map when $\Lambda^{n} \mathbb{V}$ is regarded as a quotient of $\mathbb{V}^{\otimes n}$. The elements $v_{a_{1}} \wedge \cdots \wedge v_{a_{n}}$, for $a_{1}>\cdots>a_{n}$ and $a_{i} \in \mathbb{Z}$, form a basis for $\Lambda^{n} \mathbb{V}$. Similarly, the $\mathcal{U}$-module $\Lambda^{\infty} \mathbb{V}$ has a basis given by the infinite $q$-wedges $v_{m_{1}} \wedge v_{m_{2}} \wedge v_{m_{3}} \wedge \cdots$, where $m_{1}>m_{2}>m_{3}>\cdots$, and $m_{i}=1-i$ for $i \gg 0$ (our $\Lambda^{\infty} \mathbb{V}$ is $F_{(0)}$ in [KMS]). Alternatively, $\Lambda^{\infty} \mathbb{V}$ has a basis

$$
|\lambda\rangle:=v_{\lambda_{1}} \wedge v_{\lambda_{2}-1} \wedge v_{\lambda_{3}-2} \wedge \cdots,
$$

where $\lambda=\left(\lambda_{1}, \lambda_{2}, \ldots\right)$ runs over the set of all partitions.

For $n \in \mathbb{N} \cup \infty$, the space

$$
\varepsilon^{\mathbf{m}+n}:=\bigotimes_{a=1}^{s} \Lambda^{m_{a}} \mathbb{V} \bigotimes \Lambda^{n} \mathbb{V}
$$

is acted upon by $\mathcal{U}$ via the $s$-th iterated comultiplication $\Delta^{s}$. It has the monomial basis

$$
\mathcal{K}_{f}:=v_{f\left[-m,-m+m_{1}\right)} \otimes v_{f\left[-m+m_{1},-m+m_{1}+m_{2}\right)} \otimes \cdots \otimes v_{f\left[-m_{s},-1\right]} \otimes v_{f[1, n]},
$$


where $f$ runs over the set $\mathbb{Z}_{+}^{\mathbf{m}+n}$ and we have denoted by, for given $a \leq b$,

$$
v_{f[a, b]} \equiv v_{f[a, b+1)}:=v_{f(a)} \wedge v_{f(a+1)} \wedge \cdots \wedge v_{f(b)} .
$$

The Bruhat ordering $\leq$ on $\mathbb{Z}^{m+n}$, which comes from the Bruhat ordering on $S_{m+n}$, is the transitive closure of the relation $f<f \cdot \tau_{i j}$, if $f(i)<f(j)$, for $i, j \in I(m \mid n)$ with $i<j$. This induces the Bruhat ordering $\leq$ on $\mathbb{Z}_{+}^{\mathbf{m}+n}$.

Let $P$ be the free abelian group with basis $\left\{\epsilon_{a} \mid a \in \mathbb{Z}\right\}$ equipped with a bilinear form $(\cdot \mid \cdot)$, for which the $\epsilon_{a}$ 's are orthonormal. For later use, we define the $\epsilon$-weights on $\mathbb{Z}^{m+n}$ :

$$
\mathrm{wt}^{\epsilon}(f):=\sum_{i \in I(m \mid n)} \epsilon_{f(i)}, \quad \text { for } f \in \mathbb{Z}^{m+n} .
$$

\section{$\S 2.3$. The Fock space $\mathcal{E}^{\mathbf{m} \mid n}$}

Set (for a finite $n$ )

$$
\begin{aligned}
& \mathbb{Z}_{+}^{\mathbf{m} \mid n}:=\{f \in \mathbb{Z}^{m \mid n} \mid f(-m)>\cdots>f\left(-m+m_{1}-1\right), \\
& f\left(-m+m_{1}\right)>\cdots>f\left(-m+m_{1}+m_{2}-1\right), \\
&\left.\ldots, f\left(-m_{s}\right)>\cdots>f(-1), f(1)<\cdots<f(n)\right\}, \\
& \mathbb{Z}_{++}^{\mathbf{m} \mid n}:=\{f\left.\mathbb{Z}_{+}^{\mathbf{m} \mid n} \mid f(n) \leq n\right\} . \\
& \mathbb{Z}_{+}^{\mathbf{m} \mid \infty}:=\left\{f \in \mathbb{Z}^{m+\infty} \mid f(-m)>\cdots>f\left(-m+m_{1}-1\right),\right. \\
& f\left(-m+m_{1}\right)>\cdots>f\left(-m+m_{1}+m_{2}-1\right), \ldots, \\
&\left.\quad f\left(-m_{s}\right)>\cdots>f(-1), f(1)<f(2)<\cdots ; f(i)=i \text { for } i \gg 0\right\} .
\end{aligned}
$$

(Occasionally, we also denote $\mathbb{Z}_{++}^{\mathbf{m} \mid \infty} \equiv \mathbb{Z}_{+}^{\mathbf{m} \mid \infty}$.)

Recall that $\mathbb{W}=\mathbb{V}^{*}$ is the $\mathcal{U}$-module dual to $V$ with basis $\left\{w_{a}\right\}_{a \in \mathbb{Z}}$. The space $\mathbb{W}^{\otimes n}$ admits a right action of the Hecke algebra $\mathcal{H}_{n}$ which commutes with the action via $\Delta^{n-1}$ of the quantum group $\mathcal{U}$. In the same way using the skew $q$-symmetrizer, the $\mathcal{U}$-module $\Lambda^{n} \mathbb{W}$ has a basis given by $w_{a_{1}} \wedge w_{a_{2}} \wedge \cdots \wedge w_{a_{n}}$ for $a_{1}<\ldots<a_{n}$. Similarly, we construct the space $\Lambda^{\infty} \mathbb{W}$ of semi-infinite $q$-wedges $w_{n_{1}} \wedge w_{n_{2}} \wedge \cdots$, where $n_{i}=i$ for $i \gg 0$, which carries a $\mathcal{U}$-module structure. Writing the conjugate partition of $\lambda$ as $\lambda^{\prime}=\left(\lambda_{1}^{\prime}, \lambda_{2}^{\prime}, \ldots\right)$, we set

$$
\left|\lambda_{*}^{\prime}\right\rangle:=w_{1-\lambda_{1}^{\prime}} \wedge w_{2-\lambda_{2}^{\prime}} \wedge w_{3-\lambda_{3}^{\prime}} \wedge \cdots .
$$

The set $\left\{\left|\lambda_{*}^{\prime}\right\rangle\right\}$ is a basis for $\Lambda^{\infty} \mathbb{W}$. 
For $n \in \mathbb{N} \cup \infty$, we denote

$$
\mathcal{E}^{\mathbf{m} \mid n}=\bigotimes_{a=1}^{s} \Lambda^{m_{a}} \mathbb{V} \bigotimes \Lambda^{n} \mathbb{W}
$$

which is acted upon by $\mathcal{U}$ via the $s$-th iterated comultiplication $\Delta^{s}$. The space $\mathcal{E}^{\mathbf{m} \mid n}$ has the monomial basis

$$
K_{f}:=v_{f\left[-m,-m+m_{1}\right)} \otimes v_{f\left[-m+m_{1},-m+m_{1}+m_{2}\right)} \otimes \cdots \otimes v_{f\left[-m_{s},-1\right]} \otimes w_{f[1, n]},
$$

where $f$ runs over $\mathbb{Z}_{+}^{\mathbf{m} \mid n}$ and $w_{f[1, n]}=w_{f(1)} \wedge \cdots \wedge w_{f(n)}$.

For $i \in I(m \mid n)$ we define $d_{i} \in \mathbb{Z}^{m \mid n}$ by $j \mapsto-\operatorname{sgn}(i) \delta_{i j}$. For $f, g \in \mathbb{Z}^{m \mid n}$, we write $f \downarrow g$ if one of the following holds:

(1) $g=f-d_{i}+d_{j}$ for some $i<0<j$ such that $f(i)=f(j)$;

(2) $g=f \cdot \tau_{i j}$ for some $i<j<0$ such that $f(i)>f(j)$;

(3) $g=f \cdot \tau_{i j}$ for some $0<i<j$ such that $f(i)<f(j)$.

The super Bruhat ordering on $\mathbb{Z}^{m \mid n}$ is defined as follows: for $f, g \in \mathbb{Z}^{m \mid n}$, we say that $f \succcurlyeq g$, if there exists a sequence $f=h_{1}, \ldots, h_{r}=g \in \mathbb{Z}^{m \mid n}$ such that $h_{1} \downarrow h_{2} \cdots h_{r-1} \downarrow h_{r}$. It can also be described cf. [Br1, $\left.\S 2 \mathrm{~b}\right]$ by a number of inequalities in terms of the $\epsilon$-weights on $\mathbb{Z}^{m \mid n}$, which are defined by:

$$
\mathrm{wt}^{\epsilon}(f):=\sum_{i \in I(m \mid n)}-\operatorname{sgn}(i) \epsilon_{f(i)}, \quad \text { for } f \in \mathbb{Z}^{m \mid n} .
$$

The super Bruhat ordering on $\mathbb{Z}^{m \mid n}$ induces a super Bruhat ordering on the subsets $\mathbb{Z}_{+}^{\mathbf{m} \mid n}, \mathbb{Z}_{++}^{\mathbf{m} \mid n}$, and $\mathbb{Z}_{+}^{\mathbf{m} \mid \infty}$.

For $n \in \mathbb{N}$, the degree of atypicality (or atypicality number) of $f \in \mathbb{Z}^{m \mid n}$ is defined to be

$$
\# f:=\frac{1}{2}\left(m+n-\sum_{a \in \mathbb{Z}}\left|\left(\mathrm{wt}^{\epsilon}(f), \epsilon_{a}\right)\right|\right) .
$$

For $f \in \mathbb{Z}^{m \mid \infty}$, we define $\# f$ to be the degree of atypicality of the restriction of $f$ to $I(m \mid n)$ for $n \gg 0$ (which is clearly well-defined).

If $f, g \in \mathbb{Z}_{+}^{\mathbf{m} \mid n}$ are comparable under the super Bruhat ordering, then $\# f=\# g$. If $\# f=0$, we say that $f$ is typical; otherwise $f$ is atypical. An element $\mathbb{Z}_{+}^{\mathbf{m} \mid n}$ is minimal in the super Bruhat ordering if and only if $f$ is typical and $f \cdot \tau_{i j}$ is not conjugate under the action of $S_{\mathbf{m} \mid n}$ to an element in $\mathbb{Z}_{+}^{\mathbf{m} \mid n}$ whenever $f(i)>f(j)$ with $i<j<0$. 


\section{§2.4. Bases for $\widehat{\mathcal{E}}^{\mathbf{m} \mid n}$}

Let $n \in \mathbb{N} \cup \infty$. For $d \in \mathbb{N}$ let $\mathcal{E}_{>-d}^{\mathbf{m} \mid n}$ be the $\mathbb{Q}(q)$-subspace of $\mathcal{E}^{\mathbf{m} \mid n}$ spanned by $K_{f}$ with $f(i) \geq-d$, for all $i \in\{1, \cdots, n\}$. Following [Br1, CWZ] we shall denote a certain topological completion of $\mathcal{E}^{\mathbf{m} \mid n}$ by $\widehat{\mathcal{E}}^{\mathbf{m} \mid n}$ whose elements may be viewed as infinite $\mathbb{Q}(q)$-linear combinations of elements in $\mathcal{E}^{\mathbf{m} \mid n}$, which under the projection onto $\mathcal{E}_{\geq-d}^{\mathbf{m} \mid n}$ are finite sums for all $d \in \mathbb{N}$ (cf. [Br1, $\left.\S 2-\mathrm{d}\right]$ ).

We can define a quasi-matrix following [Lu2, Chap. 24, 27], that extends the bar-involutions on $\mathcal{E}^{\mathbf{m} \mid 0}$ and on $\mathcal{E}^{0 \mid n}$. Using this we can then construct a bar-involution on $\mathcal{E}^{\mathbf{m} \mid n}$. The following proposition is a variant of [Br1, Theorem 2.14, Theorem 3.5] and results of Lusztig, and it can be proved similarly.

Proposition 2.1. Let $n \in \mathbb{N} \cup \infty$. There exists a unique continuous, anti-linear bar map ${ }^{-}: \widehat{\mathcal{\varepsilon}}^{\mathbf{m} \mid n} \rightarrow \widehat{\mathcal{E}}^{\mathbf{m} \mid n}$ such that

(1) $\overline{K_{f}}=K_{f}$, for all $f \in \mathbb{Z}_{+}^{\mathbf{m} \mid n}$ minimal in the super Bruhat ordering.

(2) $\overline{X u}=\bar{X} \bar{u}$, for all $X \in \mathcal{U}$ and $u \in \widehat{\mathcal{E}}^{\mathbf{m} \mid n}$.

(3) The bar map is an involution.

(4) $\overline{K_{f}}=K_{f}+(*)$, where $(*)$ is a (possibly infinite) $\mathbb{Z}\left[q, q^{-1}\right]$-linear combination of $K_{g}$ 's, with $g \in \mathbb{Z}_{+}^{\mathbf{m} \mid n}$ such that $g \prec f$.

The next theorem now follows by standard arguments (cf. [KL1, Lu2, $\mathrm{Br} 1])$.

Theorem 2.2. Let $n \in \mathbb{N} \cup \infty$. There exist unique canonical basis $\left\{U_{f}\right\}$ and dual canonical basis $\left\{L_{f}\right\}$, where $f \in \mathbb{Z}_{+}^{\mathbf{m} \mid n}$, for $\widehat{\mathcal{E}}^{\mathbf{m} \mid n}$ such that

(1) $\bar{U}_{f}=U_{f}$ and $\bar{L}_{f}=L_{f}$.

(2) $U_{f} \in K_{f}+\widehat{\sum}_{g \in \mathbb{Z}_{+}^{\mathrm{m} \mid n}} q \mathbb{Z}[q] K_{g}$ and $L_{f} \in K_{f}+\widehat{\sum}_{g \in \mathbb{Z}_{+}^{\mathrm{m} \mid n}} q^{-1} \mathbb{Z}\left[q^{-1}\right] K_{g}$.

(3) $U_{f}=K_{f}+(*)$ and $L_{f}=K_{f}+(* *)$, where $(*)$ and $(* *)$ are (possibly infinite) $\mathbb{Z}\left[q, q^{-1}\right]$-linear combinations of $K_{g}$ 's, with $g \in \mathbb{Z}_{+}^{\mathbf{m} \mid n}$ such that $g \prec f$.

The $\widehat{\sum}$ here and further denotes a possibly infinite sum.

Let $n \in \mathbb{N} \cup \infty$. Generalizing [Br1], we define the Brundan-KazhdanLusztig polynomials $u_{g, f}(q) \in \mathbb{Z}[q], \ell_{g, f}(q) \in \mathbb{Z}\left[q^{-1}\right]$ associated to $f, g \in \mathbb{Z}_{+}^{\mathbf{m} \mid n}$ by

$$
U_{f}=\sum_{g \in \mathbb{Z}_{+}^{\mathbf{m} \mid n}} u_{g, f}(q) K_{g}, \quad L_{f}=\sum_{g \in \mathbb{Z}_{+}^{\mathbf{m} \mid n}} \ell_{g, f}(q) K_{g} .
$$


Note that $u_{g, f}(q)=\ell_{g, f}(q)=0$ unless $g \preccurlyeq f, u_{f, f}(q)=\ell_{f, f}(q)=1$, and $u_{g, f}(q) \in q \mathbb{Z}[q], \ell_{g, f}(q) \in q^{-1} \mathbb{Z}\left[q^{-1}\right]$ for $g \neq f$.

Remark 2.3. By studying a certain symmetric bilinear form on $\widehat{\mathcal{E}}^{\mathbf{m} \mid n}$ such that $\left\langle L_{f}, U_{-g \cdot w_{0}}\right\rangle=\delta_{f, g}$ for all $f, g \in \mathbb{Z}_{+}^{\mathbf{m} \mid n}$, one can show (as in [Br1, 2 -i,3-c] for the special cases for $\mathbf{m}=(1, \ldots, 1)$ or $\mathbf{m}=m)$ that

$$
K_{f}=\sum_{g \in \mathbb{Z}_{+}^{\mathbf{m} \mid n}} u_{-g \cdot w_{0},-f \cdot w_{0}}\left(q^{-1}\right) L_{g}=\sum_{g \in \mathbb{Z}_{+}^{\mathbf{m} \mid n}} \ell_{-g \cdot w_{0},-f \cdot w_{0}}\left(q^{-1}\right) U_{g}, \quad f \in \mathbb{Z}_{+}^{\mathbf{m} \mid n} .
$$

Remark 2.4. Let $\mathbf{n}=\left(n_{1}, \ldots, n_{r}\right) \in \mathbb{N}^{r}$ for $r \geq 1$. One can generalize readily the bar-involution, the monomial and (dual) canonical bases to the more general space $\mathcal{E}^{\mathbf{m} \mid \mathbf{n}}:=\otimes_{a=1}^{s} \Lambda^{m_{a}} \mathbb{V} \otimes \otimes_{b=1}^{r} \Lambda^{n_{b}} \mathbb{W}$. The bases are naturally parameterized by a set denoted by $\mathbb{Z}_{+}^{\mathbf{m} \mid \mathbf{n}}$, which is an obvious generalization of $\mathbb{Z}_{+}^{\mathbf{m} \mid n}$.

\section{§2.5. The truncation map}

Let $n$ be finite. Denote by $\mathcal{E}_{+}^{\mathbf{m} \mid n}$ the subspace of $\mathcal{E}^{\mathbf{m} \mid n}$ spanned by $K_{f}$, for $f \in \mathbb{Z}_{++}^{\mathbf{m} \mid n}$. For $\infty \geq n^{\prime}>n$, and $f \in \mathbb{Z}_{+}^{\mathbf{m} \mid n^{\prime}}$ (respectively $f \in \mathbb{Z}_{+}^{\mathbf{m}+n^{\prime}}$ ), we define $f^{(n)} \in \mathbb{Z}_{+}^{\mathbf{m} \mid n}$ (respectively $f^{(n)} \in \mathbb{Z}_{+}^{\mathbf{m}+n}$ ) to be the restriction of $f$ to $I(m \mid n)$. We define the truncation map to be the $\mathbb{Q}(q)$-linear map

$$
\mathfrak{T r}_{n^{\prime}, n}: \widehat{\mathcal{E}}_{+}^{\mathbf{m} \mid n^{\prime}} \longrightarrow \widehat{\mathcal{E}}_{+}^{\mathbf{m} \mid n},
$$

which sends $K_{f}$ to $K_{f(n)}$ if $f(i)=i$, for all $i \geq n+1$, and to 0 otherwise. We will write $\mathfrak{T r}_{n^{\prime}, n}$ as $\mathfrak{T r}_{n}$ when no ambiguity arises.

Proposition 2.5. For $\infty \geq n^{\prime}>n$, the truncation map $\mathfrak{T r}_{n^{\prime}, n}: \widehat{\mathcal{E}}_{+}^{\mathbf{m} \mid n^{\prime}}$ $\rightarrow \widehat{\mathcal{E}}_{+}^{\mathbf{m} \mid n}$ commutes with the bar-involution.

Proof. It suffices to prove the case $n^{\prime}=n+1$. The proof of [CWZ, Proposition 2.8] for the special case when $\mathbf{m}=m$ using the quasi $R$-matrix carries over to this general situation.

Corollary 2.6. Let $\infty \geq n^{\prime}>n$.

(1) $\left\{U_{f}\right\}_{f \in \mathbb{Z}_{++}^{\mathrm{m} \mid n}}$ (respectively $\left\{L_{f}\right\}_{f \in \mathbb{Z}_{++}^{\mathrm{m} \mid n}}$ is a basis for $\widehat{\mathcal{E}}_{+}^{\mathbf{m} \mid n}$.

(2) $\mathfrak{T r}_{n^{\prime}, n}$ sends $U_{f} \in \widehat{\mathcal{E}}_{+}^{\mathbf{m} \mid n^{\prime}}$ to $U_{f^{(n)}}$ if $f(i)=i$ for all $i \geq n+1$, and to 0 otherwise. 
(3) $\mathfrak{T r}_{n^{\prime}, n}$ sends $L_{f} \in \widehat{\mathcal{E}}_{+}^{\mathbf{m} \mid n^{\prime}}$ to $L_{f(n)}$ if $f(i)=i$ for all $i \geq n+1$, and to 0 otherwise.

(4) For $f, g \in \mathbb{Z}_{++}^{\mathbf{m} \mid n^{\prime}}$ such that $f(i)=g(i)=i$ for all $i \geq n+1$, we have

$$
u_{g, f}(q)=u_{g^{(n)}, f^{(n)}}(q), \quad \ell_{g, f}(q)=\ell_{g^{(n)}, f^{(n)}}(q) .
$$

\section{§3. The Parabolic Brundan-Kazhdan-Lusztig Theory for $\mathfrak{g l}(m \mid n)$}

\section{$\S 3.1$. The category $\mathcal{O}_{\mathbf{m} \mid n}^{+}$}

For $m, n \in \mathbb{N}$ the Lie superalgebra $\mathfrak{g}=\mathfrak{g l}(m \mid n)$ is generated by the elementary matrices $e_{i j}$, where $i, j \in I(m \mid n)$. For $i \in I(m \mid n)$, let $\bar{i}=\overline{0}$ if $i<0$ and $\bar{i}=\overline{1}$ if $i>0$. Let $\mathfrak{h}$ be the standard Cartan subalgebra of $\mathfrak{g}$ consisting of the diagonal matrices, $\mathfrak{b}$ the standard Borel subalgebra of the upper triangular matrices, and $\Delta^{+}$the set of positive roots for $\mathfrak{g}$. By means of the natural inclusion $\mathfrak{g l}(m \mid n) \subseteq \mathfrak{g l}(m \mid n+1)$ via $I(m \mid n) \subseteq I(m \mid n+1)$, we let $\mathfrak{g l}(m \mid \infty):=\underset{\vec{n}}{\lim } \mathfrak{g l}(m \mid n)$.

Recall that $\mathbf{m}=\left(m_{1}, \ldots, m_{s}\right)$ with $\sum_{i} m_{i}=m$. Consider the Levi subalgebra $\mathfrak{l}:=\mathfrak{g l}\left(m_{1}\right) \oplus \cdots \oplus \mathfrak{g l}\left(m_{s}\right) \oplus \mathfrak{g l}(n)$ and the corresponding parabolic subalgebra $\mathfrak{p}:=\mathfrak{l}+\mathfrak{b}$ of $\mathfrak{g}$. (We shall occasionally write $\mathfrak{p}_{n}$ if we need to keep track of $n$.)

Let $\left\{\delta_{i} \mid i \in I(m \mid n)\right\}$ be the basis of $\mathfrak{h}^{*}$ dual to $\left\{e_{i i} \mid i \in I(m \mid n)\right\}$. Let $X_{m \mid n}$ be the set of integral weights $\lambda=\sum_{i \in I(m \mid n)} \lambda_{i} \delta_{i}, \lambda_{i} \in \mathbb{Z}$. A symmetric bilinear form on $\mathfrak{h}^{*}$ is defined by

$$
\left(\delta_{i} \mid \delta_{j}\right)=-\operatorname{sgn}(i) \delta_{i j}, \quad i, j \in I(m \mid n) .
$$

Define

$$
\begin{gathered}
X_{\mathbf{m} \mid n}^{+}:=\left\{\lambda \in X_{m \mid n} \mid \lambda_{-m} \geq \cdots \geq \lambda_{-m+m_{1}-1},\right. \\
\lambda_{-m+m_{1}} \geq \cdots \geq \lambda_{-m+m_{1}+m_{2}-1}, \\
\left.\cdots, \lambda_{-m_{s}} \geq \cdots \geq \lambda_{-1}, \lambda_{1} \geq \cdots \geq \lambda_{n}\right\}, \\
X_{\mathbf{m} \mid n}^{++}:=\left\{\lambda \in X_{\mathbf{m} \mid n}^{+} \mid \lambda_{n} \geq 0\right\} .
\end{gathered}
$$

We may regard an element $\lambda$ in $X_{\mathbf{m} \mid n}^{++}$as an element in $X_{\mathbf{m} \mid n^{\prime}}^{++}$for $n^{\prime}>n$ by adjoining zeros, i.e. letting $\lambda_{i}=0$ for $n^{\prime} \geq i \geq n+1$. Let

$$
X_{\mathbf{m} \mid \infty}^{++} \equiv X_{\mathbf{m} \mid \infty}^{+}:=\lim _{\vec{n}} X_{\mathbf{m} \mid n}^{++} \text {. }
$$


For $n \in \mathbb{N} \cup \infty$ define

$$
\rho=-\sum_{i \in I(m \mid n)} i \delta_{i}
$$

Define a bijection

$$
X_{m \mid n} \longrightarrow \mathbb{Z}^{m \mid n}, \quad \lambda \mapsto f_{\lambda},
$$

where $f_{\lambda} \in \mathbb{Z}^{m \mid n}$ is given by $f_{\lambda}(i)=\left(\lambda+\rho \mid \delta_{i}\right)$ for all $i \in I(m \mid n)$. This map induces bijections $X_{\mathbf{m} \mid n}^{+} \rightarrow \mathbb{Z}_{+}^{\mathbf{m} \mid n}$ and $X_{\mathbf{m} \mid n}^{++} \rightarrow \mathbb{Z}_{++}^{\mathbf{m} \mid n}$. Using this bijection we define the notions such as the degree of atypicality, $\epsilon$-weight, partial order $\preccurlyeq$, et cetera, for elements in $X_{\mathbf{m} \mid n}^{+}$by requiring them to be compatible with those defined for elements in $\mathbb{Z}_{+}^{\mathbf{m} \mid n}$.

For $\lambda \in X_{\mathbf{m} \mid n}^{+}$, we define the parabolic Verma module to be

$$
K_{n}(\lambda):=U(\mathfrak{g}) \otimes_{U(\mathfrak{p})} L_{n}^{0}(\lambda),
$$

where $L_{n}^{0}(\lambda)$ is the irreducible $\mathfrak{l}$-module of highest weight $\lambda$ extended trivially to a $\mathfrak{p}$-module. The irreducible quotient $\mathfrak{g}$-module of $K_{n}(\lambda)$ is denoted by $L_{n}(\lambda)$. Let $\left[M: L_{n}(\lambda)\right]$ denote the multiplicity of the composition factor $L_{n}(\lambda)$ in a $\mathfrak{g l}(m \mid n)$-module $M$. When $n=\infty$ we will make it a convention to drop the subscript $n$ in $K_{n}(\lambda), L_{n}(\lambda)$ et cetera.

For $n \in \mathbb{N}, \mathcal{O}_{\mathbf{m} \mid n}^{+}$is the category of finitely generated $\mathfrak{g l}(m \mid n)$-modules $M$, with $M$ semisimple over $\mathfrak{l}$, locally finite over $\mathfrak{p}$, and

$$
M=\bigoplus_{\gamma \in X_{m \mid n}} M_{\gamma}
$$

where as usual $M_{\gamma}$ denotes the $\gamma$-weight space of $M$ with respect to $\mathfrak{h}$. Note that any object in $\mathcal{O}_{\mathbf{m} \mid n}^{+}$, when regarded as a module over its even subalgebra, has finite length by results of the classical category $\mathcal{O}$, and hence it has finite length as well. Denote by $\operatorname{Hom}_{\mathbf{m} \mid n}$ the Hom space in the category $\mathcal{O}_{\mathbf{m} \mid n}^{+}$. We twist the standard $\mathfrak{g}$-module structure on the graded dual $M^{*}$ of such an $M$ with the automorphism given by the negative supertranspose on $\mathfrak{g}$, and denote the resulting $\mathfrak{g}$-module by $M^{\tau}$. We denote by $\mathcal{O}_{\mathbf{m} \mid n}^{++}$the full subcategory of $\mathcal{O}_{\mathbf{m} \mid n}^{+}$ which consists of modules whose composition factors are of the form $L_{n}(\lambda)$ for $\lambda \in X_{\mathbf{m} \mid n}^{++}$. We let $\mathcal{O}_{\mathbf{m} \mid \infty}^{++}$be the category of finitely generated $\mathfrak{g l}(m \mid \infty)$-modules that are l-semisimple, locally finite over $\mathfrak{p}_{N} \subset \mathfrak{g l}(m \mid N)$ for all finite $N$, and such that the composition factors are of the form $L(\lambda)$ for $\lambda \in X_{\mathbf{m} \mid \infty}^{+}$. 


\section{§3.2. The truncation functor}

Let $\operatorname{wt}(v)$ denote the weight (or $\delta$-weight) of a weight vector $v$ in a $\mathfrak{g l}(m \mid n)$ module.

Definition 3.1. For $n<n^{\prime} \leq \infty$, the truncation functor $\mathfrak{t r}_{n^{\prime}, n}: \mathcal{O}_{\mathbf{m} \mid n^{\prime}}^{++}$ $\longrightarrow \mathcal{O}_{\mathbf{m} \mid n}^{++}$is the exact functor which sends an object $M$ to

$\operatorname{tr}_{n^{\prime}, n}(M):=\operatorname{span}\left\{v \in M \mid\left(\operatorname{wt}(v) \mid \delta_{k}\right)=0\right.$, for all $\left.n+1 \leq k \leq n^{\prime}\right\}$.

When $n^{\prime}$ is clear from the context we will also write $\mathfrak{t r}_{n}$ for $\mathfrak{t r}_{n^{\prime}, n}$. (It is easily checked that $\mathfrak{t r}_{n^{\prime}, n}(M) \in \mathcal{O}_{\mathbf{m} \mid n}^{++}$. $)$

We have a system of categories $\mathcal{O}_{\mathbf{m} \mid n}^{++}$with a compatible sequence of functors $\mathfrak{t r}_{n^{\prime}, n}$ in the sense that $\mathfrak{t r}_{n^{\prime \prime}, n}=\mathfrak{t r}_{n^{\prime}, n} \circ \mathfrak{t r}_{n^{\prime \prime}, n^{\prime}}$ for $n^{\prime \prime}>n^{\prime}>n$.

We have the natural inclusions $\mathfrak{g l}(m \mid n) \subset \mathfrak{g l}(m \mid n+1)$. The following is a variant of [CWZ, Lemma 3.5] and can be proved similarly.

Lemma 3.2. Let $Y=L$ or $K$. We have the natural inclusions of $\mathfrak{g l}(m \mid n)$-modules: $\quad Y_{n}(\lambda) \subseteq Y_{n+1}(\lambda)$ for $\lambda \in X_{\mathbf{m} \mid n}^{++}$. Furthermore, $\mathfrak{t r}_{n+1, n}\left(Y_{n+1}(\lambda)\right)=Y_{n}(\lambda)$.

It follows that $\cup_{n} K_{n}(\lambda)$ and $\cup_{n} L_{n}(\lambda)$ are naturally $\mathfrak{g l}(m \mid \infty)$-modules. They are direct limits of $\left\{K_{n}(\lambda)\right\}$ and $\left\{L_{n}(\lambda)\right\}$ and isomorphic to $K(\lambda)$ and $L(\lambda)$, respectively. Similarly $\cup_{n} L_{n}^{0}(\lambda) \cong L^{0}(\lambda)$ as $\mathfrak{l}$-modules.

Corollary 3.3. For $\lambda \in X_{\mathbf{m} \mid n^{\prime}}^{+}, n<n^{\prime} \leq \infty$, and $Y=L$ or $K$, we have

$$
\mathfrak{t r}_{n^{\prime}, n}\left(Y_{n^{\prime}}(\lambda)\right)=\left\{\begin{array}{rr}
Y_{n}(\lambda), & \text { if } \lambda_{i}=0 \forall i>n, \\
0, & \text { otherwise. }
\end{array}\right.
$$

Lemma 3.4. Let $\lambda \in X_{\mathbf{m} \mid n}^{++}$and $\mu \in X_{\mathbf{m} \mid n}^{+}$be such that $\mu \preccurlyeq \lambda$. Then $\mu \in X_{\mathbf{m} \mid n}^{++}$.

Proof. Recall that the super Bruhat ordering $\succcurlyeq$ is defined to be the transitive closure of the three cases of dominance $f \downarrow g$ in Subsection 2.3, where only in the first case therein the set $\{f(i)\}_{1 \leq i \leq n}$ will be changed. More precisely, one particular $f(i)$ involved in an atypical pair is replaced by some smaller integer.

Thus, thanks to $\lambda \succcurlyeq \mu,\left\{f_{\mu}(i)\right\}_{1 \leq i \leq n}$ is obtained by consecutively lowering the values $\left\{f_{\lambda}(i)\right\}_{1 \leq i \leq n}$ (which are involved in atypical pairs), whence $\mu \in$ $X_{\mathbf{m} \mid n}^{++}$. 
Given $M \in \mathcal{O}_{\mathbf{m} \mid n}^{+}$, denote by $[M]$ the corresponding element in the Grothendieck group $G\left(\mathcal{O}_{\mathbf{m} \mid n}^{+}\right)$of the category $\mathcal{O}_{\mathbf{m} \mid n}^{+}$. Corollary 3.3 and the exactness of the truncation functor $\mathfrak{t r}_{n^{\prime}, n}$ implies the following.

Proposition 3.5. For $\lambda, \mu \in X_{\mathbf{m} \mid n}^{++}$and $n^{\prime} \geq n$, we also regard $\lambda, \mu \in$ $X_{\mathbf{m} \mid n^{\prime}}^{++}$by adjoining zeros. Then, $\left[K_{n}(\lambda): L_{n}(\mu)\right]=\left[K_{n^{\prime}}(\lambda): L_{n^{\prime}}(\mu)\right]$.

Given $\lambda \in X_{\mathbf{m} \mid k}^{+}$, we denote by $\mathfrak{J}_{k}(\lambda)$ the set of the highest weights of the composition factors of $K_{k}(\lambda)$ and by $r_{k}(\lambda)$ the length of a composition series of $K_{k}(\lambda)$. Clearly, there exists $n(\lambda) \in \mathbb{N}$ such that the degree of atypicality $\# \lambda$ (where we regard $\lambda \in X_{\mathbf{m} \mid n}^{++}$by adjoining zeros) is independent of $n$ for $\infty \geq n \geq n(\lambda)$.

\section{Proposition 3.6.}

(1) The $r_{n}(\lambda)$ and $\mathfrak{J}_{n}(\lambda)$ (with the tail of zeros in a weight ignored) are independent of $n \geq n(\lambda)$. Furthermore, for $n^{\prime} \geq n \geq n(\lambda)$ the truncation functor $\mathfrak{t r}_{n^{\prime}, n}$ maps bijectively the set of Jordan-Hölder series for $K_{n^{\prime}}(\lambda)$ to the set of Jordan-Hölder series for $K_{n}(\lambda)$.

(2) The parabolic Verma module $K(\lambda)$ for $\lambda \in X_{\mathbf{m} \mid \infty}^{+}$has a finite composition series, whose composition factors are of the form $L(\mu)$ with $\mu \in X_{\mathbf{m} \mid \infty}^{+}$, and hence, $K(\lambda) \in \mathcal{O}_{\mathbf{m} \mid \infty}^{++}$. Furthermore, $[K(\lambda): L(\mu)]=\left[K_{n}(\lambda): L_{n}(\mu)\right]$.

Proof. (1) Let $n \geq n(\lambda)$. $\left[K_{n}(\lambda): L_{n}(\mu)\right] \neq 0$ for some $\mu$ implies $\mu \preccurlyeq \lambda$. Thus we have $\mu \in X_{\mathbf{m} \mid n}^{++}$and actually $\mu \in X_{\mathbf{m} \mid n(\lambda)}^{++}$by the proof of Lemma 3.4, where indeed $f_{\mu}(i)=f_{\lambda}(i)$ for $i>n(\lambda)$. Hence the first statement follows by Proposition 3.5. Now the second statement follows from the first one and Lemma 3.3 using the same argument as for [CWZ, Lemma 3.8].

(2) follows from the special case of (1) with $n^{\prime}=\infty$.

\section{$\S 3.3 . \quad$ The tilting modules}

Throughout this subsection we assume that $n$ is finite. An object $M \in$ $\mathcal{O}_{\mathbf{m} \mid n}^{+}$is said to have a Verma flag (respectively, a dual Verma flag) if it has a filtration of $\mathfrak{g l}(m \mid n)$-modules:

$$
0=M_{0} \subseteq \cdots \subseteq M_{r}=M
$$

such that each $M_{i} / M_{i-1}$ is isomorphic to a parabolic Verma module $K_{n}\left(\lambda^{i}\right)$ (respectively, $\left.K_{n}\left(\lambda^{i}\right)^{\tau}\right)$ for some $\lambda^{i} \in X_{\mathbf{m} \mid n}^{+}$. We define $\left(M: K_{n}(\mu)\right)$ for $\mu \in$ 
$X_{\mathbf{m} \mid n}^{+}$to be the number of subquotients of a Verma flag of $M$ that are isomorphic to $K_{n}(\mu)$. The tilting module associated to $\lambda \in X_{m \mid n}^{+}$in the category $\mathcal{O}_{\mathbf{m} \mid n}^{+}$is an indecomposable $\mathfrak{g l}(m \mid n)$-module $U_{n}(\lambda)$ such that $U_{n}(\lambda)$ has a Verma flag with $K_{n}(\lambda)$ at the bottom, and $\operatorname{Ext}^{1}\left(K_{n}(\mu), U_{n}(\lambda)\right)=0$ for all $\mu \in X_{\mathbf{m} \mid n}^{+}$. By a parabolic version of [Br2] as in Soergel [So2] for the usual semisimple Lie algebras, the tilting module $U_{n}(\lambda)$ in the category $\mathcal{O}_{\mathbf{m} \mid n}^{+}$exists and is unique. Following [Br2, So2], the projective cover $P_{n}(\lambda)$ of $L_{n}(\lambda)$ exists for each $\lambda \in$ $X_{\mathbf{m} \mid n}^{+}$and admits a finite Verma flag. The following is a synthesis of standard results (see [Jan, Br2]) adapted to our particular setup.

\section{Proposition 3.7.}

(1) Let $M$ be a module with a finite Verma flag and $N$ be a module with a finite dual Verma flag. Then, $\operatorname{Ext}^{i}(M, N)=0$ for all $i>0$.

(2) Let $N \in \mathcal{O}_{\mathbf{m} \mid n}^{+}$. Then the following statements are equivalent:

(a) $N$ has a dual Verma flag;

(b) $\operatorname{Ext}^{i}\left(K_{n}(\lambda), N\right)=0$ for all $\lambda \in X_{\mathbf{m} \mid n}^{+}$and all $i>0$;

(c) $\operatorname{Ext}^{1}\left(K_{n}(\lambda), N\right)=0$ for all $\lambda \in X_{\mathbf{m} \mid n}^{+}$.

(3) A tilting module in $\mathcal{O}_{\mathbf{m} \mid n}^{+}$has a finite dual Verma flag.

Proof. Part (2) can be proved using (1) exactly as for [Jan, Proposition 4.16]. Part (3) follows from (2) (also see [Br2]).

So it remains to prove (1). Using an induction on the Verma flag length on $M$ and then an induction on the dual Verma flag length on $N$, it suffices to show that $\operatorname{Ext}^{i}\left(K_{n}(\lambda), K_{n}(\mu)^{\tau}\right)=0$ for all $\lambda, \mu$ and $i \geq 1$.

As in [Br2, Lemma 3.6 (iii)], we have $\operatorname{Ext}^{i}\left(K_{n}(\lambda), K_{n}(\mu)^{\tau}\right)=0$ with $i=1$. The Ext ${ }^{i}$ vanishing for $i>1$ follows by a standard induction argument, which we sketch below for the convenience of the reader. We have an exact sequence

$$
0 \rightarrow K \rightarrow P_{n}(\lambda) \rightarrow K_{n}(\lambda) \rightarrow 0,
$$

where $K$ has a finite Verma flag. We get a long exact sequence

$$
\begin{aligned}
\cdots \leftarrow \operatorname{Ext}^{i+1}\left(P_{n}(\lambda), K_{n}(\mu)^{\tau}\right) & \leftarrow \operatorname{Ext}^{i+1}\left(K_{n}(\lambda), K_{n}(\mu)^{\tau}\right) \\
& \leftarrow \operatorname{Ext}^{i}\left(K, K_{n}(\mu)^{\tau}\right) \leftarrow \cdots .
\end{aligned}
$$

Note that $\operatorname{Ext}^{i+1}\left(P_{n}(\lambda), K_{n}(\mu)^{\tau}\right)=0$, since $P_{n}(\lambda)$ is projective. By inductive assumption, $\operatorname{Ext}^{i}\left(K_{n}(\nu), K_{n}(\mu)^{\tau}\right)=0$ for all $\nu$, and thus $\operatorname{Ext}^{i}\left(K, K_{n}(\mu)^{\tau}\right)=0$ by induction on the Verma flag length of $K$. Hence, $\operatorname{Ext}^{i+1}\left(K_{n}(\lambda), K_{n}(\mu)^{\tau}\right)=$ 0. 
Corollary 3.8. We have $U_{n}(\lambda) \cong U_{n}(\lambda)^{\tau}$.

Proof. We have $\operatorname{Ext}^{1}\left(U_{n}(\lambda), K_{n}(\mu)^{\tau}\right)=0$ by Proposition 3.7 (1), and hence by applying the functor $\tau, \operatorname{Ext}^{1}\left(K_{n}(\mu), U_{n}(\mu)^{\tau}\right)=0$. By the construction of tilting modules [So2], $\operatorname{Hom}_{\mathbf{m} \mid n}\left(K_{n}(\mu), U_{n}(\lambda)\right)=0$, for $\mu \succ \lambda$, and $\operatorname{Hom}_{\mathbf{m} \mid n}\left(K_{n}(\lambda), U_{n}(\lambda)\right)=1$. Thus there are no weights in $U_{n}(\lambda)^{\tau}$ greater than $\lambda$, which appears with multiplicity one. Now $U_{n}(\lambda)^{\tau}$ also has a Verma flag by Proposition 3.7. Thus $U_{n}(\lambda)^{\tau} \cong U_{n}(\lambda)$ by uniqueness of tilting modules.

\section{§3.4. A parabolic version of the Brundan conjecture}

The same arguments as in [Br2, So2] give us the following:

$$
\left(U_{n}(\lambda): K_{n}(\mu)\right)=\left[K_{n}\left(-w_{0} \mu-2 \rho+2 \rho_{\mathfrak{l}}\right): L_{n}\left(-w_{0} \lambda-2 \rho+2 \rho_{\imath}\right)\right],
$$

where we recall that $w_{0}$ is the longest element in the Weyl group $S_{\mathbf{m} \mid n}$ of the Levi subalgebra $\mathfrak{l}$, and $\rho_{\mathfrak{l}}$ is half the sum of positive roots of $\mathfrak{l}$.

It is well known that each $\lambda \in X_{\mathbf{m} \mid n}^{+}$(or more generally $\lambda \in \mathfrak{h}^{*}$ ) gives rise to a central character $\chi_{\lambda}$. There is a neat characterization of central characters in terms of $\epsilon$-weights [Br1, Lemma 4.18]: $\chi_{\lambda}=\chi_{\mu}$ for $\lambda, \mu \in X_{\mathbf{m} \mid n}^{+}$if and only if $\mathrm{wt}^{\epsilon}\left(f_{\lambda}\right)=\mathrm{wt}^{\epsilon}\left(f_{\mu}\right)$. It follows that the category $\mathcal{O}_{\mathbf{m} \mid n}^{+}$has a "block" decomposition $\mathcal{O}_{\mathbf{m} \mid n}^{+}=\sum_{\gamma \in P} \mathcal{O}_{\gamma}^{+}$. Denote the projection functor to $O_{\gamma}^{+}$by $\operatorname{pr}_{\gamma}$.

Let $V$ be the natural $\mathfrak{g l}(m \mid n)$-module and $V^{*}$ its dual. For $a \in \mathbb{Z}, r \geq 1$ we define the translation functors $E_{a}^{(r)}, F_{a}^{(r)}: \mathcal{O}_{\mathbf{m} \mid n}^{+} \longrightarrow \mathcal{O}_{\mathbf{m} \mid n}^{+}$by sending $M \in \mathcal{O}_{\gamma}^{+}$ to

$$
\begin{aligned}
& F_{a}^{(r)} M:=\operatorname{pr}_{\gamma-r\left(\epsilon_{a}-\epsilon_{a+1}\right)}\left(S^{r} V \otimes M\right), \\
& E_{a}^{(r)} M:=\operatorname{pr}_{\gamma+r\left(\epsilon_{a}-\epsilon_{a+1}\right)}\left(S^{r} V^{*} \otimes M\right) .
\end{aligned}
$$

By convention, set $F_{a}=F_{a}^{(1)}, E_{a}=E_{a}^{(1)}$. Let $\mathcal{O}_{\mathbf{m} \mid n}^{+, \Delta}$ be the full subcategory of $\mathcal{O}_{\mathbf{m} \mid n}^{+}$consisting of all modules with Verma flags. Let $G\left(\mathcal{O}_{\mathbf{m} \mid n}^{+, \Delta}\right)_{\mathbb{Q}}:=G\left(\mathcal{O}_{\mathbf{m} \mid n}^{+, \Delta}\right) \otimes_{\mathbb{Z}}$ $\mathbb{Q}$ and let $\left.\mathcal{E}^{\mathbf{m} \mid n}\right|_{q=1}$ be the specialization of $\mathcal{E}^{\mathbf{m} \mid n}$ as $q \rightarrow 1$. Denote the $q \rightarrow 1$ specialization of $U_{f}, K_{f}$ by $U_{f}(1), K_{f}(1)$ et cetera.

Theorem 3.9. Let $n \in \mathbb{N}$.

(1) Sending the Chevalley generators $E_{a}^{(r)}, F_{a}^{(r)}(a \in \mathbb{Z}, r \geq 1)$ to the translation functors $E_{a}^{(r)}, F_{a}^{(r)}$ defines a $\mathcal{U}_{q=1}$-module structure on $G\left(\mathcal{O}_{\mathbf{m} \mid n}^{+, \Delta}\right)_{\mathbb{Q}}$.

(2) The linear map $i:\left.G\left(\mathcal{O}_{\mathbf{m} \mid n}^{+, \Delta}\right)_{\mathbb{Q}} \rightarrow \mathcal{E}^{\mathbf{m} \mid n}\right|_{q=1}$, which sends $\left[K_{n}(\lambda)\right]$ to $K_{f_{\lambda}}(1)$, for each $\lambda \in X_{\mathbf{m} \mid n}^{+}$, is an isomorphism of $\mathfrak{U}_{q=1}$-modules. 
Proof. This is a straightforward generalization of [Br1, Theorems 4.28, $4.29]$, and it can be proved similarly.

The following is a parabolic version of [Br1, Conjecture 4.32].

Conjecture 3.10. [Parabolic Brundan-Kazhdan-Lusztig Conjecture] Let $n \in \mathbb{N} \cup \infty$. The map $i:\left.G\left(\mathcal{O}_{\mathbf{m} \mid n}^{+, \Delta}\right)_{\mathbb{Q}} \rightarrow \mathcal{E}^{\mathbf{m} \mid n}\right|_{q=1}$ sends $\left[U_{n}(\lambda)\right]$ to $U_{f_{\lambda}}(1)$ for each $\lambda \in X_{\mathbf{m} \mid n}^{+}$. (The case for $n=\infty$ will be clarified and made plausible by Theorem 3.14 below.)

Conjecture 3.10 can be equivalently reformulated as either of the following conjectural identities, in light of (2.4), (3.2), and Theorem 3.9: for $\lambda, \mu \in X_{\mathbf{m} \mid n}^{+}$,

$$
\begin{aligned}
\left(U_{n}(\lambda): K_{n}(\mu)\right) & =u_{\mu, \lambda}(1) \\
{\left[K_{n}(\lambda): L_{n}(\mu)\right] } & =u_{-w_{0} \lambda-2 \rho+2 \rho_{\mathrm{l}},-w_{0} \mu-2 \rho+2 \rho_{\mathrm{l}}}(1) \\
\operatorname{ch} L_{n}(\lambda) & =\sum_{\mu \in X_{\mathbf{m} \mid n}^{+}} \ell_{\mu, \lambda}(1) \operatorname{ch} K_{n}(\mu)
\end{aligned}
$$

We note that the validity of [Br1, Conjecture 4.32] would imply Conjecture 3.10 .

Remark 3.11. Let $\mathbf{n}=\left(n_{1}, \ldots, n_{r}\right) \in \mathbb{N}^{r}$ with $n=\sum_{b=1}^{r} n_{b}$. One can formulate the more general category $\mathcal{O}_{+}^{\mathbf{m} \mid \mathbf{n}}$ of $\mathfrak{g l}(m \mid n)$-modules which are semisimple over $\oplus_{a=1}^{s} \mathfrak{g l}\left(m_{a}\right) \oplus \oplus_{b=1}^{r} \mathfrak{g l}\left(n_{b}\right)$. All the statements on tilting modules and the Brundan-Kazhdan-Lusztig Conjecture in Subsections 3.3 and 3.4 can be readily generalized to this more general setup (cf. Remark 2.4). Brundan's conjecture [Br1] was formulated for the full category $\mathcal{O}$, i.e. when all $m_{a}$ and $n_{b}$ are equal to 1 .

On the other hand, the BKL conjecture on the irreducible characters in any parabolic category would follow from the validity of the corresponding Brundan's conjecture for the full category $\mathcal{O}$ (using the same argument as for the usual Lie algebras of type $A$ ).

\section{§3.5. $\quad$ Tilting modules with $n$ varied}

Proposition 3.12. For $\lambda \in X_{m \mid n+1}^{++}$the truncation functor $\mathfrak{t r}_{n}$ sends $U_{n+1}(\lambda)$ to $U_{n}(\lambda)$ if $\left(\lambda \mid \delta_{n+1}\right)=0$, and to 0 otherwise.

Proof. By the construction of tilting modules (cf. [So2, Br2]), $U_{n+1}(\lambda)$ has a Verma flag with subquotients isomorphic to $K_{n+1}(\mu)$ with $\mu \preccurlyeq \lambda$. If $\left(\lambda \mid \delta_{n+1}\right)>0$, then $\left(\mu \mid \delta_{n+1}\right)>0$ and thus $\mathfrak{t r}_{n}\left(U_{n+1}(\lambda)\right)=0$ by Lemma 3.3. 
Thanks to Lemma 3.4, the truncation functor $\mathfrak{t r}_{n}$ preserves Verma flags. It follows from the commutativity of $\tau$ with $\mathfrak{t r}_{n}$ and Proposition 3.7 that $\mathfrak{t r}_{n}$ also preserves the dual Verma flags. By Proposition 3.7, $\operatorname{Ext}^{1}\left(K_{n}(\mu), \mathfrak{t r}_{n}\left(U_{n+1}(\lambda)\right)\right.$ $=0$. If $\left(\lambda \mid \delta_{n+1}\right)=0$, then $\mathfrak{t r}_{n}\left(K_{n+1}(\lambda)\right)=K_{n}(\lambda)$ and clearly $K_{n}(\lambda)$ sits at the bottom of $\mathfrak{t r}_{n}\left(U_{n+1}(\lambda)\right)$.

To show that $\mathfrak{t r}_{n}\left(U_{n+1}(\lambda)\right)=U_{n}(\lambda)$, it remains to show that $\mathfrak{t r}_{n}\left(U_{n+1}(\lambda)\right)$ is indecomposable. Indeed, this follows by the same argument for [Don, Proposition 1.5] with the help of Proposition 3.7. We recall here that the counterpart in our setup of ([Don, Proposition 1.5] states that $\operatorname{Hom}_{\mathbf{m} \mid n+1}(M, N) \rightarrow$ $\operatorname{Hom}_{\mathbf{m} \mid n}\left(\mathfrak{t r}_{n} M, \mathfrak{t r}_{n} N\right)$ is surjective, for $M$ (respectively $N$ ) with a finite Verma (respectively dual Verma) flag. Its proof is elementary and uses induction on the (dual) Verma length, Lemma 3.4, and the standard fact that

$$
\operatorname{Hom}_{\mathbf{m} \mid n}\left(K_{n}(\lambda), K_{n}(\mu)^{\tau}\right) \cong \delta_{\lambda, \mu} \mathbb{C} .
$$

Thus, $\operatorname{End}_{\mathbf{m} \mid n}\left(\mathfrak{t r}_{n} U_{n+1}(\lambda)\right)$, as a quotient of the local $\mathbb{C}$-algebra $\operatorname{End}_{\mathbf{m} \mid n+1}\left(U_{n+1}(\lambda)\right)$, is local. This implies that $\mathfrak{t r}_{n}\left(U_{n+1}(\lambda)\right)$ is indecomposable.

Proposition 3.13. For $\lambda, \mu \in X_{\mathbf{m} \mid n}^{++}$and $n^{\prime} \geq n$, we also regard $\lambda, \mu \in$ $X_{\mathbf{m} \mid n^{\prime}}^{++}$by adjoining zeros. Then, $\left(U_{n}(\lambda): K_{n}(\mu)\right)=\left(U_{n^{\prime}}(\lambda): K_{n^{\prime}}(\mu)\right)$.

Proof. The proof is similar to the proof for Proposition 3.5, now with the help of Lemma 3.4 and Proposition 3.12.

Theorem 3.14. Let $\lambda \in X_{\mathbf{m} \mid \infty}^{+}$.

(1) There exists a unique (up to isomorphism) tilting module $U(\lambda)$ in $\mathcal{O}_{\mathbf{m} \mid \infty}^{++}$ with $K(\lambda)$ sitting at the bottom of a Verma flag. Moreover, $U(\lambda)=$ $\cup_{n} U_{n}(\lambda)$.

(2) The functor $\operatorname{tr}_{n}$ sends $U(\lambda)$ to $U_{n}(\lambda)$ if $\left(\lambda \mid \delta_{n+1}\right)=0$ and to 0 otherwise.

(3) We have $(U(\lambda): K(\mu))=\left(U_{n}(\lambda): K_{n}(\mu)\right)$ for $n \gg 0$.

(4) The Verma flag length for $U(\lambda)$ and $U_{n}(\lambda)$ for $n \gg 0$ is the same (and finite).

Proof. We define $U(\lambda)$ to be $\cup_{n} U_{n}(\lambda)$. The same proof for [CWZ, Theorem 3.16] applies here to prove (1) and (2), with the help of Proposition 3.13 above. (3) and (4) follow by an argument similar to the proof of Proposition 3.6 . 
Remark 3.15. Conjecture 3.10 as $n$ varies is compatible with the properties of truncation maps and the truncation functors (cf. Corollary 2.6 and Proposition 3.12).

\section{§4. Kazhdan-Lusztig Theory for $\mathfrak{g l}(m+n)$ Revisited and Super Duality}

\section{§4.1. Kazhdan-Lusztig polynomials and canonical basis for $\mathcal{E}^{\mathbf{m}+n}$}

In this subsection we give a presentation of certain parabolic KazhdanLusztig polynomials in terms of the Fock space $\mathcal{E}^{\mathbf{m}+n}$ (compare [FKK, Br1, $\mathrm{BKl}])$.

For $n \in \mathbb{N}$ let $\mathcal{E}_{+}^{\mathbf{m}+n}$ denote the subspace of $\mathcal{E}^{\mathbf{m}+n}$ spanned by elements of the form $\mathcal{K}_{f}, f \in \mathbb{Z}_{++}^{\mathbf{m}+n}$. For $n^{\prime}>n$ define the truncation map $\operatorname{Tr}_{n^{\prime}, n}$ : $\mathcal{E}_{+}^{\mathbf{m}+n^{\prime}} \longrightarrow \mathcal{E}_{+}^{\mathbf{m}+n}$ by sending $\mathcal{K}_{f}$ to $\mathcal{K}_{f(n)}$ if $f(i+1)=-i$ for all $i \geq n$, and to 0 otherwise. This gives rise to $\operatorname{Tr}_{n}: \mathcal{E}^{\mathbf{m}+\infty} \rightarrow \mathcal{E}_{+}^{\mathbf{m}+n}$, for all $n$, which in turn allows us to define a topological completion $\widehat{\mathcal{E}}^{\mathbf{m}+\infty}:=\lim _{n} \mathcal{E}_{+}^{\mathbf{m}+n}$, similarly as in [CWZ, 4.2]. For a finite $n$ let $\widehat{\mathcal{E}}^{\mathbf{m}+n} \equiv \mathcal{E}^{\mathbf{m}+n}$.

The following proposition can be established similarly as [Br1, Theorems 2.14 and 3.5] for the special cases $\mathbf{m}=(1, \ldots, 1)$ or $\mathbf{m}=m$.

Proposition 4.1. Let $n \in \mathbb{N} \cup \infty$. There exists a unique anti-linear bar map ${ }^{-}: \widehat{\mathcal{\varepsilon}}^{\mathbf{m}+n} \rightarrow \widehat{\mathcal{\varepsilon}}^{\mathbf{m}+n}$ such that

(1) $\overline{\mathcal{K}_{f}}=\mathcal{K}_{f}$, for all $f \in \mathbb{Z}_{+}^{\mathbf{m}+n}$ minimal in the Bruhat ordering.

(2) $\overline{X u}=\bar{X} \bar{u}$, for all $X \in \mathcal{U}$ and $u \in \widehat{\mathcal{E}}^{\mathbf{m}+n}$.

(3) The bar map is an involution.

(4) $\overline{\mathcal{K}_{f}}=\mathcal{K}_{f}+(*)$, where $(*)$ is a (possibly infinite when $\left.n=\infty\right) \mathbb{Z}\left[q, q^{-1}\right]$ linear combination of $\mathcal{K}_{g}$ 's with $g \in \mathbb{Z}_{+}^{\mathbf{m}+n}$ such that $g<f$ in the Bruhat ordering.

The next theorem follows from Proposition 4.1.

Theorem 4.2. Let $n \in \mathbb{N} \cup \infty$. There exist unique topological bases $\left\{\mathfrak{U}_{f}\right\},\left\{\mathcal{L}_{f}\right\}$, where $f \in \mathbb{Z}_{+}^{\mathbf{m}+n}$, for $\widehat{\mathcal{E}}^{\mathbf{m}+n}$ such that

(1) $\overline{\mathcal{U}}_{f}=\mathcal{U}_{f}$ and $\overline{\mathcal{L}}_{f}=\mathcal{L}_{f}$;

(2) $\mathcal{U}_{f} \in \mathcal{K}_{f}+\widehat{\sum}_{g \in \mathbb{Z}_{+}^{\mathbf{m}+n}} q \mathbb{Z}[q] \mathcal{K}_{g}$ and $\mathcal{L}_{f} \in \mathcal{K}_{f}+\widehat{\sum}_{g \in \mathbb{Z}_{+}^{\mathbf{m}+n}} q^{-1} \mathbb{Z}\left[q^{-1}\right] \mathcal{K}_{g}$. 
(3) $\mathcal{U}_{f}=\mathcal{K}_{f}+(*)$ and $\mathcal{L}_{f}=\mathcal{K}_{f}+(* *)$, where $(*)$ and $(* *)$ are (possibly infinite when $n=\infty) \mathbb{Z}\left[q, q^{-1}\right]$-linear combinations of $\mathcal{K}_{g}$ 's with $g \in \mathbb{Z}_{+}^{\mathbf{m}+n}$ such that $g<f$. For $n$ finite, $(*)$ and $(* *)$ are always finite sums.

We define $\mathfrak{u}_{g, f}(q) \in \mathbb{Z}[q], \mathfrak{l}_{g, f}(q) \in \mathbb{Z}\left[q^{-1}\right]$ for $f, g \in \mathbb{Z}_{+}^{\mathbf{m}+n}$ by

$$
\mathfrak{U}_{f}=\sum_{g \in \mathbb{Z}_{+}^{\mathbf{m}+n}} \mathfrak{u}_{g, f}(q) \mathcal{K}_{g}, \quad \mathcal{L}_{f}=\sum_{g \in \mathbb{Z}_{+}^{\mathbf{m}+n}} \mathfrak{l}_{g, f}(q) \mathcal{K}_{g} .
$$

Note that $\mathfrak{u}_{g, f}(q)=\mathfrak{l}_{g, f}(q)=0$ unless $g \leq f$ and $\mathfrak{u}_{f, f}(q)=\mathfrak{l}_{f, f}(q)=1$. These polynomials can be identified as (parabolic) Kazhdan-Lusztig polynomials (cf. Theorem 4.14 below).

Remark 4.3. By the same type of arguments as in [Br1, §3-c] we can introduce a symmetric bilinear form $\langle\cdot, \cdot\rangle$ on $\mathcal{E}^{\mathbf{m}+n}$ such that $\left\langle\mathcal{L}_{f}, \mathcal{U}_{-g \cdot w_{0}}\right\rangle=\delta_{f, g}$ for $f, g \in \mathbb{Z}_{+}^{\mathbf{m}+n}$, which readily implies that the matrices $\left[\mathfrak{u}_{-f \cdot w_{0},-g \cdot w_{0}}(q)\right]$ and $\left[\mathfrak{l}_{f, g}\left(q^{-1}\right)\right]$ are inverses of each other. Equivalently, we have

$\mathcal{K}_{f}=\sum_{g \in \mathbb{Z}_{+}^{\mathrm{m}+n}} \mathfrak{u}_{-f \cdot w_{0},-g \cdot w_{0}}\left(q^{-1}\right) \mathcal{L}_{g}=\sum_{g \in \mathbb{Z}_{+}^{\mathrm{m}+n}} \mathfrak{l}_{-f \cdot w_{0},-g \cdot w_{0}}\left(q^{-1}\right) \mathfrak{U}_{g}, \quad f \in \mathbb{Z}_{+}^{\mathbf{m}+n}$.

\section{Proposition 4.4.}

(1) The truncation map $\operatorname{Tr}_{n^{\prime}, n}: \mathcal{E}_{+}^{\mathbf{m}+n^{\prime}} \rightarrow \mathcal{E}_{+}^{\mathbf{m}+n}$ commutes with the barinvolution, where $\infty \geq n^{\prime}>n$.

(2) $\operatorname{Tr}_{n^{\prime}, n}$ sends $\mathcal{U}_{f}\left(\right.$ respectively $\left.\mathcal{L}_{f}\right)$ to $\mathcal{U}_{f^{(n)}}\left(\right.$ respectively $\left.\mathcal{L}_{f^{(n)}}\right)$ if $f(i+1)=$ $-i$, for all $i \geq n$, and to 0 otherwise.

(3) For $f, g \in \mathbb{Z}_{++}^{\mathbf{m}+n^{\prime}}$ such that $f(i+1)=g(i+1)=-i$ for all $i \geq n$, we have

$$
\mathfrak{u}_{g, f}(q)=\mathfrak{u}_{g^{(n)}, f^{(n)}}(q), \quad \mathfrak{l}_{g, f}(q)=\mathfrak{l}_{g^{(n)}, f^{(n)}}(q) .
$$

Proof. Part (1) is proved similarly as [CWZ, Proposition 4.29]. (2) and (3) are immediate corollaries.

\section{$\S 4.2$. A Fock space isomorphism and consequences}

\section{Proposition 4.5.}

(1) There is an isomorphism of U-modules $C: \Lambda^{\infty} \mathbb{V} \rightarrow \Lambda^{\infty} \mathbb{V}^{*}$ which sends $|\lambda\rangle$ to $\left|\lambda_{*}^{\prime}\right\rangle$ for each partition $\lambda$. 
(2) The map $C$ extends naturally to an isomorphism of $\mathcal{U}$-modules

$$
\natural: \widehat{\mathcal{E}}^{\mathbf{m}+\infty} \stackrel{\cong}{\cong} \widehat{\mathcal{\varepsilon}}^{\mathbf{m} \mid \infty},
$$

which is compatible with the actions of all divided powers $E_{a}^{(s)}, F_{a}^{(s)}$.

Proof. Part (1) above is [CWZ, Theorem 6.3]. Recall that $\mathcal{E}^{\mathbf{m}+\infty}=$ $\otimes_{i=1}^{s} \Lambda^{m_{i}} \mathbb{V} \otimes \Lambda^{\infty} \mathbb{V}$ and $\mathcal{E}^{\mathbf{m} \mid \infty}=\otimes_{i=1}^{s} \Lambda^{m_{i}} \mathbb{V} \otimes \Lambda^{\infty} \mathbb{V}^{*}$. Then $C: \Lambda^{\infty} \mathbb{V} \rightarrow \Lambda^{\infty} \mathbb{V}^{*}$ induces a $U$-module isomorphism $\sharp=1 \otimes C: \mathcal{E}^{\mathbf{m}+\infty} \stackrel{\cong}{\cong} \mathcal{E}^{\mathbf{m} \mid \infty}$. One can further check that these two topological completions $\widehat{\varepsilon}^{\mathbf{m}+\infty}$ and $\widehat{\mathcal{E}}^{\mathbf{m} \mid \infty}$ are indeed compatible under $\sharp$.

Given $\lambda=\sum_{i \in I(m \mid \infty)} \lambda_{i} \delta_{i}^{\prime} \in X_{\mathbf{m}+\infty}^{+}$so that by definition $\lambda^{>0}:=$ $\left(\lambda_{1}, \lambda_{2}, \ldots\right)$ is a partition. Denoting by $\left(\lambda_{1}^{\prime}, \lambda_{2}^{\prime}, \ldots\right)$ the conjugate partition of $\lambda^{>0}$, we define a weight

$$
\lambda^{\natural}:=\sum_{i=-m}^{-1} \lambda_{i} \delta_{i}+\sum_{j=1}^{\infty} \lambda_{j}^{\prime} \delta_{j} \in X_{\mathbf{m} \mid \infty}^{+} .
$$

This actually defines bijections (denoted by $₫$ by abuse of notation)

$$
X_{\mathbf{m}+\infty}^{+} \stackrel{\natural}{\longleftrightarrow} X_{\mathbf{m} \mid \infty}^{+}, \quad \mathbb{Z}_{+}^{\mathbf{m}+\infty} \stackrel{\natural}{\longleftrightarrow} \mathbb{Z}_{+}^{\mathbf{m} \mid \infty},
$$

when coupling with the two bijections $X_{\mathbf{m} \mid \infty}^{+} \leftrightarrow \mathbb{Z}_{+}^{\mathbf{m} \mid \infty}$ and $X_{\mathbf{m}+\infty}^{+} \leftrightarrow \mathbb{Z}_{+}^{\mathbf{m}+\infty}$. There is a simple combinatorial description for the bijection

$$
\mathbb{Z}_{+}^{\mathbf{m}+\infty} \stackrel{\natural}{\longrightarrow} \mathbb{Z}_{+}^{\mathbf{m} \mid \infty}, \quad\left(f^{<0} \mid f^{>0}\right) \mapsto f=\left(f^{<0} \mid \mathbb{Z} \backslash f^{>0}\right)
$$

in light of [CWZ, Lemma 6.2], where $f^{>0}$ denotes the restriction of $f$ to $I(0 \mid \infty)$ and $\mathbb{Z} \backslash f^{>0}$ denotes the complement of $f^{>0}$ in $\mathbb{Z}$.

\section{Lemma 4.6.}

(1) For $f, g \in \mathbb{Z}_{+}^{\mathbf{m}+\infty}, f \geq g$ in the Bruhat ordering if and only if $f^{\natural} \succcurlyeq g^{\natural}$ in the super Bruhat ordering.

(2) A weight $\lambda \in X_{m+\infty}^{+}$is minimal in the Bruhat ordering if and only if $\lambda^{\natural} \in X_{\mathbf{m} \mid \infty}^{+}$is minimal in the super Bruhat ordering.

Proof. (2) is a special case of (1), so let us prove (1).

Denote by $f^{+}$the (unique if exists) conjugate in $\mathbb{Z}_{+}^{m+\infty}$ of $f \in \mathbb{Z}^{m+\infty}$ under the action of $S_{\mathbf{m}+\infty}$. The super Bruhat ordering $\succcurlyeq$ on $\mathbb{Z}_{+}^{\mathbf{m} \mid \infty}$ is the transitive closure of the partial order $f \succcurlyeq g$ given by 
(i) $g=\left(f-d_{i}+d_{j}\right)^{+}$for some $i<0<j$ such that $f(i)=f(j)$;

(ii) $g=\left(f \cdot \tau_{i j}\right)^{+}$for some $i<j<0$ such that $f(i)>f(j)$.

On the other hand, the Bruhat ordering $\geq$ on $\mathbb{Z}_{+}^{\mathbf{m}+\infty}$ is the transitive closure of the partial order $f \geq g$ given by

(i') $g=\left(f \cdot \tau_{i j}\right)^{+}$for some $i<0<j$ such that $f(i)>f(j)$;

(ii') $g=\left(f \cdot \tau_{i j}\right)^{+}$for some $i<j<0$ such that $f(i)>f(j)$.

Exactly as explained in the proof of [CWZ, Lemma 6.6] when $\mathbf{m}=m$, under the explicit bijection $\downarrow: \mathbb{Z}_{+}^{\mathbf{m}+\infty} \longrightarrow \mathbb{Z}_{+}^{\mathbf{m} \mid \infty}$ given by (4.2), the Step (i) corresponds to Step (i'). Now clearly the Step (ii) corresponds to (ii') by (4.2). This proves (1).

Theorem 4.7. The isomorphism $\natural: \widehat{\mathcal{E}}^{\mathbf{m}+\infty} \longrightarrow \widehat{\mathcal{E}}^{\mathbf{m}} \mid \infty$ has the following properties:

(1) $\natural\left(\mathcal{K}_{f}\right)=K_{f}$ for each $f \in \mathbb{Z}_{+}^{\mathbf{m}+\infty}$;

(2) $\square$ is compatible with the bar involutions, i.e., $\square(\bar{u})=\overline{\natural(u)}$ for each $u \in$ $\widehat{\mathcal{E}}^{\mathbf{m}+\infty}$

(3) $\natural\left(\mathcal{L}_{f}\right)=L_{f^{\natural}}$ for each $f \in \mathbb{Z}_{+}^{\mathbf{m}+\infty}$;

(4) $\natural\left(\mathcal{U}_{f}\right)=U_{f \natural}$ for each $f \in \mathbb{Z}_{+}^{\mathbf{m}+\infty}$.

(5) For $f, g \in \mathbb{Z}_{+}^{\mathbf{m}+\infty}$, we have $\mathfrak{u}_{g, f}(q)=u_{g^{\natural}, f^{\natural}}(q)$, and $\mathfrak{l}_{g, f}(q)=\ell_{g^{\natural}, f^{\natural}}(q)$.

Proof. (1) follows from the definitions and Proposition 4.5. (2) follows from Proposition 4.5, Lemma 4.6 (2) and the characterizations of the bar involutions. (3) and (4) follow from (1), (2), Lemma 4.6, and the characterizations of these bases.

The following verifies a parabolic version of [Br1, Conjecture 2.28].

\section{Theorem 4.8 .}

(1) The Brundan-Kazhdan-Lusztig polynomials satisfy the following positivity: $\mathfrak{u}_{\mu, \lambda}(q) \in \mathbb{N}[q], \mathfrak{l}_{\mu, \lambda}\left(-q^{-1}\right) \in \mathbb{N}[q]$ for all $\lambda, \mu \in X_{\mathbf{m} \mid n}^{+}$.

(2) For each $a \in \mathbb{Z}, r \geq 1$, and $f \in \mathbb{Z}_{+}^{\mathbf{m} \mid n}$, the coefficients of $E_{a}^{(r)} U_{f}, F_{a}^{(r)} U_{f}$ (respectively $E_{a}^{(r)} L_{f}, F_{a}^{(r)} L_{f}$ ) in the expansion in terms of the canonical basis $\left\{U_{g}\right\}$ (respectively, the dual canonical basis $\left\{L_{g}\right\}$ ) lie in $\mathbb{N}\left[q, q^{-1}\right]$. 
Remark 4.9. Set $n=0$ in Theorem 4.8, and we are in the setup of the Fock space corresponding to usual parabolic category $\mathcal{O}_{\mathbf{m}}^{+}$of $\mathfrak{g l}(m)$-modules. It is folklore that Theorem 4.8 (2) with $n=0$ should be true and indeed a proof is known to Lusztig [Lu3]. Theorem 4.8 (2) with $n=0$ would also follow from the graded lifts in the sense of Beilinson, Ginzburg and Soergel [BGS] of the category $\mathcal{O}_{\mathbf{m}}^{+}$and the divided power translation functors $E_{a}^{(r)}, F_{a}^{(r)}$, for $a \in \mathbb{Z}, r \geq 1$. For example, a complete proof in a special case of such a lift of the divided powers has been written down by Frenkel, Khovanov and Stroppel [FKS, Theorems 3.6, 5.3] (see Remark 5.6 therein for the general category $\mathcal{O}$, and the parabolic case should follow too). We thank Jon Brundan for the reference and clarification.

Proof. (1) It suffices to prove when $n$ is finite. Let us identify the Kazhdan-Lusztig polynomials for $\mathfrak{g l}(m \mid n)$ with the usual Kazhdan-Lusztig polynomials for $\mathfrak{g l}(m+N)$ for finite $n$ and $N$. Given $\lambda, \mu \in X_{\mathbf{m} \mid n}^{++}$, we obtain $\lambda_{\infty} \in X_{\mathbf{m} \mid \infty}^{+}$the extension of $\lambda$ by zeros, and $\lambda_{\infty}^{\natural} \in X_{\mathbf{m}+\infty}^{+}$. Write $\lambda_{\infty}^{\natural}=$ $\left(\left(\lambda_{\infty}^{\natural}\right)^{<0} \mid\left(\lambda_{\infty}^{\natural}\right)^{>0}\right)$. Assuming the lengths of the partitions $\left(\mu_{\infty}^{\natural}\right)^{>0}$ and $\left(\lambda_{\infty}^{\natural}\right)^{>0}$ are no larger than $N$, we have $\lambda_{\infty}^{\natural,(N)}, \mu_{\infty}^{\natural,(N)} \in X_{\mathbf{m}+N}^{++}$. Then,

$$
u_{\mu, \lambda}(q)=u_{\mu_{\infty}, \lambda_{\infty}}(q)=\mathfrak{u}_{\mu_{\infty}^{\natural}, \lambda_{\infty}^{\natural}}(q)=\mathfrak{u}_{\mu_{\infty}^{\natural,(N)}, \lambda_{\infty}^{\natural,(N)}}(q) .
$$

Similarly, we have $\ell_{\mu, \lambda}(q)=\mathfrak{l}_{\mu_{\infty}^{\natural,(N)}, \lambda_{\infty}^{\natural,(N)}}(q)$.

The general case of $u_{\mu, \lambda}(q), \ell_{\mu, \lambda}(q)$ for $\lambda, \mu \in X_{\mathbf{m} \mid n}^{+}$can be easily reduced to the case considered above as follows. Let $\mathbf{1}_{m \mid n}:=\overbrace{-1, \ldots,-1}^{m} \mid \overbrace{1, \ldots, 1}^{n} \in$ $X_{\mathbf{m} \mid n}^{++}$. Note that $u_{\mu, \lambda}(q)=u_{\mu+k \mathbf{1}_{m \mid n}, \lambda+k \mathbf{1}_{m \mid n}}(q), \ell_{\mu, \lambda}(q)=\ell_{\mu+k \mathbf{1}_{m \mid n}, \lambda+k \mathbf{1}_{m \mid n}}(q)$, and also that $\lambda+k \mathbf{1}_{m \mid n} \in X_{\mathbf{m} \mid n}^{++}$, for $\lambda \in X_{\mathbf{m} \mid n}^{+}$and $k \gg 0$.

Thus our result follows from the corresponding well-known positivity results of Kazhdan-Lusztig polynomials which was proved using deep geometric techniques [KL2, BB, BK].

(2) Let $1_{m \mid n} \in \mathbb{Z}^{\mathbf{m} \mid n}$ denote the function given by $1_{m \mid n}(i)=1$, for all $i \in I(m \mid n)$. The formula for $U_{f-k 1_{m \mid n}}$, with $k \in \mathbb{Z}$, is obtained from $U_{f}$ by shifting the weights in the monomials that appear in $U_{f}$ by $-k 1_{m \mid n}$. Also if we write $X_{a}^{(r)} U_{f}=\sum_{g} x_{g f}(q) U_{g}$, with $x_{g f}(q) \in \mathbb{Z}\left[q, q^{-1}\right]$, then $X_{a-k}^{(r)} U_{f-k 1_{m \mid n}}=$ $\sum_{g} x_{g f}(q) U_{g-k 1_{m \mid n}}($ here $X=E, F)$. Thus it suffices to verify (2) within $\mathcal{E}_{+}^{\mathbf{m} \mid n}$ by assuming $a<n$ and $f \in \mathbb{Z}_{++}^{\mathbf{m} \mid n}$. Using the truncation maps we can pass to the case when $n=\infty$ (see Corollary 2.6). By Proposition 4.5 and Theorem 4.7, this amounts to prove the corresponding statement for $\mathcal{U}_{f}$ and $\mathcal{L}_{f}$ in $\widehat{\mathcal{E}}^{\mathbf{m}+\infty}$. But this follows from the validity of the corresponding statement in $\widehat{\mathcal{E}}^{\mathbf{m}+n}$ for 
$n$ finite (see Remark 4.9) and the property of the truncation map $\operatorname{Tr}_{\infty, n}$ in Proposition 4.4.

As explained in [Br1, 2-k], the positivity in Theorem 4.8 (2) together with (a parabolic variant of) the algorithm in $[\mathrm{Br} 1,2-\mathrm{j}]$ for computing the canonical basis elements in $\widehat{\mathcal{E}}^{\mathbf{m} \mid n}$ imply the following.

Corollary 4.10. Let $n$ be finite. Every canonical basis element $U_{f}$ in the completion $\widehat{\mathcal{E}}_{+}^{\mathbf{m} \mid n}$ actually lies in $\mathcal{E}_{+}^{\mathbf{m} \mid n}$, that is, $U_{f}$ is a finite sum of monomials $K_{g}$.

Remark 4.11. Such a finiteness of canonical basis elements in $\mathcal{E}^{\mathbf{m}} \mid n$ supports Conjecture 3.10, since it is compatible with the fact that a Verma flag of any tilting module in $\mathcal{O}_{\mathbf{m} \mid n}^{+}$is finite.

Corollary 4.12. Let $\infty \geq n>n_{0}, f \in \mathbb{Z}_{++}^{\mathbf{m} \mid n_{0}}$, and extend $f$ to $f^{(n)} \in$ $\mathbb{Z}_{++}^{\mathbf{m} \mid n}$ by letting $f^{(n)}(i)=i$ for $n_{0}<i \leq n$. Let $n_{f} \gg 0$ be the smallest integer such that $\# f^{(n)}=\# f$, for all $n \geq n_{f}$. Then $U_{f^{(n)}}$ contains the same (finite) number of monomials for all $\infty \geq n \geq n_{f}$.

Proof. Let $\infty \geq n \geq n_{f}$. Also write $U_{f^{(n)}}=\sum_{g \preceq f^{(n)}} u_{g, f^{(n)}}(q) K_{g}$. It follows from $g \preceq f^{(n)}$ and $n \geq n_{f}$ that $g \in X_{\mathbf{m} \mid n}^{++}$and $g=g_{1}^{(n)}$ for $g_{1} \in X_{\mathbf{m} \mid n_{f}}^{++}$. Recall that $\mathfrak{T r}_{n, n_{f}}\left(K_{g}\right)=K_{g_{1}}, \mathfrak{T}_{\mathfrak{r}_{n, n_{f}}}\left(U_{f^{(n)}}\right)=U_{f^{\left(n_{f}\right)}}$. Thus when applying the truncation map $\mathfrak{T r}_{n, n_{f}}$ to the previous identity for $U_{f^{(n)}}$, every nonzero monomial survives, and we obtain that $U_{f^{\left(n_{f}\right)}}=\sum_{g_{1} \preceq f} u_{g, f(n)}(q) K_{g_{1}}$.

Corollary 4.13. Let $n>n_{0}, f \in \mathbb{Z}_{++}^{\mathbf{m}+n_{0}}$, and extend $f$ to $f^{(n)} \in \mathbb{Z}_{++}^{\mathbf{m}+n}$ by letting $f^{(n)}(i)=1-i$ for $n_{0}<i \leq n$. Then, there exists $n_{f} \gg 0$ such that the number of monomials in $\mathcal{U}_{f^{(n)}}$ is independent of $n \geq n_{f}$.

Proof. By a truncation map argument similar to the proof of Corollary 4.12 , the number of monomial terms in $\mathcal{U}_{f^{(n)}}$ is weakly increasing as $n$ increases. But this number has to stabilize, since it is bounded according to Corollary 4.12 and Theorem 4.7 (4).

\section{§4.3. The category $\mathcal{O}_{\mathbf{m}+n}^{+}$}

Let $n \in \mathbb{N}$. We shall think of $\mathfrak{g l}(m+n)$ as the Lie algebra of complex matrices whose rows and columns are parameterized by $I(m \mid n)$. Let $e_{i j}, i, j \in$ 
$I(m \mid n)$ be the elementary matrices. We denote by $\mathfrak{h}_{c}$ (respectively $\mathfrak{b}_{c}$ ) the standard Cartan (respectively Borel) subalgebra of $\mathfrak{g l}(m+n)$, which consists of the diagonal (respectively the upper triangular) matrices. Let $\left\{\delta_{i}^{\prime}, i \in I(m \mid n)\right\}$ be the basis of $\mathfrak{h}_{c}^{*}$ dual to $\left\{e_{i i}, i \in I(m \mid n)\right\}$. Introduce the Levi subalgebra $\mathfrak{l}=\oplus_{i=1}^{s} \mathfrak{g l}\left(m_{i}\right) \oplus \mathfrak{g l}(n)$ and the corresponding parabolic subalgebra $\mathfrak{q}=\mathfrak{l}+\mathfrak{b}_{c}$ of $\mathfrak{g l}(m+n)$. Let $\mathfrak{g l}(m+\infty)=\lim _{\vec{n}} \mathfrak{g l}(m+n)$.

Define the symmetric bilinear form $(\cdot \mid \cdot)_{c}$ on $\mathfrak{h}_{c}^{*}$ by

$$
\left(\delta_{i}^{\prime} \mid \delta_{j}^{\prime}\right)_{c}=\delta_{i j}, \quad i, j \in I(m \mid n) .
$$

Let $X_{m+n}$ be the set of integral weights $\lambda=\sum_{i \in I(m \mid n)} \lambda_{i} \delta_{i}^{\prime}, \lambda_{i} \in \mathbb{Z}$. Define

$$
\begin{gathered}
X_{\mathbf{m}+n}^{+}:=\left\{\lambda \in X_{m+n} \mid \lambda_{-m} \geq \cdots \geq \lambda_{-m+m_{1}-1},\right. \\
\lambda_{-m+m_{1}} \geq \cdots \geq \lambda_{-m+m_{1}+m_{2}-1}, \\
\left.\quad \cdots, \lambda_{-m_{s}} \geq \cdots \geq \lambda_{-1}, \lambda_{1} \geq \cdots \geq \lambda_{n}\right\}, \\
X_{\mathbf{m}+n}^{++}:=\left\{\lambda \in X_{\mathbf{m}+n}^{+} \mid \lambda_{n} \geq 0\right\} .
\end{gathered}
$$

We may regard an element $\lambda$ in $X_{\mathbf{m}+n}^{++}$as an element in $X_{\mathbf{m}+n^{\prime}}^{++}$for $n^{\prime}>n$ by adjoining zeros. Set

$$
X_{\mathbf{m}+\infty}^{++} \equiv X_{\mathbf{m}+\infty}^{+}:=\lim _{\vec{n}} X_{\mathbf{m}+n}^{++} .
$$

For $n \in \mathbb{N} \cup \infty$ define

$$
\rho^{\prime}=-\sum_{i=-m}^{-1} i \delta_{i}^{\prime}+\sum_{j=1}^{n}(1-j) \delta_{j}^{\prime} .
$$

Define a bijection

$$
X_{\mathbf{m}+n} \longrightarrow \mathbb{Z}^{\mathbf{m}+n}, \quad \lambda \mapsto f_{\lambda},
$$

where $f_{\lambda} \in \mathbb{Z}^{\mathbf{m}+n}$ is given by $f_{\lambda}(i)=\left(\lambda+\rho^{\prime} \mid \delta_{i}^{\prime}\right)_{c}$ for all $i \in I(m \mid n)$. This map induces bijections $X_{\mathbf{m}+n}^{+} \rightarrow \mathbb{Z}_{+}^{\mathbf{m}+n}$, and $X_{\mathbf{m}+n}^{++} \rightarrow \mathbb{Z}_{++}^{\mathbf{m}+n}$. Using this bijection we define the notions such as $\epsilon$-weight, partial order $\leq$, et cetera, for elements in $X_{\mathbf{m}+n}^{+}$by requiring them to be compatible with those defined for elements in $\mathbb{Z}_{+}^{\mathbf{m}+n}$.

Given $\lambda \in X_{\mathbf{m}+n}^{+}, n \in \mathbb{N} \cup \infty$, we define as usual the parabolic Verma module

$$
\mathcal{K}_{n}(\lambda):=U(\mathfrak{g l}(m+n)) \otimes_{U(\mathfrak{q})} L_{n}^{0}(\lambda)
$$

and its irreducible quotient $\mathfrak{g l}(m+n)$-module $\mathcal{L}_{n}(\lambda)$. 
Let $n \in \mathbb{N}$. Denote by $\mathcal{O}_{\mathbf{m}+n}^{+}$the category of finitely generated $\mathfrak{g l}(m+n)$ modules $M$ that are locally finite over $\mathfrak{q}$, semisimple over $\mathfrak{l}$ and

$$
M=\bigoplus_{\gamma \in X_{m+n}} M_{\gamma}
$$

where as usual $M_{\gamma}$ denotes the $\gamma$-weight space of $M$ with respect to $\mathfrak{h}_{c}$. The parabolic Verma module $\mathcal{K}_{n}(\lambda)$ and the irreducible module $\mathcal{L}_{n}(\lambda)$ for $\lambda \in X_{\mathbf{m}+n}^{+}$ belong to $\mathcal{O}_{\mathbf{m}+n}^{+}$. Denote by $\mathcal{O}_{\mathbf{m}+n}^{++}$the full subcategory of $\mathcal{O}_{\mathbf{m}+n}^{+}$which consists of $\mathfrak{g l}(m+n)$-modules $M$ whose composition factors are isomorphic to $\mathcal{L}_{n}(\lambda)$ with $\lambda \in X_{\mathbf{m}+n}^{++}$. Given $M \in \mathcal{O}_{\mathbf{m}+n}^{+}$, we endow the restricted dual $M^{*}$ with the usual $\mathfrak{g l}(m+n)$-module structure. Further twisting the $\mathfrak{g l}(m+n)$-action on $M^{*}$ by the automorphism given by the negative transpose of $\mathfrak{g l}(m+n)$, we obtain another g-module denoted by $M^{\tau}$.

Tilting modules $\mathcal{U}_{n}(\lambda)$ for $\lambda \in X_{\mathbf{m}+n}^{+}$in $\mathcal{O}_{\mathbf{m}+n}^{+}$were constructed as in [CoI, So2] and are known to have Verma flags. The character formula of the tilting module $\mathcal{U}_{n}(\mu)$ in $\mathcal{O}_{\mathbf{m}+n}^{+}$is given by [So2]: for $\lambda, \mu \in X_{\mathbf{m}+n}^{+}$,

(4.4) $\left(\mathcal{U}_{n}(\lambda): \mathcal{K}_{n}(\mu)\right)=\left[\mathcal{K}_{n}\left(-w_{0} \mu-2 \rho^{\prime}+2 \rho_{\mathfrak{l}}\right): \mathcal{L}_{n}\left(-w_{0} \lambda-2 \rho^{\prime}+2 \rho_{\mathfrak{l}}\right)\right]$.

We remark that for $n \in \mathbb{N} \cup \infty$ the $\mathfrak{g l}(m+n)$-module $\mathcal{K}_{n}(\lambda)$ is irreducible if and only if $\lambda$ is a minimal weight in $X_{\mathbf{m}+n}^{+}$in the Bruhat ordering.

Denote by $\mathcal{O}_{\mathbf{m}+\infty}^{++}$the category of finitely generated $\mathfrak{g l}(m+\infty)$-modules that are l-semisimple, locally finite over $\mathfrak{q} \cap \mathfrak{g l}(m+N)$, for every $N$, and such that the composition factors are of the form $\mathcal{L}(\lambda), \lambda \in X_{\mathbf{m}+\infty}^{+}$.

\section{§4.4. Kazhdan-Lusztig theory and (dual) canonical bases}

We will write $\mathfrak{l}_{g, f}(q), \mathfrak{t}_{g, f}(q)$ for $\mathfrak{l}_{\mu, \lambda}(q), \mathfrak{t}_{\mu, \lambda}(q)$, where $f, g$ correspond to $\lambda, \mu$, respectively, under the bijection $X_{m+n}^{+} \rightarrow \mathbb{Z}_{+}^{m+n}$.

The following is an increasingly better known reformulation, in terms of dual canonical and canonical bases, of the Kazhdan-Lusztig conjecture, proved in $[\mathrm{BB}, \mathrm{BK}]$, combined with the translation principle and the character formula of tilting modules [So2]. The proof in [CWZ, Theorem 5.4] for the special case (i.e. $\mathbf{m}=m$ ) works in the current setup as well (also cf. Brundan-Kleshchev $[\mathrm{BKl}])$.

Theorem 4.14. In the Grothendieck group $G\left(\mathcal{O}_{\mathbf{m}+n}^{+}\right)$, for $\nu \in X_{m+n}^{+}$, we have

$$
\left[\mathcal{U}_{n}(\nu)\right]=\sum_{\mu \in X_{\mathbf{m}+n}^{+}} \mathfrak{u}_{\mu, \nu}(1)\left[\mathcal{K}_{n}(\mu)\right]
$$


Theorem 4.14 is equivalent to the following character formula by Remark 4.3 and (4.4):

$$
\operatorname{ch} \mathcal{L}_{n}(\nu)=\sum_{\mu \in X_{\mathbf{m}+n}^{+}} \mathfrak{l}_{\mu, \nu}(1) \operatorname{ch} \mathcal{K}_{n}(\mu) .
$$

Recall the $\epsilon$-weight on $X_{m+n}$ defined in (2.1). Denote by $\chi_{\lambda}$ the central character associated to $\lambda \in X_{m+n}$. By Harish-Chandra's theorem $\chi_{\lambda}=\chi_{\mu}$ for $\lambda, \mu \in X_{m+n}$ if and only if $\lambda=\sigma \cdot \mu$ for some $\sigma \in S_{m+n}$, or equivalently $\mathrm{wt}^{\epsilon}(\lambda)=\mathrm{wt}^{\epsilon}(\mu) \in P$. We denote by $\mathcal{O}_{\gamma}^{+}$the block in $\mathcal{O}_{\mathbf{m}+n}^{+}$associated to $\gamma \in P$, and by $\operatorname{pr}_{\gamma}$ the projection functor onto $O_{\gamma}^{+}$. Let $V$ be the natural $\mathfrak{g l}(m+n)$-module and $V^{*}$ its dual. For $a \in \mathbb{Z}, r \geq 1$ we define the translation functors $E_{a}^{(r)}, F_{a}^{(r)}: \mathcal{O}_{\mathbf{m}+n}^{+} \longrightarrow \mathcal{O}_{\mathbf{m}+n}^{+}$by sending $M \in \mathcal{O}_{\gamma}^{+}$to

$F_{a}^{(r)} M:=\operatorname{pr}_{\gamma-r\left(\epsilon_{a}-\epsilon_{a+1}\right)}\left(S^{r} V \otimes M\right), \quad E_{a}^{(r)} M:=\operatorname{pr}_{\gamma+r\left(\epsilon_{a}-\epsilon_{a+1}\right)}\left(S^{r} V^{*} \otimes M\right)$.

Let $\mathcal{O}_{\mathbf{m}+n}^{+, \Delta}$ be the full subcategory of $\mathcal{O}_{\mathbf{m}+n}^{+}$consisting of all modules with Verma flags. Let $G\left(\mathcal{O}_{\mathbf{m}+n}^{+, \Delta}\right)_{\mathbb{Q}}:=G\left(\mathcal{O}_{\mathbf{m}+n}^{+, \Delta}\right) \otimes_{\mathbb{Z}} \mathbb{Q}$ and let $\left.\mathcal{E}^{\mathbf{m}+n}\right|_{q=1}$ be the specialization of $\mathcal{E}^{\mathbf{m}+n}$ as $q \rightarrow 1$.

Theorem 4.15. Let $n \in \mathbb{N}$.

(1) Sending the Chevalley generators $E_{a}^{(r)}, F_{a}^{(r)}(a \in \mathbb{Z}, r \geq 1)$ to the translation functors $E_{a}^{(r)}, F_{a}^{(r)}$ defines a $\mathfrak{U}_{q=1}$-module structure on $G\left(\mathcal{O}_{\mathbf{m}+n}^{+, \Delta}\right)_{\mathbb{Q}}$.

(2) The linear map $i:\left.G\left(\mathcal{O}_{\mathbf{m}+n}^{+, \Delta}\right)_{\mathbb{Q}} \rightarrow \mathcal{E}^{\mathbf{m}+n}\right|_{q=1}$, which sends $\left[\mathcal{K}_{n}(\lambda)\right]$ to $\mathcal{K}_{f_{\lambda}}(1)$, for each $\lambda \in X_{\mathbf{m}+n}^{+}$, is an isomorphism of $\mathfrak{U}_{q=1}$-modules.

(3) The map $i$ sends $\left[\mathcal{U}_{n}(\lambda)\right]$ to $\mathcal{U}_{f_{\lambda}}(1)$, for each $\lambda \in X_{\mathbf{m}+n}^{+}$.

Equivalently, for $\lambda, \mu \in X_{\mathbf{m}+n}^{+}$, we have

$$
\begin{aligned}
\left(\mathcal{U}_{n}(\lambda): \mathcal{K}_{n}(\mu)\right) & =\mathfrak{u}_{\mu, \lambda}(1), \\
{\left[\mathcal{K}_{n}(\lambda): \mathcal{L}_{n}(\mu)\right] } & =\mathfrak{u}_{-w_{0} \lambda-2 \rho^{\prime}+2 \rho_{\mathrm{l}},-w_{0} \mu-2 \rho^{\prime}+2 \rho_{\mathrm{l}}}(1) .
\end{aligned}
$$

Proof. The map $i$ is certainly a vector space isomorphism. One checks that the action of the translation functors on the parabolic Verma modules is compatible with the action of the divided powers of the Chevalley generators of $\mathfrak{U}_{q=1}$ on the monomial basis. Thus (1) and (2) follow. Now (3) follows from Theorem 4.14 and the definition of KL polynomials $\mathfrak{u}_{\mu, \nu}$ and $\mathfrak{l}_{\mu, \nu}$. 


\section{$\S 4.5$. The case as $n \mapsto \infty$}

By studying truncation functors $\operatorname{Tr}$ for $\mathcal{O}_{\mathbf{m}+n}^{+}$with varying $n$, analogous to Subsection 3.2 (cf. [Don]), we can establish the counterparts of Subsection 3.5.

The following theorem should be compared to Theorem 3.14. Note that Corollary 4.13 is used in proving (4) below.

Theorem 4.16. Let $\lambda \in X_{\mathbf{m}+\infty}^{+}$.

(1) There exists a unique tilting module $\mathcal{U}(\lambda)$ in $\mathcal{O}_{\mathbf{m}+\infty}^{++}$with $\mathcal{K}(\lambda)$ sitting at the bottom of a Verma flag. Moreover, $\mathcal{U}(\lambda)=\cup_{n} \mathcal{U}_{n}(\lambda)$.

(2) The functor $t_{n}$ sends $\mathcal{U}(\lambda)$ to $\mathcal{U}_{n}(\lambda)$ if $\left(\lambda \mid \delta_{n+1}\right)_{c}=0$ and to 0 otherwise.

(3) We have $(\mathcal{U}(\lambda): \mathcal{K}(\mu))=\left(\mathcal{U}_{n}(\lambda): \mathcal{K}_{n}(\mu)\right)$ for $n \gg 0$.

(4) The Verma flag lengths for $\mathcal{U}(\lambda)$ and $\mathcal{U}_{n}(\lambda)$ for $n \gg 0$ are the same (and finite).

The following proposition follows from Theorem 4.14, Corollary 4.13, and the properties of the truncation maps/functors.

Proposition 4.17. Let $n>n_{0}$ and $\lambda \in X_{\mathbf{m}+n_{0}}^{++}$. Extend $\lambda$ to $\lambda^{(n)} \in$ $X_{\mathbf{m}+n}^{++}$by letting $\lambda^{(n)}(i)=0$ for $n_{0}<i \leq n$. Then, there exists $n_{\lambda} \gg 0$ such that the Verma flag structure of $\mathcal{U}_{\lambda^{(n)}}$ is independent of $n \geq n_{\lambda}$.

\section{§4.6. A general super duality conjecture}

Based on Conjecture 3.10, Theorems 4.15 and 4.7 we propose the following conjecture which generalizes [CWZ, Conjecture 6.10], which will be referred to as the general super duality conjecture.

Conjecture 4.18. For a tuple of positive integers $\mathbf{m}$, the categories $\mathcal{O}_{\mathbf{m} \mid \infty}^{++}$and $\mathcal{O}_{\mathbf{m}+\infty}^{++}$are equivalent.

Remark 4.19. We regard Conjecture 4.18 as a pointer toward a profound connection between representation theories of Lie algebras and Lie superalgebras. One should keep in mind some variations of the conjecture such as an isomorphism of the full subcategories of modules with Verma flags, or an equivalence of derived categories, et cetera.

The validity of Conjecture 4.18 implies the validity of the parabolic Brundan-Kazhdan-Lusztig Conjecture 3.10, by using the Kazhdan-Lusztig theory for $\mathfrak{g l}(m+n)$ as formulated in Theorem 4.15 and the properties of truncation maps/functors (see Corollary 2.6 and Proposition 3.12). In particular, 
the original Brundan conjecture for the full category $\mathcal{O}$ of $\mathfrak{g l}(m \mid 1)$-modules (cf. Remark 3.11) would follow from the super duality conjecture.

\section{$\S 5$. Application of the Chuang-Rouquier $\mathfrak{s l}_{2}$-Categorification}

\section{$\S 5.1$. The $\mathfrak{s l}_{2}$-categorification and category $\mathcal{O}_{\mathbf{m} \mid n}^{+}$}

This subsection is a super analogue of Chuang-Rouquier [CR, 7.4].

Let $\left\{u_{i}\right\}$ be a $\mathbb{Z}_{2}$-homogeneous basis of $\mathfrak{g}=\mathfrak{g l}(m \mid n)$, and $\left\{u^{i}\right\}$ be its dual basis with respect to the supersymmetric bilinear form $\langle a, b\rangle:=\operatorname{str}(a b)$, where $a b$ denotes the matrix multiplication of $a, b \in \mathfrak{g l}(m \mid n)$. The Casimir $C:=$ $\sum_{i}(-1)^{\left|u_{i}\right|} u_{i} u^{i}$ lies in the center of the enveloping algebra $U(\mathfrak{g})$. By means of the standard matrix elements, we readily see that

$$
C=\sum_{i, j \in I(m \mid n)}(-1)^{\bar{j}} e_{i j} e_{j i} .
$$

Recall that $\bar{j}=0$ if $j<0$ and $\bar{j}=1$ if $j>0$. Denote by $\left\{x_{i}\right\}_{i \in I(m \mid n)}$ the standard basis for the natural $\mathfrak{g}$-module $V$, and set $\left|x_{i}\right|=\bar{i}$.

Given a $\mathfrak{g}$-module $M$, we let $X_{M} \in \operatorname{End}_{\mathfrak{g}}(V \otimes M)$ the adjoint map associated to the action map $\mathfrak{g} \times M \rightarrow M$ (by identifying $\mathfrak{g}=\operatorname{End}(V)$ ). It follows that

$$
X(v \otimes m)=\Omega(v \otimes m),
$$

where

$$
\Omega=\sum_{i, j \in I(m \mid n)}(-1)^{\bar{j}} e_{i j} \otimes e_{j i} .
$$

This defines an endomorphism $X$ of the functor $V \otimes-$. One verifies that (with all the superalgebra signs cancelling)

$$
\Omega=\frac{1}{2}\left(\Delta_{\mathfrak{g}}(C)-C \otimes 1-1 \otimes C\right),
$$

where $\Delta_{\mathfrak{g}}$ denotes the coproduct on $U(\mathfrak{g})$. We also define

$$
T_{M} \in \operatorname{End}_{\mathfrak{g}}(V \otimes V \otimes M), \quad v \otimes v^{\prime} \otimes m \mapsto(-1)^{|v|\left|v^{\prime}\right|} v^{\prime} \otimes v \otimes m .
$$

This defines an endomorphism $T$ of the functor $V \otimes V \otimes-$. 
Recall that the degenerate affine Hecke algebra $H_{\ell}$ is an algebra generated by $X_{i}(i=1, \ldots, \ell)$ and $s_{i}(i=1, \ldots, \ell-1)$, subject to the following relations:

$$
\begin{aligned}
s_{i}^{2}=1, \quad s_{i} s_{i+1} s_{i} & =s_{i+1} s_{i} s_{i+1}, \\
s_{i} s_{j} & =s_{j} s_{i}, \quad|i-j|>1, \\
x_{j} s_{i} & =s_{i} x_{j}, \quad(j \neq i, i+1), \\
x_{i+1} s_{i}-s_{i} x_{i} & =1, \\
x_{i} x_{j} & =x_{j} x_{i}, \quad(i \neq j) .
\end{aligned}
$$

The following is a super generalization of a theorem of Arakawa-Suzuki $[\mathrm{AS}]$.

Proposition 5.1. $\quad$ There is an algebra homomorphism

$$
\begin{aligned}
H_{\ell} & \longrightarrow \operatorname{End}_{\mathfrak{g}}\left(V^{\otimes \ell} \otimes M\right), \\
s_{i} & \mapsto \mathbf{1}_{V}^{\otimes \ell-i} \otimes T_{V}^{\otimes i-1} \otimes M,
\end{aligned}
$$

Proof. All the relations are straightforward to check except (5.2). The relation (5.2) is equivalent to the following identity in $\operatorname{End}_{\mathfrak{g}}(V \otimes V \otimes M)$ for $\mathfrak{g}$-module $M$ :

$$
T_{M} \circ\left(\mathbf{1}_{V} \otimes X_{M}\right)=X_{V \otimes M} \circ T_{M}-\mathbf{1}_{V \otimes V \otimes M} .
$$

Indeed, given $a, b \in I(m \mid n)$, we calculate that

$$
\begin{aligned}
X_{V \otimes M} T_{M}\left(x_{a} \otimes\right. & \left.x_{b} \otimes m\right)=(-1)^{\bar{a} \bar{b}} X_{V \otimes M}\left(x_{b} \otimes x_{a} \otimes m\right) \\
= & \sum_{i, j \in I(m \mid n)}(-1)^{\bar{a} \bar{b}+\bar{j}+(\bar{i}+\bar{j}) \bar{b}} e_{i j} x_{b} \otimes e_{j i}\left(x_{a} \otimes m\right) \\
= & \sum_{i, j \in I(m \mid n)}(-1)^{\bar{a} \bar{b}+\bar{j}+(\bar{i}+\bar{j}) \bar{b}} e_{i j} x_{b} \otimes e_{j i} x_{a} \otimes m \\
& +\sum_{i, j \in I(m \mid n)}(-1)^{\bar{a} \bar{b}+\bar{j}+(\bar{i}+\bar{j})(\bar{b}+\bar{a})} e_{i j} x_{b} \otimes x_{a} \otimes e_{j i} m \\
& =x_{a} \otimes x_{b} \otimes m+T_{M} \sum_{i, j \in I(m \mid n)}(-1)^{\bar{j}+(\bar{i}+\bar{j}) \bar{b}} x_{a} \otimes e_{i j} x_{b} \otimes e_{j i} m \\
& =\left(\mathbf{1}_{V \otimes V \otimes M}+T_{M} \circ\left(\mathbf{1}_{V} \otimes X_{M}\right)\right)\left(x_{a} \otimes x_{b} \otimes m\right) .
\end{aligned}
$$

We write $\lambda \rightarrow_{a} \mu$ if there exists $i \in I(m \mid 0)$ such that $\lambda_{i}-i=a, \mu_{i}-i=a+1$, or if there exists $i \in I(0 \mid n)$ such that $-\lambda_{i}+i=a+1,-\mu_{i}+i=a$, and in addition, 
$\lambda_{j}=\mu_{j}$, for all $j \neq i$. Given two (integral) blocks $\mathcal{O}_{\gamma}^{+}, \mathcal{O}_{\gamma^{\prime}}^{+}$in the category $\mathcal{O}_{\mathbf{m} \mid n}^{+}$ corresponding to $\gamma, \gamma^{\prime} \in P$, we write $\gamma \rightarrow_{a} \gamma^{\prime}$ if there exists $\lambda, \mu \in \mathfrak{h}^{*}$ such that $K_{n}(\lambda) \in \mathcal{O}_{\gamma}^{+}$and $K_{n}(\mu) \in \mathcal{O}_{\gamma^{\prime}}^{+}$. Denote by $\operatorname{pr}_{\gamma}$ the projection onto the block $\mathcal{O}_{\gamma}^{+}$. We can rewrite the translation functors $F_{a}(3.3)$ as

$$
F_{a}=\bigoplus_{\gamma, \gamma^{\prime}: \gamma \rightarrow a \gamma^{\prime}} \operatorname{pr}_{\gamma^{\prime}} \circ(V \otimes-) \circ \operatorname{pr}_{\gamma}
$$

Proposition 5.2. $\quad$ The translation functor $F_{a}$ can be identified with the generalized $(a-m)$-eigenspace of $X$ acting on $V \otimes-$.

Proof. It suffices to check the proposition on a parabolic Verma module $K_{n}(\lambda)$. The Casimir acts on $K_{n}(\lambda)$ as the scalar multiplication by $c_{\lambda}:=\langle\lambda+$ $2 \rho, \lambda\rangle$. By (5.1), $\Omega$ acts on a subquotient $K_{n}\left(\lambda+\delta_{i}\right)$ in $V \otimes K_{n}(\lambda)$ (where we recall $\left.V=L_{n}\left(\delta_{-m}\right)\right)$ as the multiplication by

$$
\begin{aligned}
\frac{1}{2}\left(c_{\lambda+\delta_{i}}-c_{\lambda}-c_{\delta_{-m}}\right) \\
\quad=\frac{1}{2}\left(\left\langle\lambda+\delta_{i}+2 \rho, \lambda+\delta_{i}\right\rangle-\langle\lambda+2 \rho, \lambda\rangle-\left\langle\delta_{-m}+2 \rho, \delta_{-m}\right\rangle\right) \\
=\left\langle\lambda, \delta_{i}\right\rangle+\frac{1}{2}\left\langle\delta_{i}, \delta_{i}\right\rangle-\frac{1}{2}\left\langle\delta_{-m}, \delta_{-m}\right\rangle-\left\langle\rho, \delta_{-m}-\delta_{i}\right\rangle \\
\quad= \begin{cases}\lambda_{i}-i-m, & \text { if } i \in I(m \mid 0) \\
-\lambda_{i}+i-m-1, & \text { if } i \in I(0 \mid n) .\end{cases}
\end{aligned}
$$

The statement now follows by comparing with the definition of $F_{a}$.

We can identify $E_{a}$ similarly. Note that the notations $E$ and $F$ are switched in [CR]. Following [CR, 7.4], Propositions 5.1 and 5.2 above imply that $E_{a}, F_{a}, X, T$ satisfy the definition of the $\mathfrak{s l}_{2}$-categorification (which we will skip here and refer to [CR, 5.1.1, 5.2.1] for detail).

\section{§5.2. A formal consequence}

By definition, the (divided power) translation functors $E_{a}^{(i)}, F_{a}^{(i)}$ for $i \geq$ 1 , are obtained from the functors $E^{i}, F^{i}$ by replacing $V^{\otimes i}$ by the symmetric products $S^{i} V^{*}, S^{i} V$ et cetera).

We shall need the following formal consequence of the $\mathfrak{s l}_{2}$-categorification (see [CR, Proposition 5.23] and a statement in its proof).

Theorem 5.3. For every simple object $L$ in $\mathcal{O}_{\mathbf{m} \mid n}^{+}, i \leq d:=\max \left\{j \mid F^{j}(L)\right.$ $\neq 0\}$, and $a \in \mathbb{Z}$, the socle and cosocle of $F^{(i)} L$ are simple and isomorphic. Furthermore, $F^{(d)} L$ is simple. 


\section{§6. Some Results on Canonical Basis and Tilting Modules}

In this section we establish some miscellaneous results on canonical basis elements and tilting modules that will be used in subsequent sections.

\section{$\S 6.1$. The L operators}

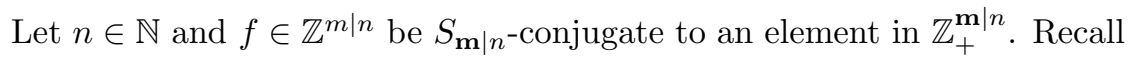
that $m=m_{1}+\cdots+m_{s}$. Let $-m \leq i<0<j \leq n$ with $f(i)=f(j)$. We define the L operators (cf. [Br1])

$$
\mathrm{L}_{i, j}(f):=f-a\left(d_{i}-d_{j}\right),
$$

where $a$ is the smallest positive integer such that $f-a\left(d_{i}-d_{j}\right)$ and all $\mathrm{L}_{k, l}(f)-$ $a\left(d_{i}-d_{j}\right)$ for $-m \leq i, k<0<l<j \leq n$ with $f(k)=f(l)$ are $S_{\mathbf{m} \mid n}$-conjugate to elements of $\mathbb{Z}_{+}^{\mathbf{m} \mid n}$.

Now let $f \in \mathbb{Z}_{+}^{\mathbf{m} \mid n}$ and suppose $\# f=k$. Let $-m \leq i_{i}, i_{2}, \ldots, i_{k} \leq-1$ and $1 \leq j_{k}<j_{k-1}<\cdots<j_{1} \leq n$ be such that $f\left(i_{l}\right)=f\left(j_{l}\right)$, for $l=1, \ldots, k$. For a $k$-tuple $\theta=\left(\theta_{1}, \ldots, \theta_{k}\right) \in \mathbb{N}^{k}$ we define $[\operatorname{Br} 1]$

$$
f^{\mathrm{L} \theta} \equiv \mathrm{L}_{\theta}(f)=\left(\mathrm{L}_{i_{k}, j_{k}}^{\theta_{k}} \circ \cdots \circ \mathrm{L}_{i_{1}, j_{1}}^{\theta_{1}}(f)\right)^{+},
$$

where the superscript + here stands for the unique $S_{\mathbf{m} \mid n}$-conjugate in $\mathbb{Z}_{+}^{\mathbf{m} \mid n}$.

\section{§6.2. The positive pairs}

In this subsection we set $\mathbf{m}=\left(m_{1}, m_{2}\right)$ with $m_{1}+m_{2}=m$, and shall adapt here the notion of positive pairs defined in $\mathbb{Z}_{+}^{m_{1}, m_{2} \mid 0}$ from [CWZ].

Let $f \in \mathbb{Z}_{+}^{m_{1}, m_{2} \mid n}$. For a pair of integers $(i \mid j)$ such that $-m \leq i<-m_{2} \leq$ $j<0$, we define the distance of $(i \mid j)$ (associated to $f \in \mathbb{Z}_{+}^{\mathbf{m}+n}$ ) to be $d(i \mid j):=$ $f(i)-f(j)$. We call $(i \mid j)$ an admissible pair for $f$ if $f(i)>f(j)$ and $f \cdot \tau_{i j}$ affords a (unique) conjugate $\left(f \cdot \tau_{i j}\right)^{+} \in \mathbb{Z}_{+}^{\mathbf{m} \mid n}$. Two admissible pairs $\left(i_{1} \mid j_{1}\right)$ and $\left(i_{2} \mid j_{2}\right)$ for $f$ are said to be disjoint, if $i_{1} \neq i_{2}$ and $j_{1} \neq j_{2}$. Two subsets $A_{1}$ and $A_{2}$ of admissible pairs of $f$ are said to be disjoint, if any two admissible pairs $\left(i_{1} \mid j_{1}\right) \in A_{1}$ and $\left(i_{2} \mid j_{2}\right) \in A_{2}$ are disjoint. Let $A_{f}^{+}$denote the set of all admissible pairs of $f$. For $k \geq 1$ we define recursively $\Sigma_{f}^{k}:=\{(i \mid j) \in$ $A_{f}^{+} \mid d(i \mid j)=k$ and $(i \mid j)$ disjoint from $\left.\sqcup_{s=1}^{k-1} \Sigma_{f}^{s}\right\}$. Let $\Sigma^{+}(f) \equiv \Sigma_{f}^{+}:=\sqcup_{k \geq 1} \Sigma_{f}^{k}$. An element in $\Sigma_{f}^{+}$is called a positive pair of $f$. Given a subset $\Sigma$ of positive pairs of $f$, we denote by $f_{\Sigma}$ the element in $\mathbb{Z}_{+}^{\mathbf{m} \mid n}$ obtained by first interchanging 
the values of $f$ at each positive pair in $\Sigma$, and then taking the unique $S_{\mathbf{m} \mid n^{-}}$ conjugate in $\mathbb{Z}_{+}^{\mathbf{m} \mid n}$.

Set $I(m \mid n)=I_{1} \sqcup I_{2} \sqcup I_{3}$, where $I_{1}, I_{2}, I_{3}$ are the increasing subintervals of $I(m \mid n)$ of length $m_{1}, m_{2}, n$ respectively. We denote by $f_{a b}$ the restriction of $f$ to $I_{a} \cup I_{b}$ with $a \leq b$ and let $f_{a}=f_{a a}$.

\section{$\S 6.3$. On tilting modules in $\mathcal{O}_{m_{1}, m_{2} \mid n}^{+}$}

Given $\lambda \in X_{\mathbf{m} \mid n}^{+}$, by abuse of notation we denote $U_{n}\left(f_{\lambda}\right)=U_{n}(\lambda), K_{n}\left(f_{\lambda}\right)$ $=K_{n}(\lambda)$ and $L_{n}\left(f_{\lambda}\right)=L_{n}(\lambda)$, the respective tilting, parabolic Verma and irreducible $\mathfrak{g l}(m \mid n)$-modules.

Let $f \in \mathbb{Z}_{+}^{m_{1}, m_{2} \mid n}$ with $m=m_{1}+m_{2}$. We denote by $K^{12}\left(f_{12}\right)$ the parabolic Verma $\mathfrak{g l}\left(m_{1}+m_{2}\right)$-module. Likewise the notation $K^{23}\left(f_{23}\right)$ denotes the parabolic Verma $\mathfrak{g l}\left(m_{2} \mid n\right)$-module.

Proposition 6.1. Let $f \in \mathbb{Z}_{+}^{m_{1}, m_{2} \mid n}$.

(i) Let $\Sigma$ be a positive pair of $f$. Assume that $\operatorname{Ext}^{1}\left(K^{12}\left(\left(f_{\Sigma}\right)_{12}\right), K^{12}\left(f_{12}\right)\right) \neq$

0 . Suppose that for any $g \prec f$ with $\left.\left(U_{n}(f)\right): K_{n}(g)\right) \neq 0$ we have $g \nsucc f_{\Sigma}$. Then $\left(U_{n}(f): K_{n}\left(f_{\Sigma}\right)\right) \geq 1$.

(ii) Let $-m_{2} \leq i<0<j \leq n$ be such that $f(i)=f(j)$. Assume that $\operatorname{Ext}^{1}\left(K^{23}\left(f_{23}^{L_{i, j}}\right), K^{23}\left(f_{23}\right)\right) \neq 0$. Suppose that for any $g \prec f$ with $\left(U_{n}(f)\right)$ : $\left.K_{n}(g)\right) \neq 0$ we have $g \nsucc f_{23}^{L_{i, j}}$. Then $\left(U_{n}(f): K_{n}\left(f_{23}^{L_{i, j}}\right)\right) \geq 1$.

Proof. We will only show (i). The proof of (ii) is similar.

Let $\Sigma=(i \mid j)$ be such that $-m \leq i<-m_{2} \leq j<0$ and $f(i)>f(j)$. Let $g=f_{\Sigma}$. Let $L_{0}^{12}\left(f_{12}\right)$ and $L_{0}^{12}\left(g_{12}\right)$ denote the irreducible $\mathfrak{p}_{m_{1}, m_{2}}$ modules of highest weights $f_{12}$ and $g_{12}$ respectively, where $\mathfrak{p}_{m_{1}, m_{2}}$ is the parabolic subalgebra of $\mathfrak{g l}\left(m_{1}+m_{2}\right)$ with Levi subalgebra $\mathfrak{g l}\left(m_{1}\right) \oplus \mathfrak{g l}\left(m_{2}\right)$. By assumption there exists a non-split extension $T$ of $\mathfrak{g l}\left(m_{1}+m_{2}\right)$-modules

$$
0 \longrightarrow \operatorname{Ind}_{\mathfrak{p}_{m_{1}, m_{2}}}^{\mathfrak{g l}\left(m_{1}+m_{2}\right)} L_{0}^{12}\left(f_{12}\right) \longrightarrow T \longrightarrow \operatorname{Ind}_{\mathfrak{p}_{m_{1}, m_{2}}}^{\mathfrak{g l}\left(m_{1}+m_{2}\right)} L_{0}^{12}\left(g_{12}\right) \longrightarrow 0 .
$$

Tensoring the above sequence with the simple $\mathfrak{g l}(n)$-module $L^{3}\left(f_{3}\right)=L^{3}\left(g_{3}\right)$, we obtain a non-split extension of $\mathfrak{g l}\left(m_{1}+m_{2}\right) \oplus \mathfrak{g l}(n)$-modules

$$
0 \rightarrow \operatorname{Ind}_{\mathfrak{p}_{m_{1}, m_{2}}}^{\mathfrak{g l}\left(m_{1}+m_{2}\right)} L_{0}^{12}\left(f_{12}\right) \otimes L^{3}\left(f_{3}\right) \rightarrow T^{\prime} \rightarrow \operatorname{Ind}_{\mathfrak{p}_{m_{1}, m_{2}}}^{\mathfrak{g l}\left(m_{1}+m_{2}\right)} L_{0}^{12}\left(g_{12}\right) \otimes L^{3}\left(g_{3}\right) \rightarrow 0 .
$$

By applying an induction functor to (6.1), we obtain a short exact sequence of $\mathfrak{g l}\left(m_{1}+m_{2} \mid n\right)$-modules

$$
0 \rightarrow K_{n}(f) \rightarrow T^{\prime \prime} \rightarrow K_{n}(g) \rightarrow 0 .
$$


Taking the invariants of (6.2) with respect to the niradical of the parabolic $\mathfrak{p}$ whose Levi is $\mathfrak{g l}(m) \oplus \mathfrak{g l}(n)$ we recover (6.1), and hence the indecomposability of $T^{\prime \prime}$ follows from that of $T^{\prime}$.

Finally from the construction of tilting modules [So2, Br2] our second hypothesis above assures that $\left(U_{n}(f): K_{n}(g)\right) \geq 1$.

\section{§6.4. Tilting modules with short Verma flags}

We recall the following variant of [Br1, Corollary 4.27], which follows from the fact that $E_{a}^{(r)}, F_{a}^{(r)}$ are exact functors and are both left and right adjoint to each other.

Lemma 6.2. Let $U$ be a tilting module in $\mathcal{O}_{\mathbf{m} \mid n}^{+}$. Then $X_{a}^{(r)} U$ is a direct sum of tilting modules, where $X=E, F$.

Lemma 6.3. Let $\lambda \in X_{+}^{\mathbf{m} \mid n}$ be atypical. Then the parabolic Verma module $K_{n}(\lambda)$ is not a tilting module.

Proof. For atypical $\lambda, K_{n}(\lambda)$ is reducible since the Kac module (which is the parabolic Verma module with respect to the parabolic subalgebra whose Levi subalgebra is $\mathfrak{g l}(m) \oplus \mathfrak{g l}(n))$ as its quotient is reducible. If $K_{n}(\lambda)$ were a tilting module, then by Coroallry 3.8 we have $K_{n}(\lambda)=K_{n}(\lambda)^{\tau}$. But this is impossible since $K_{n}(\lambda)$ is reducible and hence cannot have isomorphic socle and cococle.

Proposition 6.4. Let $h, f \in \mathbb{Z}_{+}^{\mathbf{m} \mid n}$ be atypical. Suppose that (i) $U_{f}=$ $X U_{h}$ for a product $X$ of $E_{a}^{(r)}$ and $F_{a}^{(r)}$ with varying a and $r$, (ii) $i\left[U_{n}(h)\right]=$ $U_{h}(1)$, and (iii) $U_{f}$ has at most three monomial terms. Then $X U_{n}(h)=U_{n}(f)$, and $i\left[U_{n}(f)\right]=U_{f}(1)$.

Proof. By Theorem 3.9 and the assumptions (i-ii), we have that $i\left[X U_{n}(h)\right]$ $=X i\left[U_{n}(h)\right]=X U_{h}(1)=U_{f}(1)$. It follows from this and Lemma 6.2 that there is a summand of $X U_{n}(h)$ isomorphic to $U_{n}(f)$. So the proposition follows by showing that $X U_{n}(h)$ is indecomposable. By the assumption (iii) and Theorem 3.9, $X U_{n}(h)$ has a Verma flag of length at most three. The weight $g$ in any Verma $K_{n}(g)$ appearing in a Verma flag of $X U_{n}(h)$ must be atypical like $f$. Thus $K_{n}(g)$ is not tilting by Lemma 6.3 , and hence $X U_{n}(h)$ has to be indecomposable. 


\section{$\S 6.5$. The typical case}

The next proposition is a variant of [Br1, Lemma 2.25] and [Br1, Theorem 4.31]. It can be proved by modifying the arguments therein, using now Theorem 4.15 .

Proposition 6.5. Let $f \in \mathbb{Z}_{+}^{\mathbf{m}+n}$ be typical and let $f_{\mathbf{m}}$ denote the restriction of $f$ to the set $I(m \mid 0)$. We have

(i) $U_{f}=U_{f_{\mathrm{m}}} \otimes w_{f(1)} \wedge \cdots \wedge w_{f(n)}$, where $U_{f_{\mathbf{m}}}$ is the corresponding canonical basis element in $\mathcal{E}^{\mathbf{m}+0}$.

(ii) The linear map $i$ sends $\left[U_{n}(f)\right]$ to $U_{f}(1)$.

\section{$\S 6.6$. The regular case}

We introduce a Regularity Condition (R) on $f \in \mathbb{Z}_{+}^{m_{1}, m_{2} \mid n}$ :

(R) If $f(i)=f(j)=a$ for some $a \in \mathbb{Z}$ and $-m_{1}-m_{2} \leq i<0<j$, then there exists no $k \in I\left(m_{1}+m_{2} \mid n\right) \backslash\{i, j\}$ with $f(k)=a-1$ or $f(k)=a$.

Theorem 6.6. $\quad$ Suppose that $f \in \mathbb{Z}_{+}^{m_{1}, m_{2} \mid n}$ satisfies Condition $(R)$. Then we have

(i) $U_{f}=\sum_{\theta \in\{0,1\} \# f} \sum_{\Sigma \subseteq \Sigma^{+}(f)} q^{|\theta|+|\Sigma|} K_{f_{\Sigma}^{L_{\theta}}}$ in $\widehat{\mathcal{E}}^{m_{1}, m_{2} \mid n}$,

(ii) $\left[U_{n}(f)\right]=\sum_{\theta \in\{0,1\} \# f} \sum_{\Sigma \subseteq \Sigma^{+}(f)}\left[K_{n}\left(f_{\Sigma}^{L_{\theta}}\right)\right]$ in $G\left(\mathcal{O}_{m_{1}, m_{2} \mid n}^{+}\right)$,

(iii) the tilting module $U_{n}(f)$ is $\tau$-self-dual and it has a simple cosocle $L_{n}(\tilde{f})$, where $\tilde{f}=f_{\Sigma^{+}(f)}^{L_{(1, \ldots, 1)}}$ is the minimal weight in a Verma flag of $U_{n}(f)$.

In particular, a Verma flag of $U_{n}(f)$ is multiplicity-free and has length $2^{\left|\Sigma^{+}(f)\right|+\# f}$.

Proof. Let $\# f=k$ and $\left\{\left(i_{1} \mid j_{1}\right), \cdots,\left(i_{k} \mid j_{k}\right)\right\}$ be the set of all pairs of $f$ with $f\left(i_{t}\right)=f\left(j_{t}\right)$ for $1 \leq t \leq k$, where $0<j_{1}<\cdots<j_{k}$. Since $f$ satisfies $(\mathrm{R})$, we have $\Sigma^{+}\left(f^{\mathrm{L}_{\theta}}\right)=\Sigma^{+}(f)$ for every $\theta \in\{0,1\} \# f$, and moreover, $\mathrm{L}_{i_{s}, j_{s}} \circ \mathrm{L}_{i_{t}, j_{t}}=\mathrm{L}_{i_{t}, j_{t}} \circ \mathrm{L}_{i_{s}, j_{s}}$.

Take $\left(i_{k} \mid j_{k}\right)$ with $f\left(i_{k}\right)=f\left(j_{k}\right)=a_{k}$. Assume without loss of generality that $f$ is of the form

$$
\left(\cdots a_{k} \cdots|\cdots| \cdots a_{k} \cdots\right) \text {. }
$$

(We omit the parallel proof when $f$ is of the form $\left(\cdots\left|\cdots a_{k} \cdots\right| \cdots a_{k} \cdots\right)$ ) 
We prove (i) by induction on the atypicality number \# $f$. By Proposition 6.5 , the case $\# f=0$ boils down to [CWZ, Theorem 4.25].

Let $h$ be defined by $h\left(j_{k}\right)=a_{k}-1$ and $h(s)=f(s)$, for all $s \neq j_{k}$. Note that $\# h=\# f-1$ and $\left|\Sigma^{+}(h)\right|=\left|\Sigma^{+}(f)\right|$, and the induction assumption gives an explicit formula for $U_{h}$ in $2^{\left|\Sigma^{+}(f)\right|+\# f-1}$ monomial terms. Set $X=E_{a_{k}-1}$. Then $X U_{h}$ is clearly bar-invariant and by a direct calculation is equal to the right-hand side in (i), hence it has to coincide with $U_{f}$ by definition of canonical basis. This proves (i).

We prove (ii) and (iii) together in two inductive steps: (1) induction on the atypicality number $\# f$ to reduce to the case when $\# f=0 ;(2)$ in the case when $\# f=0$, induction on the cardinality $\left|\Sigma^{+}(f)\right|$. In the initial case when $\# f=\left|\Sigma^{+}(f)\right|=0, f$ is minimal in super Bruhat ordering and $K_{n}(f)$ is irreducible, and hence $U_{n}(f)=K_{n}(f)$ has a simple cosocle. The arguments (which are based on Method One of the proof of [Br1, Theorem 4.37]) for these two steps are completely analogous, and we will only present the inductive step (1) on \#f in detail below.

By $(\mathrm{R})$, for each $g \preceq f$ we clearly have $F_{a_{k}-1}^{2} K_{g}=0$, hence $F_{a_{k}-1}^{2} K_{n}(g)=$ 0 and then $F_{a_{k}-1}^{2} L_{n}(g)=0$. It follows by Theorem 5.3 that $F_{a_{k}-1} L_{n}(g)$ is irreducible or zero depending on whether or not $F_{a_{k}-1} K_{g}$ is zero (or equivalently, depending on whether $\left(a_{k}-1\right)$-string of the underlying crystal graph has length 1 or 0$)$. Suppose that

$$
\operatorname{Hom}_{\mathbf{m} \mid n}\left(X U_{n}(h), L_{n}(g)\right) \cong \operatorname{Hom}_{\mathbf{m} \mid n}\left(U_{n}(h), F_{a_{k}-1} L_{n}(g)\right)
$$

is nonzero for some $g \preceq f$. By the inductive assumption, the tilting module $U_{n}(h)$ has a simple cosocle $L_{n}(\tilde{h})$. Thus, $F_{a_{k}-1} L_{n}(g)=L_{n}(\tilde{h})$ by Theorem 5.3. Hence $\tilde{F}_{a_{k}-1} g=\tilde{h}$, and thus $g=\tilde{E}_{a_{k}-1} \tilde{h}$ since the $\left(a_{k}-1\right)$-string of the underlying crystal graph is of length 1 , where $\tilde{E}_{a_{k}-1}, \tilde{F}_{a_{k}-1}$ denote the Kashiwara (crystal) operators corresponding to $E_{a_{k}-1}, F_{a_{k}-1}$. One checks that $\tilde{E}_{a_{k}-1} \tilde{h}=\tilde{f}$. Hence $X U_{n}(h)$ has a simple cosocle $L_{n}(\tilde{f})$ and in particular is indecomposable. This proves (iii).

Now by the induction assumption and Theorem 3.9, we have

$$
i\left[X U_{n}(h)\right]=X i\left[U_{n}(h)\right]=X U_{h}(1)=U_{f}(1) .
$$

It follows by Lemma 6.2 and the indecomposability of $X U_{n}(h)$ that $U_{n}(f)=$ $X U_{n}(h)$. Together with (i), this proves (ii).

Remark 6.7. Setting $n=0$, the proof of Theorem 6.6 gives a purely algebraic proof of the Kazhdan-Lusztig conjecture for the parabolic category $\mathcal{O}_{+}^{m_{1}, m_{2}}$ of $\mathfrak{g l}\left(m_{1}+m_{2}\right)$-modules (compare with Theorem 4.14). 
Remark 6.8. Recall from Remark 2.4 that $\mathbb{Z}_{+}^{m_{1}, m_{2} \mid n_{1}, n_{2}}$ parameterizes the bases for the space $\mathcal{E}^{m_{1}, m_{2} \mid n_{1}, n_{2}}$. Suppose that $f$ satisfies the following condition:

(RR) If $f(i)=f(j)=a$ for some $a \in \mathbb{Z}$ with $i<0<j$, then there exists no $k \in I\left(m_{1}+m_{2} \mid n_{1}+n_{2}\right) \backslash\{i, j\}$ with $f(k)=a-1$ or $f(k)=a$.

Denote by $\Sigma^{+}\left(-f_{34}\right)$ the set of positive pairs of $-f_{34}$. Since $f$ satisfies Condition (RR), we have $\Sigma^{+}\left(f_{12}\right)=\Sigma^{+}\left(f_{12}^{\mathrm{L}_{\theta}}\right)$ and $\left.\Sigma^{+}\left(-f_{34}\right)=\Sigma^{+}\left(-f_{34}^{\mathrm{L}_{\theta}}\right)\right)$, for any $\theta \in$ $\{0,1\}^{\# f}$. The argument for Theorem 6.6 can be modified easily to establish the following formula for the canonical basis:

$$
U_{f}=\sum_{\theta \in\{0,1\}^{\# f}} \sum_{\Sigma \subseteq \Sigma^{+}\left(f_{12}^{\mathrm{L}}\right)} \sum_{\Gamma \subseteq \Sigma^{+}\left(-f_{34}^{\mathrm{L}}\right)} q^{|\theta|+|\Sigma|+|\Gamma|} K_{f_{\Sigma, \Gamma}^{\mathrm{L} \theta}} .
$$

Here $f_{\Sigma, \Gamma}^{\mathrm{L}_{\theta}}$ denotes the function obtained from $f^{\mathrm{L}_{\theta}}$ by first interchanging the values of $f$ at each positive pair in $\Sigma$ and $\Gamma$, and then taking the unique conjugate under $S_{m_{1}} \times S_{m_{2}} \times S_{n_{1}} \times S_{n_{2}}$ in $\mathbb{Z}_{+}^{m_{1}, m_{2} \mid n_{1}, n_{2}}$.

The corresponding multiplicity-free formula holds for the tilting module in the category $\mathcal{O}_{+}^{m_{1}, m_{2} \mid n_{1}, n_{2}}$ (see Remark 3.11).

Remark 6.9. For $f$ satisfying the condition $(\mathrm{R})$ or $(\mathrm{RR})$, the formulae for $U_{f}$ and $U_{n}(f)$ above support Conjecture 3.10 .

\section{$\S 7$. The Category $\mathcal{O}_{1,1 \mid n}^{+}$of $\mathfrak{g l}(2 \mid n)$-Modules}

In this section, we analyze completely the case for $\mathbf{m}=(1,1)$. We find explicit formulas for canonical basis in $\mathcal{E}^{1,1 \mid n}$, and establish the parabolic Brundan Conjecture 3.10 for the category $\mathcal{O}_{1,1 \mid n}^{+}$.

\section{§7.1. A procedure for canonical basis}

For $f \in \mathbb{Z}_{+}^{1,1 \mid n}$, we denote $\Sigma_{f_{13}}=(-2 \mid j)$ if there exists $j>0$ with $f(-2)=$ $f(j)$, and otherwise set $\Sigma_{f_{13}}=\emptyset$. Similarly, denote $\Sigma_{f_{23}}=(-1 \mid j)$ if there exists $j>0$ with $f(-1)=f(j)$, and otherwise set $\Sigma_{f_{23}}=\emptyset$. If $\Sigma_{f_{13}} \cup \Sigma_{f_{23}}=\emptyset$, then $\# f=0$. Below we give a procedure to reduce any $f$ such that $\Sigma_{f_{13}} \cup \Sigma_{f_{23}} \neq \emptyset$ to $g$ such that $\Sigma_{g_{13}} \cup \Sigma_{g_{23}}=\emptyset$.

Procedure 7.1. Let $f \in \mathbb{Z}_{+}^{1+1 \mid n}$ be such that $\Sigma_{f_{13}} \cup \Sigma_{f_{23}} \neq \emptyset$.

Step 1 If $\Sigma_{f_{13}}=\emptyset$ go to Step 4. Otherwise go to Step 2 . 
Step 2 If $f(-2) \neq f(-1)$, go to Step 3. Otherwise let $h$ be the function obtained from $f$ by setting $h(-1)=h(-2)=f(-2)-1$ and $h(i)=f(i)$, for $i>0$. Let $X=F_{f(-2)-1}^{(2)}$. Stop.

Step 3 Let $h$ be the function obtained from $f$ by setting $h(-2)=f(-2)-1$ and $h(i)=f(i)$, for $i \neq-2$. Let $X=F_{f(-2)-1}$. Stop.

Step 4 If $f(-2)=f(-1)-1$ go to Step 5. Otherwise we let $h$ be the function defined by $h(-1)=f(-1)-1$ and $h(s)=f(s)$, for $s \neq-1$. Let $X=$ $F_{f(-1)-1}$. Stop.

Step 5 If there exists $i>0$ with $f(i)=f(-2)-1$, go to Step 6. Otherwise go to Step 3.

Step 6 Let $j>0$ with $f(-1)=f(j)$. Let $k>1$ be the smallest integer such that $f(j)-k \neq f(j-k+1)$. Let $h$ be defined by $h(j-k)=f(j-k)-1$ and $h(s)=f(s)$, for $s \neq j-k$. Let $X=F_{f(j-k)-1}$. Stop.

As can be seen case by case below, repeated application of the above procedure will produce in finite steps an element $g$ such that $\# g=0$.

Theorem 7.2. $\quad$ Let $f$ be such that $\Sigma_{f_{13}} \cup \Sigma_{f_{23}} \neq \emptyset$. Let $X$ and $h$ be as defined in Procedure 7.1. Then we have

(i) $U_{f}=X U_{h}$ in $\mathcal{E}^{1,1 \mid n}$,

(ii) $X U_{n}(h)=U_{n}(f)$ in $\mathcal{O}_{1,1 \mid n}^{+}$,

(iii) $i\left[U_{n}(f)\right]=U_{f}(1)$,

(iv) the tilting module $U_{n}(f)$ is $\tau$-self-dual and it has a simple cosocle.

The proof of Theorem 7.2 will be postponed to the following subsections. We note the following immediate consequence.

Theorem 7.3. $\quad$ The Conjecture 3.10 for the category $\mathcal{O}_{1,1 \mid n}^{+}$holds.

Proof. The case of typical weights is taken care by Proposition 6.5. The case of atypical weights follows from Theorem 7.2.

Below as usual we will denote by $\cdots$ an expression with no $a$ or $a-1$. The proof for Theorem 7.2 is done case by case, and the main argument in most cases is the same as the one for Theorem 6.6. In particular, a main point of the argument is to check if the assumption in Theorem 5.3 is also satisfied. 


\section{§7.2. Proof of Theorem 7.2, I}

In the subsection, we consider the case when $\left|\Sigma_{f_{13}}\right|=1$. Here we have the following possibilities:

(i) $f=(a|a| \cdots a \cdots)$

(ii) $f=(a|a| \cdots a-1, a \cdots)$

(iii) $f=(a|a-1| \cdots a-1, a \cdots)$

(iv) $f=(a|\cdots| \cdots a \cdots)$

(v) $f=(a|a-1| \cdots a \cdots)$

(vi) $f=(a|\cdots| \cdots a-1, a \cdots)$

In (i) we set $h=(a-1|a-1| \cdots a \cdots)$ and $X=F_{a-1}^{(2)}$. We note that $h$ is a typical weight and hence we have $U_{(a-1|a-1| \cdots a \cdots)}=K_{(a-1|a-1| \cdots a \cdots)}$. Thus

$$
\begin{aligned}
U_{(a|a| \cdots a \cdots)} & =X U_{(a-1|a-1| \cdots a \cdots)} \\
& =K_{(a|a| \cdots a \cdots)}+q K_{(a|a-1| \cdots a-1 \cdots)}+q^{2} K_{(a|a| \cdots a-1 \cdots)} .
\end{aligned}
$$

It follows now from Proposition 6.4 that $U_{n}(f)=X U_{n}(h)$. Alternatively we can show this using the same type of argument as in the proof of Theorem 6.6 as follows. Suppose that $g \preceq f$ in the Bruhat ordering. Then $g$ must be of the form $(a|a| \cdots a \cdots),(a-1|a| \cdots a-1 \cdots),(a|a-1| \cdots a-1 \cdots),(\cdots|a| \cdots)$ or $(a|\cdots| \cdots)$. It is easy to see that $Y^{3} L_{n}(g)=0$, where $Y=E_{a-1}$. Thus in this case the assumptions of Theorem 5.3 is satisfied and hence $Y^{(2)} L_{n}(g)$ is irreducible. Therefore the same argument for Theorem 6.6 can be applied to show that $X U_{n}((a-1|a-1| \cdots a \cdots))$ has a simple cosocle and is isomorphic to $U_{n}((a|a| \cdots a \cdots))$.

In (ii) we set $h=(a-1|a-1| \cdots a-1, a \cdots)$ and $X=F_{a-1}^{(2)}$. Now any weight less than $h$ is of the form $(\cdots|a-1| \cdots a \cdots)$ or $(\cdots|a-1| \cdots a \cdots)$. Thus upon application of $X$ the $q$-power is preserved. It follows therefore that $X U_{(a-1|a-1| \cdots a-1, a \cdots)}=U_{(a|a| \cdots a-1, a \cdots)}$. Furthermore it is easy to check that if $g \preceq f$, then $Y^{3} g=0$, and hence the assumption in Theorem 5.3 is also satisfied. Thus $X U_{n}((a-1|a-1| \cdots a-1, a \cdots))=U_{n}((a|a| \cdots a-1, a \cdots))$.

In (iii) set $h=(a-1|a-1| \cdots a-1, a \cdots)$ and $X=F_{a-1}$. If $g \prec$ $h$, then $g$ is of the form $(\cdots|a-1| \cdots a \cdots)$ or $(a-1|\cdots| \cdots a \cdots)$ and so we see that $U_{f}=X U_{h}$. If $g \preceq f$, then $g$ is of the form $(a|a-1| \cdots a-$ $1, a \cdots),(a-1|a| \cdots a-1, a \cdots),(\cdots|a-1| \cdots a-1 \cdots),(a-1|\cdots| \cdots a-1 \cdots)$, 
$(\cdots|a| \cdots a \cdots)$ or $(a|\cdots| \cdots a \cdots)$. Let $Y=E_{a-1}$ and we see that $Y^{2} g=0$ satisfying the assumption of Theorem 5.3.

In (iv) set $h=(a-1|\cdots| \cdots a \cdots)$ and $X=F_{a-1}$. If $g \prec h$, then $g$ is of the form $(a-1|\cdots| \cdots a \cdots),(\cdots|a-1| \cdots a \cdots)$, or $(a-1|a-1| \cdots a-$ $1, a \cdots)$. From this we see that $X U_{h}=U_{f}$. If $g \preceq f$, then $g$ is of the form $(a|\cdots| \cdots a \cdots),(a-1|\cdots| \cdots a-1 \cdots),(a|a-1| \cdots a-1, a \cdots),(a-1|a| \cdots a-$ $1, a \cdots)$ or $(\cdots|a| \cdots a \cdots)$. So we have $Y^{2} g=0$, for $Y=E_{a-1}$.

In $(\mathrm{v})$ set $h=(a-1|a-1| \cdots a \cdots)$, while in (vi) set $h=(a-1|\cdots| \cdots a-$ $1, a \cdots)$. Here $X=F_{a-1}$. In either case we have $X U_{h}=U_{f}$ and if $g \preceq f$, then $Y^{2} g=0$, for $Y=E_{a-1}$.

\section{$\S 7.3 . \quad$ Proof of Theorem 7.2, II}

In this subsection, we consider the case when $\left|\Sigma_{f_{13}}\right|=0$ and $\left|\Sigma_{f_{23}}\right|=1$. Here we have the following possibilities.

(i) $f=(\cdots|a| \cdots a \cdots)$

(ii) $f=(\cdots|a| \cdots a-1, a \cdots)$

(iii) $f=(a-1|a| \cdots a \cdots)$.

In (i) we set $h=(\cdots|a-1| \cdots a \cdots)$, while in (ii) we set $h=(\cdots \mid a-$ $1 \mid \cdots a-1, a \cdots)$. In both cases $X=F_{a-1}$ and it is easy to see that in either case we have $X U_{h}=U_{f}$. In (i) if $g \preceq f$, then $g$ is of the form $(\cdots|a| \cdots a \cdots)$, $(a|\cdots| \cdots a \cdots),(\cdots|a-1| \cdots a-1 \cdots),(a-1|\cdots| \cdots a-1 \cdots),(a-1|a| \cdots a-$ $1, a \cdots)$ or $(a|a-1| \cdots a-1, a \cdots)$. Clearly $Y^{2} g=0$, for $Y=E_{a-1}$.

In (ii) if $g \preceq f$, then $g$ is of the form $(\cdots|a| \cdots a-1, a \cdots)$ or $(a|\cdots| \cdots a-$ $1, a \cdots)$. Also we have $Y^{2} g=0$. So in both cases the hypothesis of Theorem 5.3 is satisfied, and thus $U_{n}(f)=X U_{n}(h)$.

Finally for (iii) we consider first the case $f=(a-1|a| \cdots a \cdots)$, where $a-2$ is not contained in $\cdots$. We set $X=F_{a-2}$ and $h=(a-2|a| \cdots a \cdots)$. It is easy to check that $U_{f}=X U_{h}$. Next let $g=(a-2|a-1| \cdots a \cdots)$ and $X^{\prime}=F_{a-1}$. Again it is easy to see that $U_{h}=X^{\prime} U_{g}$, so that we have $U_{f}=X X^{\prime} U_{g}$. Now $g$ is typical and hence $U_{g}=K_{g}$. Thus we obtain

$$
U_{f}=X X^{\prime} K_{g}=K_{(a-1|a| \cdots a \cdots)}+q K_{(a-1|a-1| \cdots a-1 \cdots)}+q K_{(a-2|a-1| \cdots a-2 \cdots)}
$$

By Proposition $6.4 X X^{\prime} U_{n}(g)$ is isomorphic to $U_{n}(f)$. Now $X^{\prime} U_{n}(g)$ has a parabolic Verma flag of length two, and hence by Proposition 6.4 again, we see that $X^{\prime} U_{n}(g)=U_{n}(h)$. Thus we conclude that $X U_{n}(h)=U_{n}(f)$. 
We will use $x \sim y$ to denote the sequence of integers from $x$ to $y$. Suppose that $f=(a-1|a| \cdots,(a-k+1) \sim(a-2), a \cdots)$ and $a-k$ is not in $\cdots$, where $k \geq 3$. We consider the following sequence

$$
\begin{aligned}
& (a-1|a| \cdots,(a-k+1) \sim(a-2), a \cdots) \stackrel{E_{a-k}}{\leftarrow} \\
& (a-1|a| \cdots, a-k,(a-k+2) \sim(a-2), a \cdots) \stackrel{E_{a-k+1}}{\leftarrow} \\
& (a-1|a| \cdots a-k, a-k+1,(a-k+3) \sim(a-2), a \cdots) \stackrel{E_{a-k+2}}{\longleftarrow} \cdots \\
& \ldots \stackrel{E_{a-3}}{\leftarrow}(a-1|a| \cdots,(a-k) \sim(a-3), a \cdots)=g
\end{aligned}
$$

Lemma 7.4. Let $x, y$ and $a$ be distinct and $x, y>a$. Let $f=$ $(x|y| \cdots a \cdots y \cdots)$, where $\cdots$ denotes an expression with no $x$, $a$ and $a-1$. Let $h=(x|y| \cdots a-1 \cdots y \cdots)$ and $X=E_{a-1}$. Then $X U_{h}=U_{f}$.

Proof. Any $g \preceq h$ is of the form $(x|y| \cdots a-1 \cdots y \cdots),(y|x| \cdots a-$ $1 \cdots y \cdots),(x|a| \cdots a-1 \cdots a \cdots)$ or $(a|x| \cdots a-1 \cdots a \cdots)$.

Thus we have

$$
E_{a-k} E_{a-k+1} \cdots E_{a-4} E_{a-3} U_{g}=U_{f}
$$

Now $U_{g}=K_{(a-1|a| \cdots a \cdots)}+q K_{(a-1|a-1| \cdots a-1 \cdots)}+q K_{(a-2|a-1| \cdots a-2 \cdots)}$. A simple calculation shows that

$$
U_{f}=K_{(a-1|a| \cdots a \cdots)}+q K_{(a-1|a-1| \cdots a-1 \cdots)}+q K_{(a-k|a-1| \cdots a-k \cdots)} .
$$

Now Proposition 6.4 shows that $U_{n}(f)$ has a Verma flag consisting of parabolic Verma modules of these three highest weights. Now every $E_{a-k+i} \cdots$ $E_{a-4} E_{a-3} U_{g}$, for every $i>0$, contains three monomials, and thus $E_{a-k+i} \cdots$ $E_{a-4} E_{a-3} U_{n}(g)$ is a tilting module by Proposition 6.4. In particular $E_{a-k+1} \cdots$ $E_{a-4} E_{a-3} U_{n}(g)=U_{n}(h)$ and hence $E_{a-k} U_{n}(h)=U_{n}(f)$.

\section{§7.4. Formulas for canonical basis elements}

In this subsection, we provide a complete list of formulas for the canonical basis elements in $\mathcal{E}^{1,1 \mid n}$ (except the trivial case when $f$ is typical). They are computed using Procedure 7.1, and thus by Theorem 7.2 we find explicit Verma flag weights of the tilting modules in the category $\mathcal{O}_{1,1 \mid n}^{+}$as well.

Recall that we use $x \sim a$ to denote the sequence of integers from $x$ to $a$, and we shall use $\widehat{x} \sim a$ the sequence of integers from $x+1$ to $a$. We assume 
$c>a>b$.

Atypicality 2 :

(A1) $U_{(a|b| \cdots \widehat{x} \sim b \sim a \cdots)}=K_{(a|b| \cdots \widehat{x} \sim b \sim a \cdots)}+q K_{(b|a| \cdots \widehat{x} \sim b \sim a \cdots)}$

$$
\begin{aligned}
& +q K_{(a|x| \cdots x \sim \widehat{b} \sim a \cdots)}+q^{2} K_{(b|x| \cdots x \sim b \sim \widehat{a} \cdots)} \\
& +q^{2} K_{(x|a| \cdots x \sim \widehat{b} \sim a \cdots)}+q^{3} K_{(x|b| \cdots x \sim b \sim \widehat{a} \cdots)} .
\end{aligned}
$$

(A2) $U_{(a|b| \cdots \widehat{y} \sim b \cdots \widehat{x} \sim a \cdots)}=K_{(a|b| \cdots \widehat{y} \sim b \cdots \widehat{x} \sim a \cdots)}+q K_{(x|b| \cdots \widehat{y} \sim b \cdots x \sim \widehat{a} \cdots)}$

$$
\begin{aligned}
& +q K_{(a|y| \cdots y \sim \widehat{b} \cdots \widehat{x} \sim a \cdots)}+q K_{(b|a| \cdots \widehat{y} \sim b \cdots \widehat{x} \sim a \cdots)} \\
& +q^{2} K_{(b|x| \cdots \widehat{y} \sim b \cdots x \sim \widehat{a} \cdots)}+q^{2} K_{(y|a| \cdots y \sim \widehat{b} \cdots \widehat{x} \sim a \cdots)} \\
& +q^{2} K_{(x|y| \cdots y \sim \widehat{b} \cdots x \sim \widehat{a} \cdots)}+q^{3} K_{(y|x| \cdots y \sim \widehat{b} \cdots x \sim \widehat{a} \cdots)} .
\end{aligned}
$$

(A3) $U_{(b|a| \cdots \widehat{y} \sim \widehat{x} \sim b \sim a \cdots)}=K_{(b|a| \cdots \widehat{y} \sim \widehat{x} \sim b \sim a \cdots)}+q K_{(x|a| \cdots \widehat{y} \sim x \sim \widehat{b} \sim a \cdots)}$

$$
\begin{aligned}
& +q K_{(b|x| \cdots \widehat{y} \sim x \sim b \sim \widehat{a} \cdots)}+q K_{(y|b| \cdots y \sim \widehat{x} \sim b \sim \widehat{a} \cdots)} \\
& +q^{2} K_{(x|b| \cdots \widehat{y} \sim x \sim b \sim \widehat{a} \cdots)}+q^{2} K_{(y|x| \cdots y \sim x \sim \widehat{b} \sim \widehat{a} \cdots)} .
\end{aligned}
$$

(A4) $U_{(b|a| \cdots \widehat{y} \sim b \cdots \widehat{x} \sim a \cdots)}=K_{(b|a| \cdots \widehat{y} \sim b \cdots \widehat{x} \sim a \cdots)}+q K_{(y|a| \cdots y \sim \widehat{b} \cdots \widehat{x} \sim a \cdots)}$

$$
+q K_{(b|x| \cdots \widehat{y} \sim b \cdots x \sim \widehat{a} \cdots)}+q^{2} K_{(y|x| \cdots y \sim \widehat{b} \cdots x \sim \widehat{a} \cdots)} .
$$

Atypicality $1 \quad(b<x<a$ is assumed below $)$ :

(B1) $U_{(a|c| \cdots \widehat{x} \sim a \cdots)}=K_{(a|c| \cdots \widehat{x} \sim a \cdots)}+q K_{(x|c| \cdots x \sim \widehat{a} \cdots)}$.

(B2) $U_{(a|b| \cdots \widehat{x} \sim a \cdots)}=K_{(a|b| \cdots \widehat{x} \sim a \cdots)}+q K_{(b|a| \cdots \widehat{x} \sim a \cdots)}+q K_{(x|b| \cdots x \sim \widehat{a} \cdots)}$

$$
+q^{2} K_{(b|x| \cdots x \sim \widehat{a} \cdots)} \text {. }
$$

(B3) $U_{(a|x| \cdots \widehat{x} \sim a \cdots)}=K_{(a|x| \cdots \widehat{x} \sim a \cdots)}+q K_{(x|a| \cdots \widehat{x} \sim a \cdots)}+q^{2} K_{(x|x| \cdots x \sim \widehat{a} \cdots)}$.

(B4) $U_{(c|a| \cdots \widehat{x} \sim a \cdots)}=K_{(c|a| \cdots \widehat{x} \sim a \cdots)}+q K_{(a|c| \cdots \widehat{x} \sim a \cdots)}+q K_{(c|x| \cdots x \sim \widehat{a} \cdots)}$

$$
+q^{2} K_{(x|c| \cdots x \sim \widehat{a} \cdots)} \text {. }
$$

(B5) $U_{(b|a| \cdots \widehat{x} \sim a \cdots)}=K_{(b|a| \cdots \widehat{x} \sim a \cdots)}+q K_{(b|x| \cdots x \sim \widehat{a} \cdots)}$.

(B6) $U_{(x|a| \cdots \widehat{y} \sim \widehat{x} \sim a \cdots)}=K_{(x|a| \cdots \widehat{y} \sim \widehat{x} \sim a \cdots)}+q K_{(x|x| \cdots \widehat{y} \sim x \sim \widehat{a} \cdots)}$

$$
+q K_{(y|x| \cdots y \sim \widehat{x} \sim \widehat{a} \cdots)} .
$$

(S) $U_{(a|a| \cdots \widehat{x} \sim a \cdots)}=K_{(a|a| \cdots \widehat{x} \sim a \cdots)}+q K_{(a|x| \cdots x \sim \widehat{a} \cdots)}+q^{2} K_{(x|a| \cdots x \sim \widehat{a} \cdots)}$.

(This last weight is special in the sense that it has three identical values.)

\section{$\S 7.5$. Super duality: a weak version}

The following weak version of Conjecture 4.18 holds in the case $\mathbf{m}=(1,1)$. 
Theorem 7.5. The categories $\mathcal{O}_{1,1 \mid \infty}^{+}$and $\mathcal{O}_{(1,1)+\infty}^{+}$admit isomorphic Kazhdan-Lusztig theories. In particular, for $f, g \in \mathbb{Z}_{+}^{(1,1)+\infty}$ we have

$$
\begin{aligned}
& (\mathcal{U}(f): \mathcal{K}(g))=\left(U\left(f^{\natural}\right): K\left(g^{\natural}\right)\right), \\
& {[\mathcal{K}(f): \mathcal{L}(g)]=\left[K\left(f^{\natural}\right): L\left(g^{\natural}\right)\right] .}
\end{aligned}
$$

Proof. In light of Theorem 7.2 (iii), Theorem 3.14 and Corollary 2.6 we see that

$$
u_{g f}(1)=u_{g^{(n)} f^{(n)}}(1)=\left(U_{n}\left(f^{(n)}\right): K_{n}\left(g^{(n)}\right)\right)=(U(f): K(g)), \quad n \gg 0 .
$$

By Theorem 4.16, Theorem 4.14 and Proposition 4.4 we have for $f^{\prime}, g^{\prime} \in$ $\mathbb{Z}_{+}^{(1,1)+\infty}$

$$
\mathfrak{u}_{g^{\prime}, f^{\prime}}(1)=\mathfrak{u}_{g^{\prime(n)}, f^{\prime(n)}}(1)=\left(\mathcal{U}_{n}\left(f^{\prime(n)}\right): \mathcal{K}_{n}\left(g^{\prime(n)}\right)\right)=\left(\mathcal{U}\left(f^{\prime}\right): \mathcal{K}\left(g^{\prime}\right)\right), \quad n \gg 0 .
$$

Now the first identity in the theorem follows by Theorem 4.7.

The second identity in the theorem follows by Remarks 2.3 and 4.3, Theorem 4.15 and (3.2), together with the corresponding compatibility of truncation functors on irreducible representations and truncation maps on dual canonical basis elements.

\section{$\S 8$. The Category $\mathcal{O}_{m, 1 \mid 1}^{+}$of $\mathfrak{g r}(m+1 \mid 1)$-Modules}

In this section, we analyze completely the case for $\mathbf{m}=(m, 1)$ and $n=$ 1. We find explicit formulas for canonical basis in $\mathcal{E}^{m, 1 \mid 1}$, and establish the parabolic BKL Conjecture 3.10 for the category $\mathcal{O}_{m, 1 \mid 1}^{+}$.

\section{§8.1. A procedure}

Denote $\Sigma_{f_{13}}=(i \mid 1)$ if there exists $i<-1$ with $f(i)=f(1)$, and otherwise set $\Sigma_{f_{13}}=\emptyset$. Also denote $\Sigma_{f_{23}}=(-1 \mid 1)$ if $f(-1)=f(1)$, and otherwise set $\Sigma_{f_{23}}=\emptyset$. If $\Sigma_{f_{13}} \cup \Sigma_{f_{23}}=\emptyset$, then $\# f=0$.

Procedure 8.1. Let $f \in \mathbb{Z}_{+}^{m, 1 \mid 1}$ be such that $\Sigma_{f_{13}} \cup \Sigma_{f_{23}} \neq \emptyset$.

Step 1 If $\Sigma_{f_{13}}=\emptyset$, go to Step 5 . Otherwise let $\Sigma_{f_{13}}=(i \mid 1)$ and go to Step 2 .

Step 2 If $i<-1$ and $f(i+1)=f(i)-1$, replace $i$ by $i+1$ and repeat Step 2 .

Otherwise go to Step 3. 
Step 3 If $f(i)=f(-1)$, go to Step 4. Otherwise we set $h(i)=f(i)-1$ and $h(s)=f(s)$, for $s \neq i$. Let $X=F_{f(i)-1}$. Stop.

Step 4 Set $h(i)=h(-1)=f(i)-1$, and $h(s)=f(s)$, for $s \neq i,-1$. Let $X=F_{f(i)-1}^{(2)}$. Stop.

Step 5 We have $f(-1)=f(1)$. If there exists $i<-1$ such that $f(i)=f(-1)-$ 1, go to Step 2. Otherwise set $h(-1)=f(-1)-1$, and $h(s)=f(s)$, for $s \neq-1$. Let $X=F_{f(-1)-1}$. Stop.

\section{§8.2. Formulas for canonical basis}

We will leave the straightforward verification of the following to the reader.

Proposition 8.2. $\quad$ Let $f$ be such that $\Sigma_{f_{13}} \cup \Sigma_{f_{23}} \neq \emptyset$. Let $X$ and $h$ be as defined in Procedure 8.1. Then we have $X U_{h}=U_{f}$.

Repeated application of Procedure 8.1 will produce an element $g$ with $\# g=0$. By Proposition 6.5 we have $U_{g}=U_{g_{12}} \otimes w_{g(1)}$. Thus the above procedure computes all canonical basis elements in $\mathcal{E}^{m, 1 \mid 1}$. Below we present a complete list of formulas for the canonical basis elements (except the really simple case when $f$ is typical). We caution that some cases will be missing if $m$ is too small.

Atypical cases:

$$
\begin{aligned}
& \text { (C1) } U_{(\cdots a \sim b \sim \widehat{x} \cdots|b| a)}=K_{(\cdots a \sim b \sim \widehat{x} \cdots|b| a)}+q K_{(\cdots \widehat{a} \sim b \sim x \cdots|b| x)}, \quad a>b>x . \\
& \text { (C2) } U_{(\cdots a \sim \widehat{x} \cdots|x| a)}=K_{(\cdots a \sim \widehat{x} \cdots|x| a)}+q K_{(\cdots a \sim \widehat{x+1}, x \cdots|x+1| a)} \\
& +q K_{(\cdots \widehat{a} \sim x \cdots|x+1| x+1)}+q^{2} K_{(\cdots \widehat{a} \sim x \cdots|x| x), \quad a-1>x .} \\
& \text { (C3) } U_{(\cdots a, \widehat{a-1} \cdots|a-1| a)}=K_{(\cdots a, \widehat{a-1} \cdots|a-1| a)}+q K_{(\cdots \widehat{a}, a-1 \cdots|a| a)} \\
& +q^{2} K_{(\cdots \widehat{a}, a-1 \cdots|a-1| a-1)} . \\
& \text { (C4) } U_{(\cdots c, \widehat{a} \sim \widehat{x} \cdots|a| a)}=K_{(\cdots c, \widehat{a} \sim \widehat{x} \cdots|a| a)}+q K_{(\cdots \widehat{c}, a \sim \widehat{x} \cdots|c| a)} \\
& +q K_{(\cdots c, \widehat{a} \sim \widehat{x} \cdots|a-1| a-1)}+q K_{(\cdots c, \widehat{a-1} \sim x \cdots|a-1| x)} \\
& +q^{2} K_{(\cdots \widehat{c}, a-1 \sim x \cdots|c| x), \quad a-1>x .} \\
& \text { (C5) } U_{(\widehat{a} \sim \widehat{x} \cdots|a| a)}=K_{(\widehat{a} \sim \widehat{x} \cdots|a| a)}+q K_{(\widehat{a} \sim \widehat{x} \cdots|a-1| a-1)} \\
& +q K_{(\widehat{a}, \widehat{a-1} \sim x \cdots|a-1| x)}, a-1>x . \\
& \text { (C6) } U_{(\widehat{a}, \widehat{a-1} \cdots|a| a)}=K_{(\widehat{a}, \widehat{a-1} \cdots|a| a)}+q K_{(\widehat{a}, \widehat{a-1} \cdots|a-1| a-1)} \text {. }
\end{aligned}
$$


$(C 7)$

$$
\begin{gathered}
\text { (C7) } U_{(\cdots c, \widehat{a}, \widehat{a-1} \cdots|a| a)}=K_{(\cdots c, \widehat{a}, \widehat{a-1} \cdots|a| a)}+q K_{(\cdots \widehat{c}, a, \widehat{a-1} \cdots|c| a)} \\
+q K_{(\cdots c, \widehat{a}, \widehat{a-1} \cdots|a-1| a-1)}+q^{2} K_{(\cdots \widehat{c}, a-1 \cdots|c| a-1)} . \\
(C 8) \quad U_{(\cdots d, \widehat{c} \cdots a \sim \widehat{x} \cdots|c| a)}=K_{(\cdots d, \widehat{c} \cdots a \sim \widehat{x} \cdots|c| a)}+q K_{(\cdots d, \widehat{c} \cdots \widehat{a} \sim x \cdots|c| x)} \\
+q K_{(\cdots \widehat{d}, c \cdots a \sim \widehat{x} \cdots|d| a)}+q^{2} K_{(\cdots \widehat{d}, c \cdots \widehat{a} \sim x \cdots|d| x)}, \quad d>c>a . \\
(C 9) \quad U_{(\cdots a \sim \widehat{x} \cdots e, \widehat{b} \cdots|b| a)}=K_{(\cdots a \sim \widehat{x} \cdots e, \widehat{b} \cdots|b| a)}+q K_{(\cdots \widehat{a} \sim x \cdots e, \widehat{b} \cdots|b| x)} \\
+q K_{(\cdots a \sim \widehat{x} \cdots \widehat{e}, b \cdots|e| a)}+q^{2} K_{(\cdots \widehat{a} \sim x \cdots \widehat{e}, b \cdots|e| x)}, \quad x>e>b .
\end{gathered}
$$

(T1) $U_{(\cdots a \sim \widehat{x} \cdots|a| a)}=K_{(\cdots a \sim \widehat{x} \cdots|a| a)}+q K_{(\cdots a \sim \widehat{x} \cdots|a-1| a-1)}$

$$
+q K_{(\cdots a, \widehat{a-1} \sim x \cdots|a-1| x)}+q^{2} K_{(\cdots \widehat{a} \sim x \cdots|a| x), \quad a-1>x .}
$$

$$
\begin{aligned}
U_{(\cdots a, \widehat{a-1} \cdots|a| a)} & =K_{(\cdots a, \widehat{a-1} \cdots|a| a)}+q K_{(\cdots a, \widehat{a-1} \cdots|a-1| a-1)} \\
& +q^{2} K_{(\cdots \widehat{a}, a-1 \cdots|a| a-1)} .
\end{aligned}
$$

(In the cases (T1, T2) the weights have three identical values.)

The case of (C8) (respectively (C9)), when no such $d$ (respectively e) exists, is obtained by dropping the last two terms.

\section{$\S 8.3$. Structure of tilting modules in $\mathcal{O}_{m, 1 \mid 1}^{+}$}

We shall denote the tilting modules in $\mathcal{O}_{m, 1 \mid 1}^{+}$by $U(f)$ et cetera.

Theorem 8.3. For any $f \in \mathbb{Z}_{+}^{m, 1 \mid 1}$ we have $i([U(f)])=U_{f}(1)$.

Proof. For typical $f$, this follows from Proposition 6.5. So let us now assume that $f$ is atypical.

Each canonical basis element $U_{f}$ in Subsection 8.2 is obtained by applying a sequence of Chevalley generators dictated by Procedure 8.1 to a canonical basis element of typical weight. Applying the same sequence of translation functors gives us a sum of tilting modules, denoted by $M(f)$, whose Verma flag weights are identical to those for the monomials in $U_{f}$, by Lemma 6.2 and Theorem 3.9. It follows by Proposition 8.2 that $i([M(f)])=U_{f}(1)$. So it remains to show that $M(f)=U(f)$. Noting that $U(f)$ is a summand of $M(f)$, it suffices to prove that $M(f)$ is indecomposable. We argue case by case using Proposition 6.1 and Proposition 6.4 as follows.

The indecomposability of $M(f)$ follows from Proposition 6.4 if the number of monomials is at most three. So it remains to check the cases of (T1), (C2), $(\mathrm{C} 4),(\mathrm{C} 7)$ and $(\mathrm{C} 8)$ and $(\mathrm{C} 9)$ (in the last cases we only need to consider them when they have four terms). 
For $f$ of the form in (T1), the Verma modules with the first two weights among four weights in (T1) must lie in the same tilting module by Proposition 6.1 (ii). Now $M(f)$ is a direct sum of at most two tilting modules, by Lemma 6.3 . If $M(f)$ were a direct sum of two tilting modules, it has to be $U(f) \oplus U\left(f^{3}\right)$ where $f^{3}=(\cdots a, \widehat{a-1} \sim x \cdots|a-1| x)$ and $f^{4}=$ $(\cdots \widehat{a} \sim x \cdots|a| x)$ are the third and fourth weights in $(\mathrm{T} 1)$. Note that $\left(U\left(f^{3}\right)\right.$ : $\left.K\left(f^{3}\right)\right)=\left(U\left(f^{3}\right): K\left(f^{4}\right)\right)=1$ and that the cosocle of $U\left(f^{3}\right)$ is $L\left(f^{4}\right)$. However, $L\left(f^{4}\right)$ cannot be the socle of $K\left(f^{3}\right)$. For consider the embedding of $\mathfrak{g l}(m+1) \otimes \mathfrak{g l}(1)$-modules $K^{12}(\cdots a, \widehat{a-1} \sim x \cdots \mid a-1) \otimes L^{3}(x) \supsetneq K^{12}(\cdots \widehat{a}, a-$ $1 \sim x \cdots \mid a) \otimes L^{3}(x)$, which we may regard as an embedding of $\mathfrak{p}$-modules. Inducing to $\mathfrak{g l}(m+1 \mid 1)$ we get an embedding $K\left(f^{3}\right) \supsetneq K\left(f^{4}\right)$. But $K\left(f^{4}\right)$ is not irreducible, and its socle is not $L\left(f^{4}\right)$. This implies that $U\left(f^{3}\right)$ cannot have isomorphic socle and cosocle and hence is not $\tau$-self-dual, contradicting Corollary 3.8 .

Next consider a weight $f$ of the form in (C2). Since the Verma modules of the first two weights in (C2) belong to the same tilting module by Proposition 6.1 (i), we have by Lemma 6.3 that $M(f)=U(f)$ or $M(f)=U(f) \oplus U\left(f^{3}\right)$, where $f^{3}$ is the third weight in $(\mathrm{C} 2)$. But the second possibility cannot occur since $f^{3}$ is of the form (T1) and $U\left(f^{3}\right)$ has Verma flag length four by the previous paragraph.

For $f$ of the form in (C4), the second and the third weights are not comparable under the super Bruhat ordering. Hence using Proposition 6.1 the first three terms lie in the tilting module $U(f)$. By Lemma 6.3, $M(f)$ has to be indecomposable, and thus equal to $U(f)$.

The same argument for (C4) is applicable to (C7).

Finally, the two cases of (C8) and (C9) in the case when we have four terms can be verified using Proposition 6.1 (i) and the socle-cosocle argument.

Remark 8.4. In light of the above theorem, the formulas for canonical basis in Subsection 8.2 provide explicit information on the weights of a Verma flag of any tilting module in $\mathcal{O}_{m, 1 \mid 1}^{+}$.

Corollary 8.5. Let $f$ be such that $\Sigma_{f_{13}} \cup \Sigma_{f_{23}} \neq \emptyset$. Let $X$ and $h$ be defined as in Procedure 8.1. Then we have $U(f)=X U(h)$.

\section{$\S 9$. The Category of $\mathfrak{g l}(2 \mid 1)$-Modules}

In this section we work out explicitly the Verma flag structures for tilting modules, projective modules, and the composition series of Verma modules in 
the category $\mathcal{O}_{2 \mid 1}^{+}$. The results here can be generalized to the category $\mathcal{O}_{m+1 \mid 1}^{+}$in Section 8 readily and to the category $\mathcal{O}_{1+1 \mid n}^{+}$in Section 7 with more complicated notations.

\section{§9.1. The main tools}

Denote by $P(\lambda)$ the projective cover of $L(\lambda)$. By abuse of notations, we shall also write $P\left(f_{\lambda}\right)=P(\lambda)$. Recall the BGG reciprocity for projective modules:

$$
\left(P\left(f_{\lambda}\right): K\left(f_{\mu}\right)\right)=\left[K\left(f_{\mu}\right): L\left(f_{\lambda}\right)\right]
$$

By $[\mathrm{Br} 2,(7.4)]$,

$$
\left(U\left(f_{\lambda}\right): K\left(f_{\mu}\right)\right)=\left[K\left(-f_{\mu}\right): L\left(-f_{\lambda}\right)\right] .
$$

In the following diagrams, $\bar{i}$ (for $i>0$ ) denotes $-i$, and the weights are described using elements in $\mathbb{Z}^{2 \mid 1}$ via the bijection $X_{2 \mid 1} \cong \mathbb{Z}^{2 \mid 1}, \lambda \mapsto f_{\lambda}$. We will be only concerned about the block $\mathfrak{B}$ of $K(00 \mid 0)$ in the category $\mathcal{O}_{2 \mid 1}^{+}$. Any block of atypicality 1 in the category $\mathcal{O}_{2 \mid 1}^{+}$is isomorphic to $\mathfrak{B}$. A block of atypicality 0 is very simple and will be omitted.

\section{$\S 9.2$. The poset of weights in the block $\mathfrak{B}$}

The poset of $\rho$-shifted weights in $\mathbb{Z}^{2 \mid 1}$ for the block $\mathfrak{B}$ is listed in the following diagram. Our convention is that arrows point to lower weights in the super Bruhat ordering. 


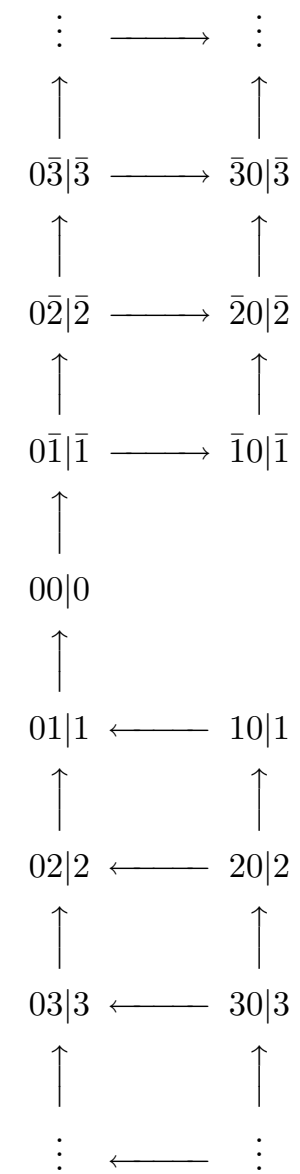

$\S 9.3$. The Verma flag structures of tilting modules in $\mathfrak{B}$

Based on Theorem 7.2 (with $n=1$ ) or Theorem 8.3 (with $m=1$ ) and the explicit formulas for canonical basis in Subsection 8.2, we list the weights of the Verma modules (each with multiplicity 1) which appear in a Verma flag of a tilting module $U(f)$ in the block $\mathfrak{B}$ as follows. Recall from Theorem 7.2 that every such $U(f)$ has a simple cosocle. 


$$
\begin{aligned}
& 0 \overline{i+1}|\overline{i+1} \longrightarrow \overline{i+1} 0| \overline{i+1} \\
& U(0 \bar{i} \mid \bar{i}) \approx \uparrow_{0 \bar{i} \mid \bar{i}} \longrightarrow \underset{\bar{i} 0 \mid \bar{i}}{\uparrow} \quad(i \geq 1) \\
& \overline{i+1} 0 \mid \overline{i+1} \\
& U(\bar{i} 0 \mid \bar{i}) \approx \uparrow_{\bar{i} 0 \mid \bar{i}} \quad(i \geq 1) \\
& U(00 \mid 0) \approx \uparrow_{00 \mid 0}^{0 \overline{1}|\overline{1} \longrightarrow \overline{1} 0| \overline{1} \quad} \quad 00 \mid 0 \longrightarrow \overline{10} \longrightarrow \overline{1} \\
& 00 \mid 0 \\
& U(10 \mid 1) \approx \uparrow \\
& 01|1 \longleftarrow 10| 1 \\
& 0, j-1 \mid j-1 \\
& U(0 j \mid j) \approx \quad \uparrow \quad(j \geq 2) \\
& 0 j \mid j \\
& 0, j-1|j-1 \longleftarrow j-1,0| j-1 \\
& U(j 0 \mid j) \approx \underset{0 j \mid j}{ } \longleftarrow \underset{j 0 \mid j}{\uparrow} \quad(j \geq 2)
\end{aligned}
$$

\section{§9.4. The composition series of Verma modules in $\mathfrak{B}$}

The weights of the composition factors of a Verma module $K(f)$ in the block $\mathfrak{B}$ are listed as follows. The calculation is based on (9.2) and the Verma flag structure of tilting modules in Subsection 9.3. 


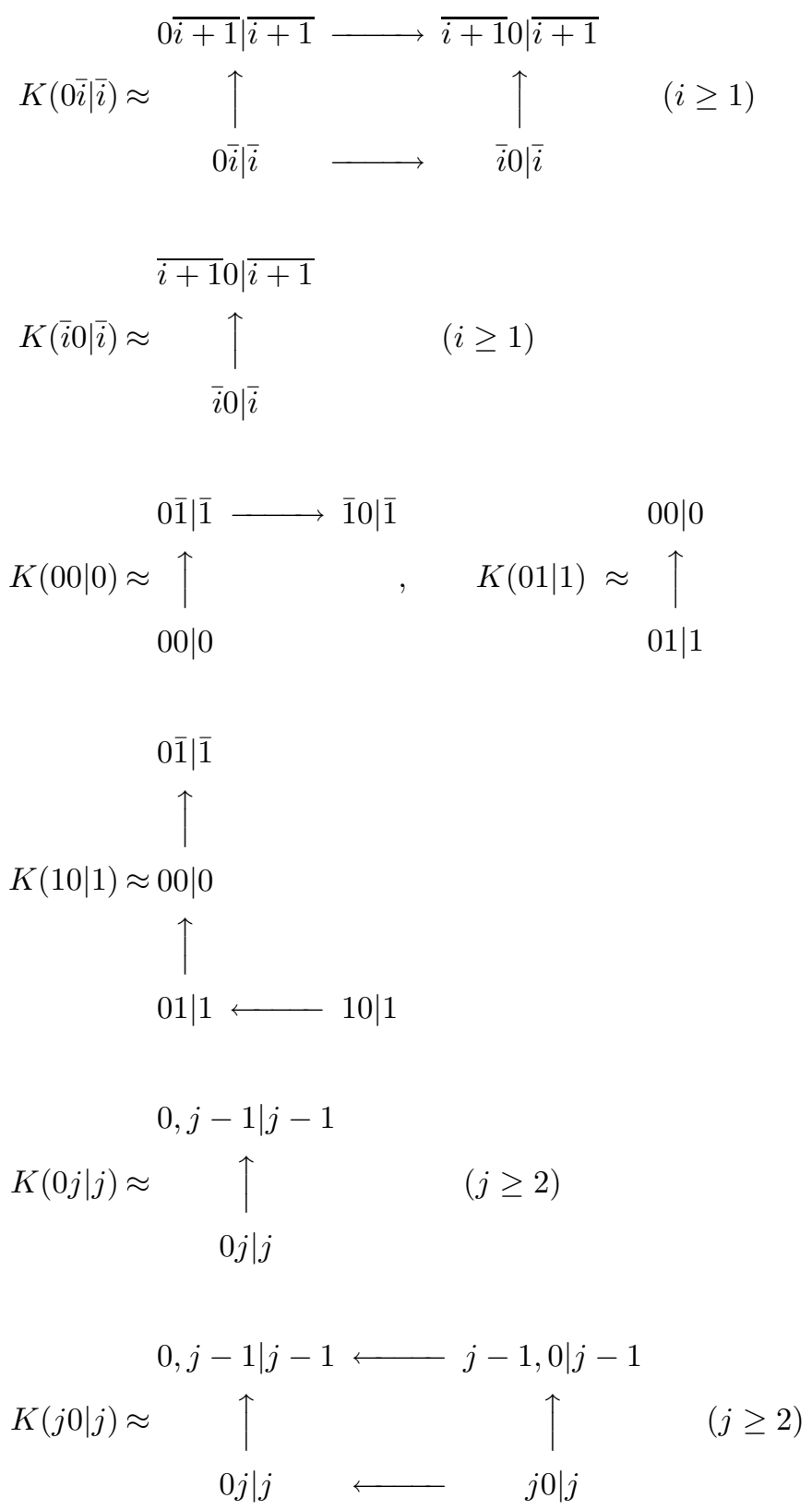




\section{§9.5. The Verma flag structures of projective modules in $\mathfrak{B}$}

The weights of the Verma modules (each with multiplicity 1) which appear in a Verma flag of a projective module in the block $\mathfrak{B}$ are listed as follows. The calculation is based on (9.1) and Subsection 9.4.

$$
\begin{aligned}
& 0 \bar{i}|\bar{i} \quad \longrightarrow \quad \bar{i} 0| \bar{i}
\end{aligned}
$$

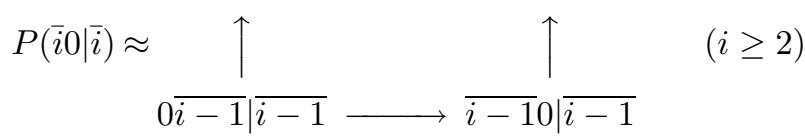

$$
\begin{aligned}
& 0 \bar{i} \mid \bar{i} \\
& P(0 \bar{i} \mid \bar{i}) \approx \uparrow_{0 \overline{i-1} \mid \overline{i-1}}^{\uparrow} \quad(i \geq 2) \\
& 0 \overline{1}|\overline{1} \longrightarrow \overline{1} 0| \overline{1} \quad 0 \overline{1} \mid \overline{1} \\
& P(\overline{1} 0 \mid \overline{1}) \approx \uparrow \quad, \quad P(0 \overline{1} \mid \overline{1}) \approx \uparrow \\
& 00|0 \quad 00| 0 \longleftarrow 10 \mid 1 \\
& 00 \mid 0 \\
& P(00 \mid 0) \approx \uparrow \\
& 01|1 \longleftarrow 10| 1 \\
& P(j 0 \mid j) \approx \prod_{j+1,0 \mid j+1}^{j 0 \mid j} \quad(j \geq 1) \\
& P(0 j \mid j) \approx \underset{\uparrow}{0 j \mid j} \longleftarrow \underset{j}{j 0 \mid j} \prod_{j+1,0 \mid j+1} \quad(j \geq 1)
\end{aligned}
$$




\section{§9.6. The projective tilting modules in $\mathfrak{B}$}

By Theorem 7.2 for $n=1$, the tilting module $U(0 \overline{i-1} \mid \overline{i-1})$ in the block $\mathfrak{B}$ has a simple cosocle $L(\bar{i} 0 \mid \bar{i})$ for $i \geq 1$. Thus the nontrivial $\mathfrak{g l}(2 \mid 1)$-module homomorphism $\pi_{-i}: P(\bar{i} 0 \mid \bar{i}) \longrightarrow U(0 \overline{i-1} \mid \overline{i-1})$ has to be surjective. By observation from the previous diagrams, $U(0 \overline{i-1} \mid \overline{i-1})$ and $P(\bar{i} 0 \mid \bar{i})$ have the same Verma flag multiplicity and thus the same composition series. It follows that $\pi_{-i}$ is indeed an isomorphism.

Similarly, there is a $\mathfrak{g l}(2 \mid 1)$-module isomorphism $\pi_{i}: P(0 i \mid i) \longrightarrow U(i+$ $1,0 \mid i+1)$ for $i \geq 0$. Again by observation from the diagrams, the remaining tilting modules are not projective.

The above discussion can be summarized in the following.

Proposition 9.1. The projective tilting modules in the category $\mathrm{O}_{2 \mid 1}^{+}$ consist of $U(i 0 \mid i)$ for $i \geq 0$ and $U(0 j \mid j)$ for $j<0$.

\section{Acknowledgments}

S-J.C. is partially supported by an NSC grant of the R.O.C. and an Academia Sinica Investigator grant. He also thanks the Department of Mathematics, University of Virginia, for hospitality and support. W.W. is partially supported by NSF. We are grateful to Jon Brundan for his influential ideas and several stimulating discussions. We also thank R.B. Zhang for his participation at an early stage of this work and in [CWZ]. Notation: $\mathbb{N}=\{0,1,2, \cdots\}$.

\section{References}

[AS] T. Arakawa and T. Suzuki, Duality between $\mathfrak{s l}_{n}(\mathbb{C})$ and the degenerate affine Hecke algebra, J. Algebra 209 (1998), no. 1, 288-304.

[BB] A. Bẹlinson and J. Bernstein, Localisation de $g$-modules, C. R. Acad. Sci. Paris Sér. I Math. 292 (1981), no. 1, 15-18.

[BGS] A. Beilinson, V. Ginzburg and W. Soergel, Koszul duality patterns in representation theory, J. Amer. Math. Soc. 9 (1996), no. 2, 473-527.

[Br1] J. Brundan, Kazhdan-Lusztig polynomials and character formulae for the Lie superalgebra $\mathfrak{g l}(m \mid n)$, J. Amer. Math. Soc. 16 (2003), no. 1, 185-231 (electronic).

[Br2] _ Tilting modules for Lie superalgebras, Comm. Algebra 32 (2004), no. 6 , $2251-2268$.

[Br3] Kazhdan-Lusztig polynomials and character formulae for the Lie superalgebra $\mathfrak{q}(n)$, Adv. Math. 182 (2004), no. 1, 28-77.

[BKl] J. Brundan and A. Kleshchev, Representations of shifted Yangians and finite $W$ algebras, Memoirs AMS (to appear), math.RT/0508003.

[BK] J.-L. Brylinski and M. Kashiwara, Kazhdan-Lusztig conjecture and holonomic systems, Invent. Math. 64 (1981), no. 3, 387-410.

[CR] J. Chuang and R. Rouquier, Derived equivalences for symmetric groups and $\mathfrak{s l}_{2}-$ categorification, Ann. of Math. (2) 167 (2008), no. 1, 245-298. 
[CWZ] S.-J. Cheng, W. Wang and R. B. Zhang, Super duality and Kazhdan-Lusztig polynomials, Trans. Amer. Math. Soc. 360 (2008), 5883-5924.

[CWZ2] - A Fock space approach to representation theory of $\operatorname{osp}(2 \mid 2 n)$, Transform. Groups 12 (2007), no. 2, 209-225.

[CZ] S.-J. Cheng and R. B. Zhang, Analogue of Kostant's u-cohomology formula for the general linear superalgebra, Int. Math. Res. Not. 2004, no. 1, 31-53.

[CoI $]$ D. H. Collingwood and R. S. Irving, A decomposition theorem for certain self-dual modules in the category $\mathcal{O}$, Duke Math. J. 58 (1989), no. 1, 89-102.

[Deo] V. V. Deodhar, On some geometric aspects of Bruhat orderings. II. The parabolic analogue of Kazhdan-Lusztig polynomials, J. Algebra 111 (1987), no. 2, 483-506.

[Don] S. Donkin, On tilting modules for algebraic groups, Math. Z. 212 (1993), no. 1, 39-60.

[FKK] I. B. Frenkel, M. G. Khovanov and A. A. Kirillov, Jr., Kazhdan-Lusztig polynomials and canonical basis, Transform. Groups 3 (1998), no. 4, 321-336.

[FKS] I. Frenkel, M. Khovanov and C. Stroppel, A categorification of finite-dimensional irreducible representations of quantum $\mathfrak{s l}_{2}$ and their tensor products, Selecta Math. (N.S.) 12 (2006), no. 3-4, 379-431.

[Jan] J. C. Jantzen, Representations of algebraic groups, Second edition, Amer. Math. Soc., Providence, RI, 2003.

[Jim] M. Jimbo, A $q$-analogue of $U(\mathfrak{g l}(N+1))$, Hecke algebra, and the Yang-Baxter equation, Lett. Math. Phys. 11 (1986), no. 3, 247-252.

[K1] V. G. Kac, Lie superalgebras, Advances in Math. 26 (1977), no. 1, 8-96.

[K2] Representations of classical Lie superalgebras, in Differential geometrical methods in mathematical physics, II (Proc. Conf., Univ. Bonn, Bonn, 1977), 597626, Lecture Notes in Math., 676, Springer, Berlin, 1978.

[Kas] M. Kashiwara, On crystal bases of the $Q$-analogue of universal enveloping algebras, Duke Math. J. 63 (1991), no. 2, 465-516.

[KL1] D. Kazhdan and G. Lusztig, Representations of Coxeter groups and Hecke algebras, Invent. Math. 53 (1979), no. 2, 165-184.

[KL2] _ Schubert varieties and Poincare duality, in Geometry of the Laplace operator (Proc. Sympos. Pure Math., Univ. Hawaii, Honolulu, Hawaii, 1979), 185-203, Proc. Sympos. Pure Math., XXXVI, Amer. Math. Soc., Providence, R.I, 1980.

[KMS] M. Kashiwara, T. Miwa and E. Stern, Decomposition of $q$-deformed Fock spaces, Selecta Math. (N.S.) 1 (1995), no. 4, 787-805.

[Lu1] G. Lusztig, Canonical bases arising from quantized enveloping algebras, J. Amer. Math. Soc. 3 (1990), no. 2, 447-498.

[Lu2] _ Introduction to quantum groups, Progr. Math., 110, Birkhäuser Boston, Boston, MA, 1993.

[Lu3] Private communication, Email to W. Wang, September 10, 2007.

[Se] V. Serganova, Kazhdan-Lusztig polynomials and character formula for the Lie superalgebra $\mathfrak{g l}(m \mid n)$, Selecta Math. (N.S.) 2 (1996), no. 4, 607-651.

[So1] W. Soergel, Kazhdan-Lusztig polynomials and a combinatoric[s] for tilting modules, Represent. Theory 1 (1997), 83-114 (electronic).

[So2] _ Character formulas for tilting modules over Kac-Moody algebras, Represent. Theory 2 (1998), 432-448 (electronic). 